\title{
AN ELECTRO-OPTICAL SIMULATION METHODOLOGY FOR THE ANALYSIS OF SINGLE-EVENT RADIATION EFFECTS IN PHOTONIC DEVICES
}

\author{
By \\ Ryan Boggs
}

T. Daniel Loveless

UC Foundation Assistant Professor of Electrical Engineering (Chair)

Donald R. Reising

Assistant Professor of Electrical Engineering

(Committee Member)

Kidambi Sreenivas

Associate Professor of Mechanical Engineering

(Committee Member) 


\title{
AN ELECTRO-OPTICAL SIMULATION METHODOLOGY FOR THE ANALYSIS OF SINGLE-EVENT RADIATION EFFECTS IN PHOTONIC DEVICES
}

\author{
By \\ Ryan Boggs \\ A Thesis Submitted to the Faculty of the University of \\ Tennessee at Chattanooga in Partial \\ Fulfillment of the Requirements of the Degree \\ of Master of Science: Engineering
}

The University of Tennessee at Chattanooga

Chattanooga, Tennessee

August 2019 


\begin{abstract}
Photonic integrated circuits (PICs) are devices that transmit and perform operations on optical signals. PICs offer significant benefits compared to conventional electronics, including increased data transmission rates. Despite the desire to leverage these benefits for space systems increasing in recent years, the study of radiation effects in PICs is still limited, especially the transient radiation effects known as single-event effects (SEEs). A roadblock in advancing the understanding of SEEs in PICs is the inability to co-simulate radiation effects in both the optical and electrical domains. Establishing a means of simulating SEEs in PICs would aide in: (1) modeling SEEs in various devices, (2) determining specific mechanisms by which SEEs upset devices, and (3) discovering methods for improving the radiation hardness of PICs. This work introduces a methodology for the simulation of SEEs in PICs that couples optical and electrical domain simulations that are required to fully characterize SEEs in PICs.
\end{abstract}




\section{DEDICATION}

I dedicate this work to my mom and dad, who have done everything possible to support me in developing my future and encourage me to follow my passion in engineering. 


\section{ACKNOWLEDGEMENTS}

I would first like to thank the Defense Threat Reduction Agency for their support of this work. I would also like to thank Dr. Loveless for his guidance and support throughout this work. The skills I have learned from him are highly invaluable and will surely stay with me for life. Dr. Loveless has done so much to ensure that I have a very successful future ahead of me and I can't thank him enough for that. I would like to thank Dr. Loveless, Dr. Reising, and Dr. Sreenivas for their reviewing this work and providing input to ensure that this work is presented as strongly as it can be.

I would like to thank my family for their undying support during my many stressful moments in graduate school. I truly would not have made it through without you all. Thanks as well to my sister Rachel and brother-in-law Tyler for housing me during much of my graduate school experience. 


\section{TABLE OF CONTENTS}

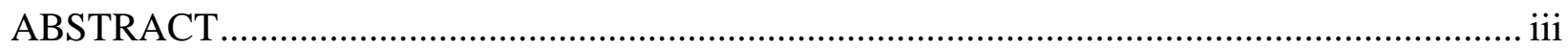

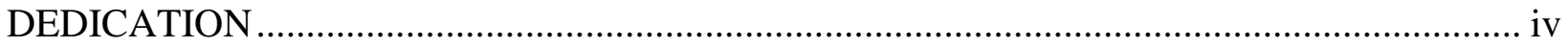

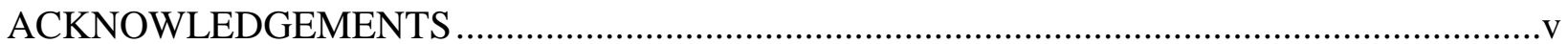

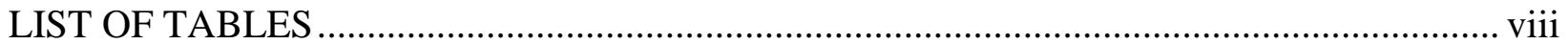

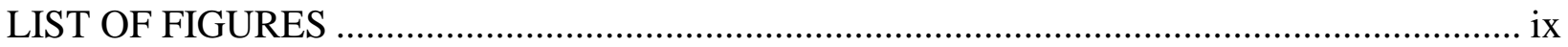

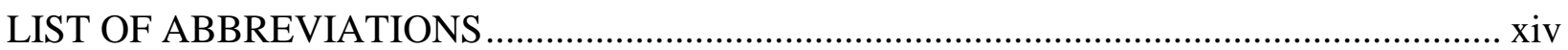

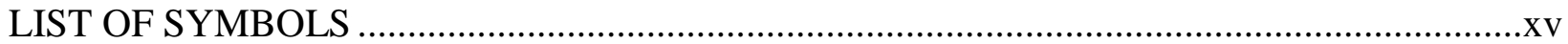

\section{CHAPTER}

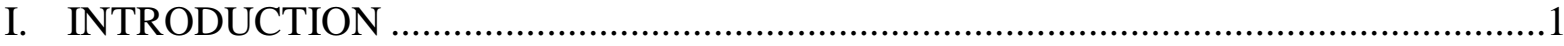

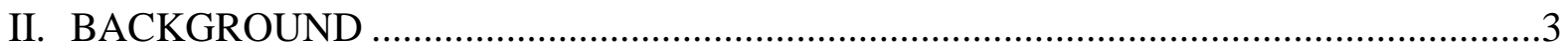

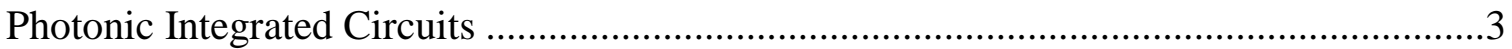

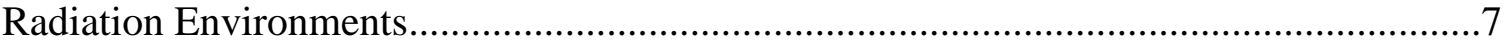

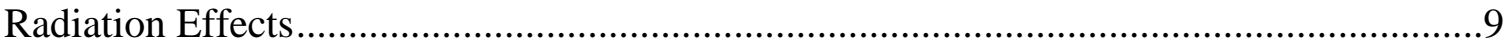

Single-Photon Absorption and Two-Photon Absorption ................................................10

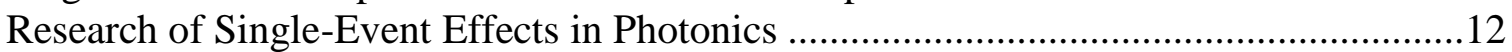

The Physics of Charge Transport Simulations................................................................17

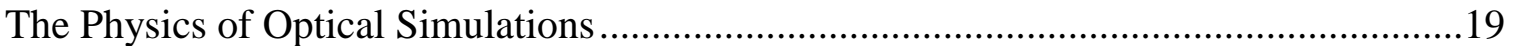

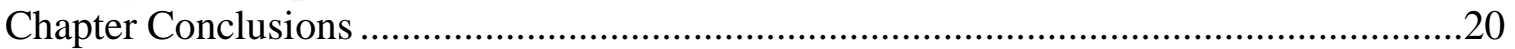

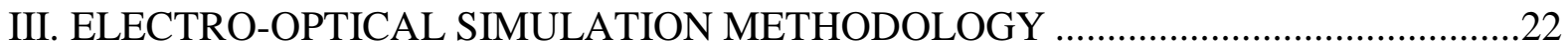

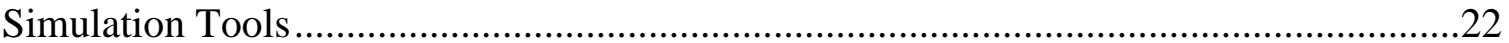

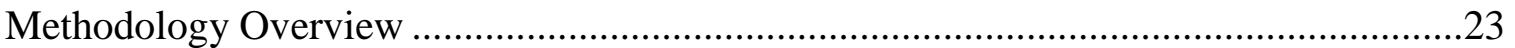

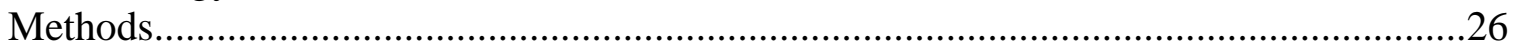

Stage A - Emulation of Heavy Ion Strike in Optical Domain.........................................29

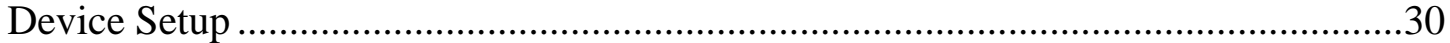

Simulation Region Setup ……………………………....................................31

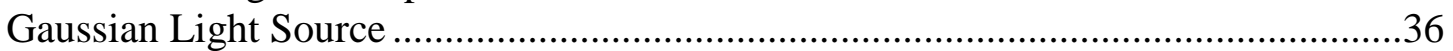




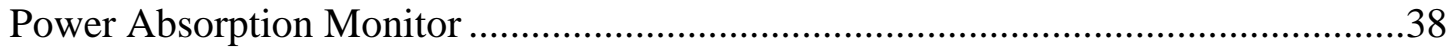

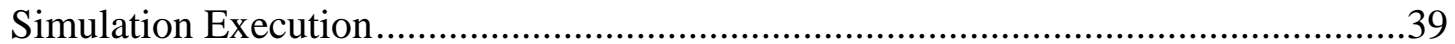

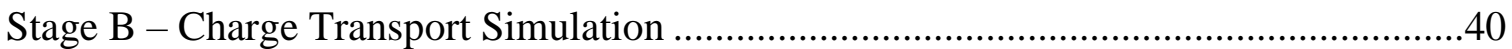

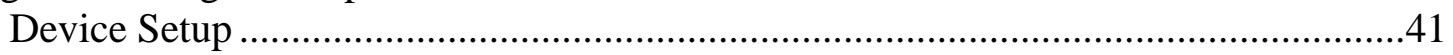

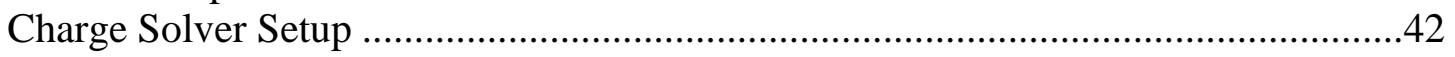

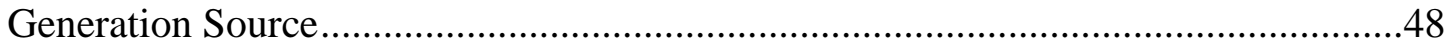

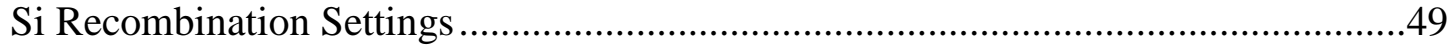

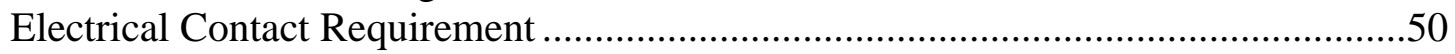

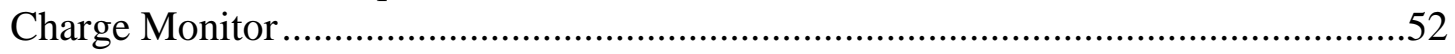

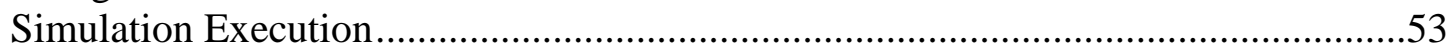

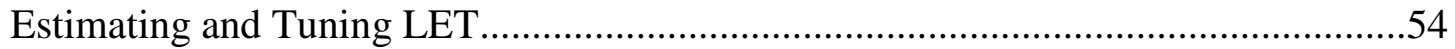

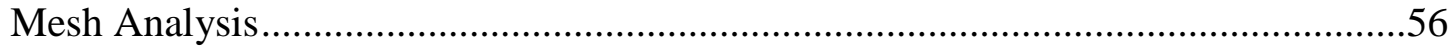

Stage C - Extraction of Losses, Phase Shift, and Optical S-Parameters .........................57

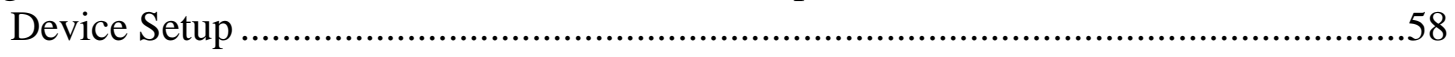

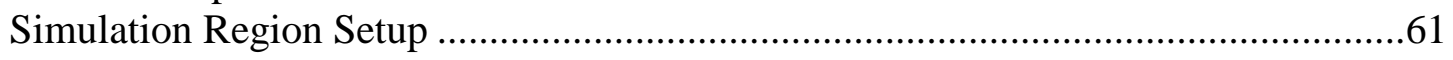

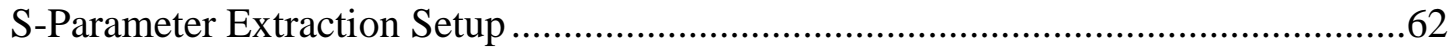

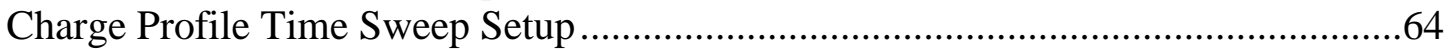

Simulation Execution and Result Processing .....................................................66

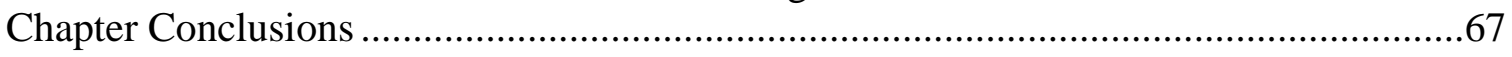

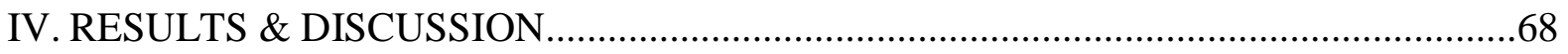

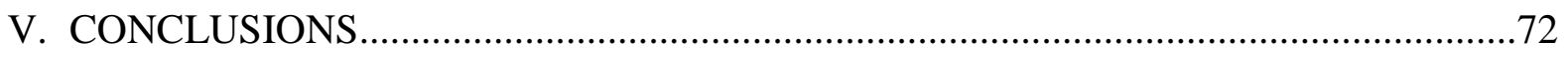

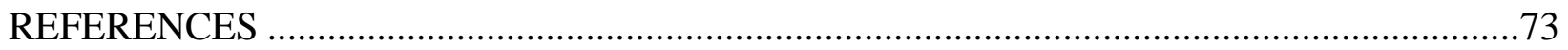

\section{APPENDIX}

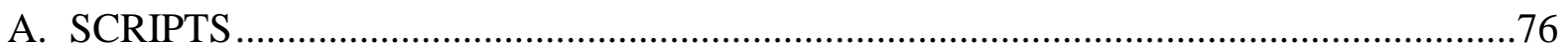

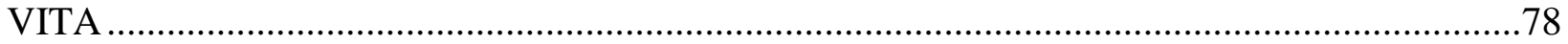




\section{LIST OF TABLES}

1 Coefficients of the Soref and Bennett charge-to-index conversion model for an optical

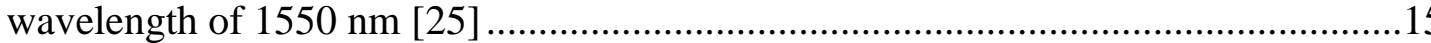

viii 


\section{LIST OF FIGURES}

1 Demonstration of light transmission through a waveguide. The interface between the $\mathrm{Si}$ and $\mathrm{SiO} 2$ creates a reflective boundary that allows the injected light to be guided along the path of the waveguide

2 Transmission modes of optical waveguides. Modes may take on various shapes that depend on the waveguide dimensions, the optical properties of the waveguide material, the carrier wavelength. Left illustrates a transverse magnetic (TM) mode and right illustrates a transverse electric (TE) mode. A TM mode has its magnetic field transverse (or perpendicular) to the direction of propagation and its electric field aligned in the direction of propagation. A TE mode is the opposite having its electric field transverse and its magnetic field in the direction of propagation

3 The near-earth radiation environment consists of radiation from various sources, including the belts of trapped particles surrounding the earth, solar activity and events, and galactic cosmic rays (GCRs) that occur outside of the solar system [2] ....8

4 An ion striking a Si PN junction causes the generation of free carriers that are swept away by the electric field in the depletion region (left) and causes a current transient at the circuit nodes connected to the PN junction (right) [3]

5 The optical absorption coefficients for single-photon absorption (obtained from [4]) and two-photon absorption (obtained from [5]) in Si

6 A graph of Si refractive index versus EHP density for the Soref and Bennet charge-toindex conversion model. As EHP density increases in $\mathrm{Si}$, decreases in refractive index are observed. As the EHP density rises to about $1.83 \mathrm{e} 21 \mathrm{~cm}^{-3}$ the refractive index goes below 1 and starts to become reflective and absorbing like a metal [6]....14

7 Trend of waveguide transmission losses versus LET with trendline from [6]. These values were computed in Lumerical FDTD for static charge profiles in a slab waveguide of cross-sectional dimensions of $220 \mathrm{~nm}$ wide by $450 \mathrm{~nm}$ high

8 The mechanism of single-event radiation effects in a Si waveguide. Charges induced at the ion strike location cause shifts in the Si's refractive index. Significant free charges cause the Si to become reflective and absorbing

9 Flowchart of the proposed simulation methodology that makes it possible to simulate the full time-domain effect of an ion strike in any arbitrary optical device. In A) an optical domain simulation is performed to emulate the effect of an ion striking the 
device. A power absorption profile is computed, and this is passed to stage B) where an impulse of charges is generated, diffused, and recombined to capture the electronic effects of the ion strike. A set of discrete-time 3D charge profiles are then generated, and these are passed to stage C) where each profile is used to compute the shifts in optical phase and power loss that represent the overall device response to the ion strike. While this figure shows Lumerical FDTD and DEVICE being used for the optical and electrical domains respectively, this methodology should generally work with any optical and electrical simulation tools with similar features

10 A cross-sectional slice of the simulated Si slab waveguide. The waveguide is $700 \mathrm{~nm}$ wide by $220 \mathrm{~nm}$ high. This $\mathrm{Si}$ slab is surrounded entirely by $\mathrm{SiO} 2$ to create the reflective barrier required for guiding the light waves. The $\mathrm{SiO} 2$ is split into separate a separate surface oxide and buried oxide

11 The normalized electric field intensity of the fundamental TE mode of the simulated waveguide shown in Figure 10

12 The 3D model of the Si waveguide in Lumerical FDTD. The white arrow represents the location and direction of the simulated ion strike using SPA

13 The generated mesh surrounding the ion strike location in Lumerical DEVICE. The mesh gets finer in the regions closer to the center of the strike location to prevent fast changing spatial gradients in the charge density from causes the simulation to diverge.

14 The object tree in Lumerical FDTD with the tools required for the ion strike simulation. The objects named "waveguide", "buried oxide", and "surface oxide" are the shapes used to define the dimensions and materials of the waveguide in this example. "FDTD" is the FDTD solver object that defines the simulation region and mesh and the object called "mesh" is the mesh override object that is defining a finer mesh directly surrounding the ion strike location. Unique to this simulation is the Gaussian light source (called "Strike") and the power absorption monitor (called "Pabs")

15 Defined dimensions of the FDTD simulation region for the ion strike simulation. This region encapsulates the waveguide of Figure 10. The simulation region has been defined as $1.5 \times 5 \times 0.44 \mu^{3}$.

16 Defined dimensions of the FDTD simulation region for the ion strike simulation. This region encapsulates the waveguide of Figure 10. The simulation region has been defined as $1.5 \times 5 \times 0.44 \mu \mathrm{m}^{3}$.

17 The boundary condition settings in Lumerical FDTD. The boundary condition at each boundary ("x min bc", "x max bx", "y min bc", etc.) is set to perfectly matched layer (PML). The PML settings are set to their defaults. 
18 Mesh override settings for the region directly surrounding the ion strike. This defines the maximum mesh step size to be $5 \mathrm{~nm}$ along all axes. This guarantees that the generated power absorption profile will have a sufficiently fine resolution

19 The location and dimensions of the mesh override region

20 The gaussian source geometry settings. It is set to be a "z-normal" source so that it injects light in the $\mathrm{z}$ direction and is set to be a diameter of about $50 \mathrm{~nm}$ with " $\mathrm{x}$ span" and "y span". The z coordinate is set to $220 \mathrm{~nm}$ to place it on top of the waveguide

21 The gaussian light source wavelength settings. "Wavelength start" and "wavelength stop" have been set as to the same wavelength of $500 \mathrm{~nm}$ to achieve as close as possible to a pure $500 \mathrm{~nm}$ light signal

22 The power absorption monitor can be found in Lumerical FDTD's Object Library under "Optical Power". The non-advanced option is all that is necessary for these simulations

23 A cross-section of the waveguide showing the optical power absorbed frome the light source at the point where it is at full intensity. This power absorption profile gets converted to an electron-hole pair generation rate profile for use in the Lumerical DEVICE simulation to act as an emulation of an ion strike in the electrical domain simulation.

24 The Objects Tree in Lumerical DEVICE for the charge transport simulation

25 The general charge solver settings. This set to a 3D transient simulation at standard room temperature

26 The charge solver mesh settings. The minimum mesh edge length is $0.1 \mu \mathrm{m}$ and the maximum mesh edge length is set to $10 \mu \mathrm{m}$. A long max edge length allows the simulator to generate a mesh that is very coarse in regions far away from the ion strike location to save computational resources. "Max refine steps" has been set to 200000 so that the mesh generator can thoroughly refine the mesh

27 The dimensions and settings for the mesh constraint region directly surrounding the ion strike location. This region prevents simulation divergence by making the mesh finer where the charge gradients are expected to be the steepest

28 The transient simulation settings for the charge solver. (a) has the minimum time step setting for the simulation set to $1000 \mathrm{fs}$ and the maximum time step set to $10 \mu \mathrm{s}$. (b) is the set of settings that determines the timing of the pulse of charges generated by the charge generation source. (c) scales the intensity of the charge pulse generated such that LET may be tuned in this methodology .46

29 Adding an optical generation source to the simulation region .48 
$30 \mathrm{Si}$ recombination settings of the charge solver. The various type of recombination in $\mathrm{Si}$ may be enabled and disabled here. The default settings of each type of recombination are already set appropriately for $\mathrm{Si}$

31 Dimensions and placement of the aluminum contact. It's width in the $\mathrm{x}$ direction is 600 $\mathrm{nm}$ and it is placed directly under the strike location

32 Lumerical DEVICE electrical contact settings. To define a shape in the simulation region as an electrical contact, (a) was set to a solid geometry and (b) was then set to the specific solid name that the user wishes to define as the contact (here it is set to the "contact" as seen in Figure 24). (c1) was set to initial time and voltage of the contact, while (c2) was set to the simulation end time and voltage. Both voltages were set to 0 to ensure that the electrical contact does not affect the passive diffusion simulation in this example.

33 The settings of the charge monitor. "Record electrons", "record holes", "save data", and "integrate total charge" were all checked. A filename was also specified so that the charge profiles recorded by the monitor would be saved

34 An example set of charge profiles computed by Lumerical DEVICE. As time progresses, the charge spreads out through the waveguide and the peak charge density decreases. These discrete-time charge profile steps are used in the following simulation to compute the transmissive parameters of the waveguide for each time step. Note that the color scale is renormalizing for each time step in this image. This helps to better visualize the diffusive nature of the charge.

35 The settings of the charge monitor. Both "record electrons" and "record holes" must be selected. "Save data" allows the charge monitor to automatically save the computed charge profile to a file named in the "filename" field. "Integrate total charge" computes the total number of electrons and holes within the charge monitor which is convenient for estimating LET

36 Estimated LET versus maximum mesh length for an example charge transport simulation.

The estimated LET converges to an LET of about $97.7 \mathrm{MeV}-\mathrm{cm}^{2} / \mathrm{mg}$ as maximum mesh length is reduced, indicating that the simulation becomes more accurate with reduced mesh size. LET seems to be over-estimated at max mesh lengths larger than $5 \mathrm{~nm}$

37 The Objects Tree in Lumerical FDTD for the s-parameter extraction simulations. The waveguide and mesh is setup identically to the device in Figure 14. There is now an "np density" object for including the carrier profiles in FDTD. The FDTD simulation object now includes input and output ports that facilitate the extraction of device's s-parameters

38 The properties of the "np density" object. This object allows the user to import a set of charge profiles generated by Lumerical DEVICE through the "Import data..." button 
39 The Si material settings for modeling the shifts in optical properties. A new Si material has been created "Si - IP" and has been set to a material type of "index perturbation" and set to inherit the optical properties of the "Si $(\mathrm{Si})$ - Palik" material. To enable Si's refractive index dependency on free carriers, "include np density" is checked and the chosen model is the "Soref and Bennett model" with $1550 \mathrm{~nm}$ coefficients

40 The FDTD simulation region dimensions for the parameter extraction simulation. The length of this simulation region (y-direction) is set to $51 \mu \mathrm{m}$ so that it is slightly longer than the imported charge profile. The other dimensions are identical to the simulation region in Figure 15 making it a $1.5 \mu \mathrm{m}$ x $51 \mu \mathrm{m} \times 0.44 \mu \mathrm{m}$ simulation region

41 S-Parameter extraction input and output port dimensions. These ports are placed at opposing ends of the waveguide and are made slightly smaller than the overall simulation region but still larger than the cross section of the waveguide so that the ports can generate the whole waveguide mode.

42 The modal properties of the input port. This port is set to inject forward along the y-axis so that it injects in the positive y direction. The amplitude and phase are set to 1 and 0 respectively to simply act as a normalized electromagnetic wave. The source is operating in the waveguide's fundamental mode over 1 frequency point $(1550 \mathrm{~nm})$

43 This parameter sweep adjusts the value of the time index of the np density charge profile to perform a series of simulations that captures the device's s-parameters as they shift over time due to the changing charge profile. Here the sweep has been setup such that the user may specify the values of time index to be simulated. This parameter sweep outputs the s-parameters as a function of the time index

44 The transient transmission characteristics of the waveguide in Figure 10 for three LET values computed via the simulation methodology presented in this work. As LET increases, the intensity of the transients becomes more severe, reaching as high as $1 \%$ transmission loss and -0.015 radians for an LET of $40 \mathrm{MeV}-\mathrm{cm}^{2} / \mathrm{mg}$

45 The peak values of transmission loss and phase shift versus LET. This graph appears to show a somewhat linear relationship between LET, peak transmission loss, and peak phase shift in the waveguide

46 An example of a silicon photonic modulator. A similar structure may be useful for draining single event induced charges out of a waveguide and therefore reducing the time constant of the effect induced by these charges. [7] 


\section{LIST OF ABBREVIATIONS}

CMOS, Complementary Metal Oxide Semiconductor

EHP, Electron-Hole Pair

IC, Integrated Circuit

Si, Silicon

$\mathrm{SiO} 2$, Silicon Dioxide

PIC, Photonic Integrated Circuit

TE, Transverse Electric

TM, Transverse Magnetic

TID, Total Ionizing Dose

SEE, Single-Event Effect

LET, Linear Energy Transfer

SET, Single-Event Transient

SEU, Single Event Upset

FDTD, Finite-Difference Time-Domain

SPA, Single-Photon Absorption

TPA, Two-Photon Absorption

PML, Perfectly Matched Layer 


\section{LIST OF SYMBOLS}

$N$, EHP Concentration

$\alpha$, SPA Coefficient

$\beta$, TPA Coefficient

$\hbar$, Reduced Planck's Constant

$\omega$, Angular Frequency

$I$, Irradiance

$\Delta n$, Change in Refractive Index

$\Delta \alpha$, Change in Absorption Coefficient

$\boldsymbol{E}$, Electric Field

$\phi$, Electrostatic Potential

$n$ and $p$, Electron and Hole Concentration Respectively

$N_{d}$, Donor Density

$N_{a}$, Acceptor Density

$q$, Fundamental Charge/Charge of One Electron

$\epsilon_{0}$, Vacuum Permittivity

$\boldsymbol{J}_{\boldsymbol{n}}$ and $\boldsymbol{J}_{\boldsymbol{p}}$ Electron and Hole Current Density Respectively

$\mu_{n}$ and $\mu_{p}$, Electron and Hole Mobility Respectively

$D_{n}$ and $D_{p}$, Electron and Hole Diffusion Coefficient Respectively

$k_{B}$, Boltzmann's Constant 
$T$, Temperature

$G_{n}$ and $G_{p}$, Electron and Hole Generation Rate Respectively

$R_{n}$ and $R_{p}$, Electron and Hole Recombination Rate Respectively

$\rho$, Local Charge Density

$\boldsymbol{H}$, Magnetic Field

$\epsilon_{r}$, Relative Permittivity

$T_{\text {loss }}$, Transmission Loss

$\phi_{\text {shift }}$, Phase Shift

$s(t)$, Time-Dependent Complex S-Parameters 


\section{CHAPTER I}

\section{INTRODUCTION}

Ionizing radiation poses a challenge to designers of integrated circuits for space environments. The bombardment of highly energetic particles such as protons and heavy ions with semiconductor material can cause erratic behavior in electronic device, circuit, and system performance. The study of this erratic behavior in radiation-rich environments, known as radiation effects, is vital to understanding the mechanisms that cause such changes to occur, the modeling of these effects in circuits and devices, and the development of techniques that help mitigate these effects.

Radiation effects in Complementary Metal Oxide Semiconductor (CMOS) transistor technology have been studied for decades, and this long-standing research has informed and guided the design of reliable CMOS integrated circuits (IC)s for use in space systems as well as other radiation harsh environments. More recently, research has shown that photonic integrated circuits (PICs) may also be affected by ionizing radiation $[1,2,3,4,5]$. This discovery has opened up a whole new field of radiation effects research in photonic devices that is still in its infant stages. Investigating the effects of radiation on PICs is further motivated by their growing list of potential applications in space systems, including communications, sensing, autonomous navigation, and imaging [6].

Radiation effects are broken down into two primary subclasses of study: Total ionizing dose (TID) and single-event effects (SEEs). TID is caused by continued exposure of circuits to 
ionizing radiation and may cause the physical parameters of devices to degrade, such as transistor threshold voltages and transconductance, due to trapped charge in the transistor oxides [7]. SEEs on the other hand are the result of the interactions of single ionizing particles with semiconductor junctions, which may cause current and voltage fluctuations (called a single-event transient or SET) capable of corrupting data stored in the circuit. The severity of a SET depends on the charge generated and collected within a semiconductor junction and the response of the circuit to that transient current [8].

Until now, radiation effects studies on PICs have been primarily limited to studies of TID $[1,3,4,5]$, however, recent studies have demonstrated that SEEs may also be a significant area of study for PICs [2]. In particular, there is currently a lack of ability to simulate and model the time domain transient effects that occur as a result of an ionizing particle striking an optical component. The difficulty of simulating SEEs in PICs arises from the requirement to couple electrical domain and optical domain simulations such that the full interaction between photon absorption and reflection, electron-hole pair (EHP) generation, and charge transport can be captured in the results, and give an accurate portrayal of the transient disturbance caused by the single-event.

This thesis presents a simulation methodology that closes the gap between the electrical and optical domains to simulate SETs in individual optical devices and provide details of the transient phase shift and power loss seen at the output of the device and how these device characteristics shift as the SEE propagates and then dissipates. These results allow for the development of models of SEEs in PICs at various ion energy levels as well as for various device fabrication dimensions and materials. Consequently, methods for improving the radiation hardness of photonic devices may be discovered through the analysis of these models. 


\section{CHAPTER II}

\section{BACKGROUND}

This chapter starts by introducing the reader to the fundamental concepts of PICs including basic transmission and modal theory. Then there is a brief section on space radiation environments followed by an introduction to the mechanisms of SEEs in conventional electronics. The current state of research on radiation effects in PICs is then described to illustrate the reasons for developing a simulation methodology for SEEs in PICs. Finally, the theory behind electronic charge transport and optical FDTD simulations is described.

\section{Photonic Integrated Circuits}

Conventional electronics have facilitated the transmission and processing of electrical signals for more than a century. More recently, however, photonic technology has allowed for the transmission and processing of optical (light) signals that yield much higher data bandwidths, reaching into the tens of Gbps per channel with the possibility of scaling into the range of Tbps if parallel channels are implemented [9]. In addition to the commercial applications, photonics is also expected to see increased use in space systems to augment the performance of conventional electronic systems [6]. Conveniently, the same silicon ( $\mathrm{Si}$ ) technology that allows the fabrication of CMOS transistors, also facilitates the fabrication of Si photonic integrated circuits. This may allow for full photonic/electronic systems to be implemented on the same chip, assuming that the challenge of fabricated lasers on a chip can be overcome. 
The primary component of any photonic system is the waveguide. Waveguides act as the means of transporting information in an optical system. Any optical waveguide requires at least two materials to function: A core material transparent to the carrier wavelength, and a bounding material that will reflect the carrier to continuously guide it within the waveguide core. With current $\mathrm{Si}$ photonic circuit technology, it is possible to fabricate a basic waveguide as a slab of $\mathrm{Si}$ (the transparent core material) surrounded by silicon dioxide ( $\mathrm{SiO} 2)$ (the bounding material). $\mathrm{Si}$ has the property of being transparent at optical wavelengths as low as $1100 \mathrm{~nm}$. This makes the best transmission range of Si photonics within the infrared spectrum. In fact, the standard carrier wavelength for Si photonic systems is $1550 \mathrm{~nm}(193.4 \mathrm{THz})$.

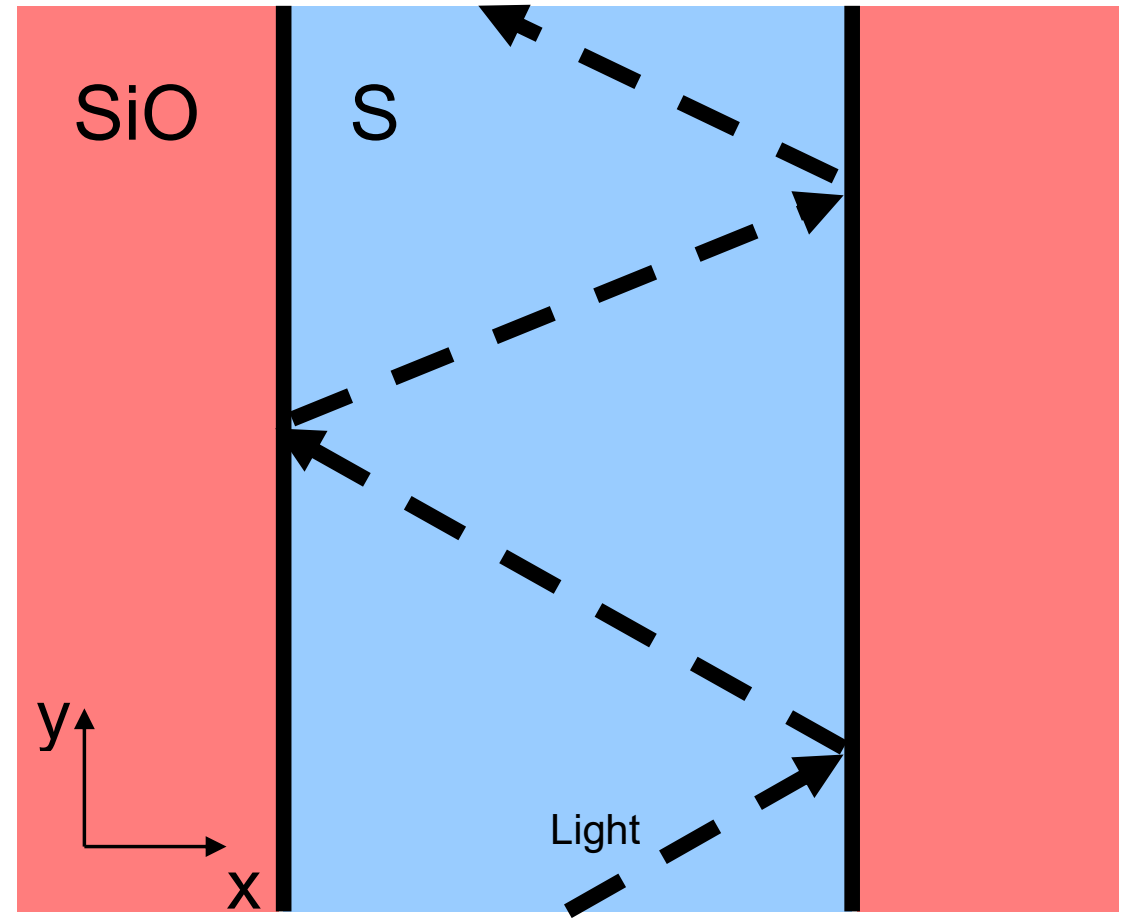

Figure 1

Demonstration of light transmission through a waveguide. The interface between the Si and $\mathrm{SiO} 2$ creates a reflective boundary that allows the injected light to be guided along the path of the waveguide 
Although $\mathrm{SiO} 2$ is also transparent to these wavelengths, the theory of total internal reflection allows the light to still be reflected back into the Si at the boundary between the Si and $\mathrm{SiO} 2$ [10] (Figure 1). This occurs due to the difference in refractive index between the materials where the Si has refractive index 3.48 at $1550 \mathrm{~nm}$ [11] and the $\mathrm{SiO} 2$ has refractive index of 1.44 at $1550 \mathrm{~nm}$ [12]. Note that the refractive index of the bounding material ( $\mathrm{SiO} 2)$ is lower than that of the core material (Si). This is necessary for total internal reflection to guide the light through the core material.

As the wave reflects between the boundaries of the waveguide, standing wave patterns are created along the directions perpendicular to the direction of propagation. These standing wave patterns (illustrated in Figure 2), referred to as modes of propagation, are derived as solutions to the wave equation resulting from Maxwell's equations. The shape and type of mode is dependent upon the physical dimensions of the waveguide and the wavelength of the carrier signal that is being propagated down the waveguide. The mode profile may be used as a means of determining how well guided the mode is, that is how much of the signal energy falls within the waveguide itself, and how much gets radiated and lost in the $\mathrm{SiO} 2$. The more the mode profile falls outside of the waveguide, the more optical energy is lost per unit length of waveguide.

One way to categorize modes is by their order. A $1^{\text {st }}$ order mode is excited in the waveguide if the mode profile has a single peak at the center of the waveguide (Figure 2). This $1^{\text {st }}$ order mode is also called the fundamental mode. A $2^{\text {nd }}$ order mode may be excited, which consists of two peaks of opposite polarity. A $3^{\text {rd }}$ order mode may be excited that has three peaks in the field. This trend of modes may in theory continue indefinitely; however, higher order modes tend to be less well guided and thus subject to higher power losses. Higher order modes may often be excited as artifacts of the excitation of the fundamental mode in an imperfect waveguide [13] or they may be 
intentionally excited via a device called a mode transducer that converts one mode to another [14]. These modal orders are analogous to the vibrational modes of vibrating strings that are tied at each end. A waveguide should be designed such that each of its physical dimensions are equal to a multiple of half the carrier wavelength as this allows for the modes to have minimal losses.
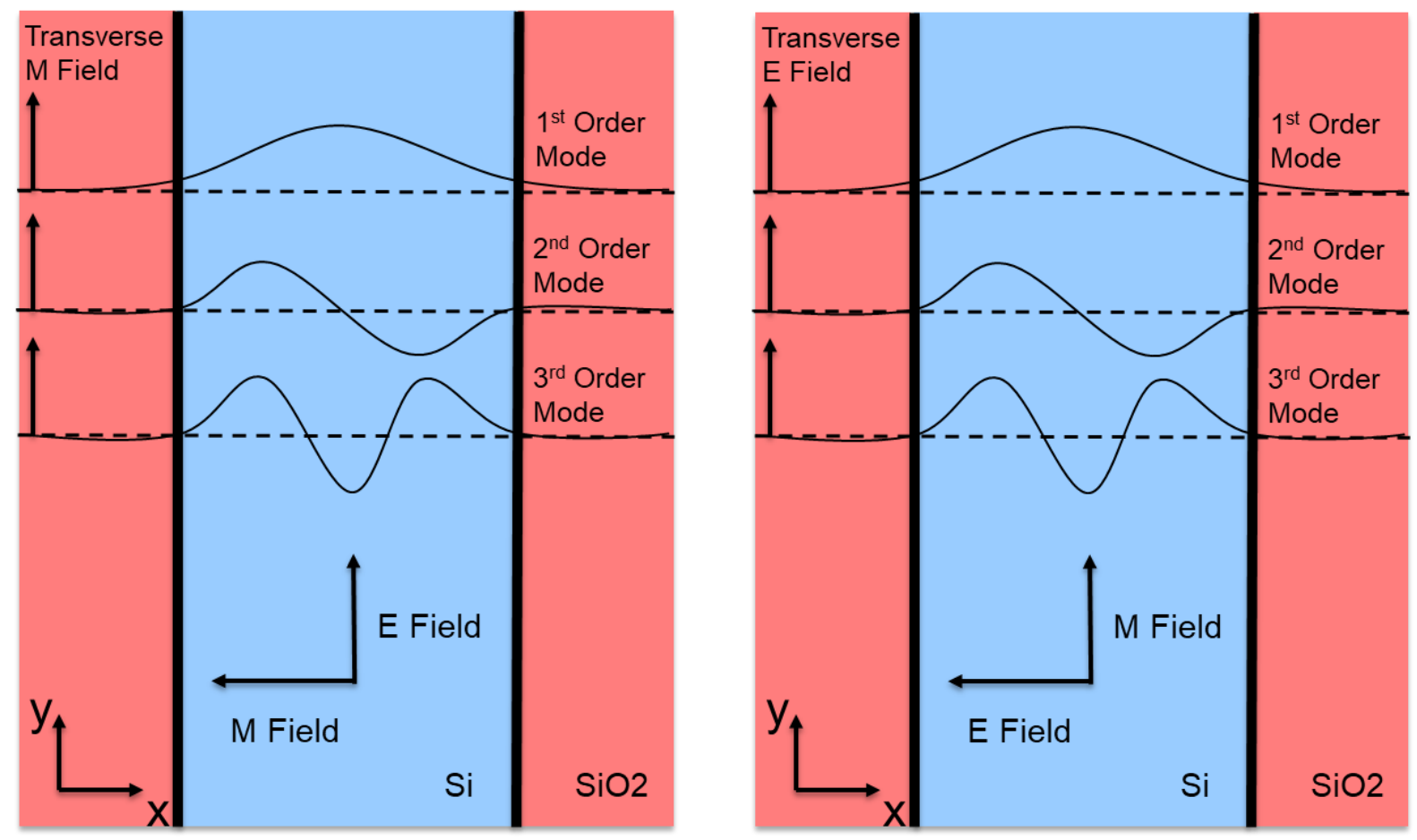

Figure 2

Transmission modes of optical waveguides. Modes may take on various shapes that depend on the waveguide dimensions, the optical properties of the waveguide material, the carrier wavelength. Left illustrates a transverse magnetic (TM) mode and right illustrates a transverse electric (TE) mode. A TM mode has its magnetic field transverse (or perpendicular) to the direction of propagation and its electric field aligned in the direction of propagation. A TE mode is the opposite having its electric field transverse and its magnetic field in the direction of propagation

Modes are also categorized by their transverse field [15]. A transverse electric (TE) mode will have its electric field polarized such that it is transverse (perpendicular) to the direction of 
propagation, and its magnetic field will be polarized in the direction of propagation. A transverse magnetic (TM) mode is the opposite of TE, with its magnetic field polarized transverse to the direction of propagation and its electric field polarized in the direction of propagation. A pure TE or TM mode is only possible in theory. In a physical waveguide, a mode manifests itself as some superposition of both TE and TM, though it is possible to get close to pure TE or TM.

PICs include a variety of components other than waveguides. Passive components include waveguides, ring resonators, interferometers, and couplers, for example. There are also active components such as modulators, optical amplifiers, lasers, and photodiodes. Though there are numerous types of optical components, most of them are fabricated as different structures of waveguides, such as ring resonators, modulators, directional couplers, and many more. Therefore, to make this research generalizable as possible to all optical components, this thesis focuses solely on the response of waveguides to ionizing radiation.

\section{Radiation Environments}

The design of electronic and photonic devices that operate within the earth's troposphere generally requires little concern for radiation effects. Some of the limited exceptions to this would be systems that operate within or near radiation test facilities and high energy particle accelerators [1]. However, as systems leave the earth's atmosphere, they enter a space environment filled will radiation from a variety of sources (Figure 3) [11].

One reason for the lower earth atmosphere having limited exposure to radiation is that the earth has a magnetic field that traps ionizing radiation that comes from solar flares, effectively shielding the earth from being struck with ionizing particles. The regions in which the radiation becomes trapped are known as the Van Allen Belts. These radiation belts pose a problem to 
electronic systems that pass through them to travel beyond the earth as well as for satellites that orbit the earth and must travel through the belts continually.

Further away from the earth is an environment susceptible to radiation that comes directly from the sun. During a solar flare, it is likely that a system will be struck by many protons and heavy ions. Another radiation concern in this environment comes in the form of galactic cosmic rays (GCR) which are high energy particles that are traveling at significant fractions of the speed of light. GCRs originate from outside of the solar system from events elsewhere in the galaxy such as supernovas. [16]

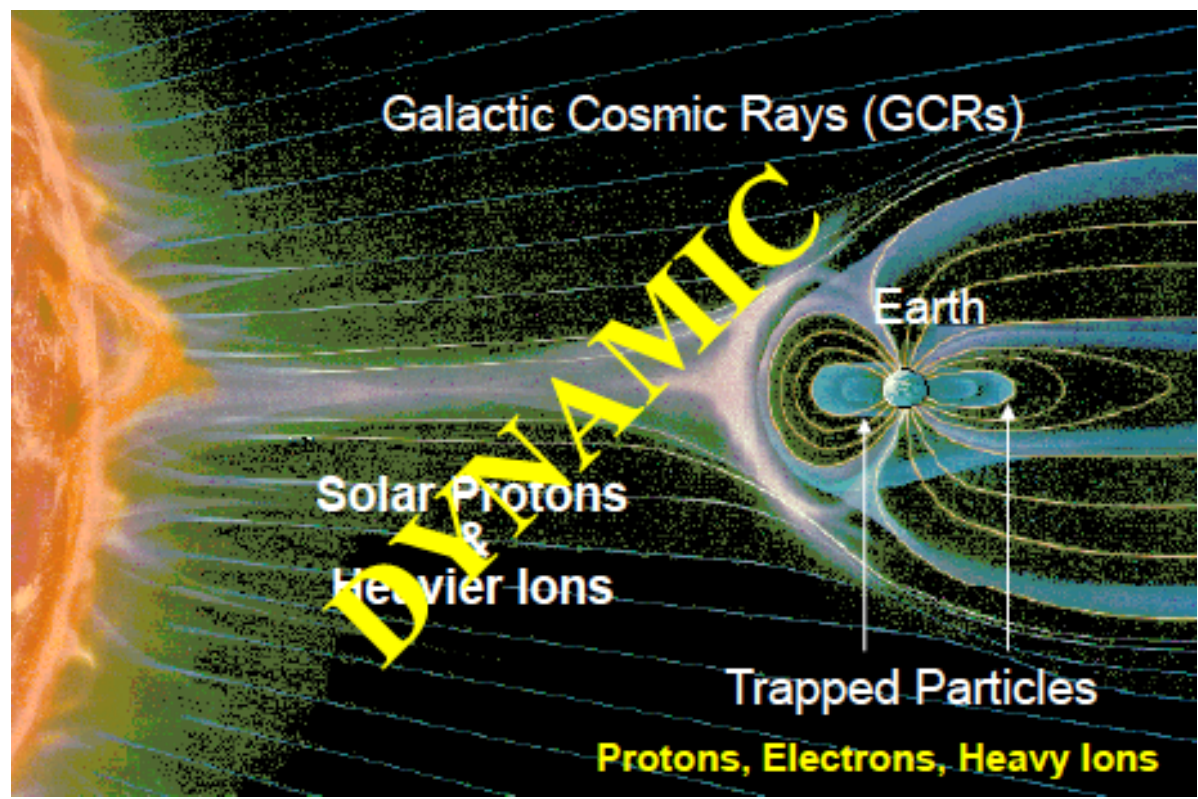

Figure 3

The near-earth radiation environment consists of radiation from various sources, including the belts of trapped particles surrounding the earth, solar activity and events, and galactic cosmic rays (GCRs) that occur outside of the solar system [17] 


\section{Radiation Effects}

When ionizing particles strike semiconductor material, they transfer energy to the system in the form of generating free charges in the material. There are two primary subclasses of effects that these free charges may have on a system. One type occurs when a circuit is under long-term exposure to ionizing radiation and extraneous positive charges get trapped in the device oxides. This effect is due to the low mobility of holes in the oxides. In CMOS technology, these trapped excess charges have the effect of degrading device parameters such as transistor threshold voltages and gate capacitances. The severity of the effect is proportional to the amount of radiation exposure in both time and intensity and is classified by the total dose which is measured in units of $\mathrm{kRads}(1 \mathrm{kRad}=10 \mathrm{~J} / \mathrm{kg})$. As such, these types of effects are called total ionizing dose (TID) effects.

The second subclass of radiation effect is the instantaneous transient effect that occurs when a single ionizing particle strikes a component (known as single-event effects or SEEs). In CMOS technology, the free charge that is generated by the ion strike can be collected at nearby PN junctions through the electric field in the depletion region (Figure 4) [12]. This results in a current pulse at each of the affected circuit nodes. The severity of this effect depends on the charge that is generated by the ion strike, the total charge collected at the circuit node, and the response of the circuit itself.

The amount of charge that is generated in the device depends on the total energy that is transferred from the ion to the semiconductor material. In the study of SEEs, this transfer is quantified by the linear energy transfer (LET) of a particle (measured in units of $\mathrm{MeV} \mathrm{cm} / 2 / \mathrm{mg}$ ). Due to the generation of larger amounts of free carriers, higher LET values equate to a larger effect on the overall circuit. 
The current pulse that is generated in the device junctions in response to the ion strike causes a perturbation in the voltage at the affected nodes that is known as a single-event transient (SET). The intensity of a SET is highly dependent on the capacitance at the affected node, lower node capacitances being more susceptible to SETs due to them requiring less total charge to perturb the voltage. If a SET is severe enough to cause a bit to flip in a circuit that results in information corruption, this is known as a single-event upset (SEU).
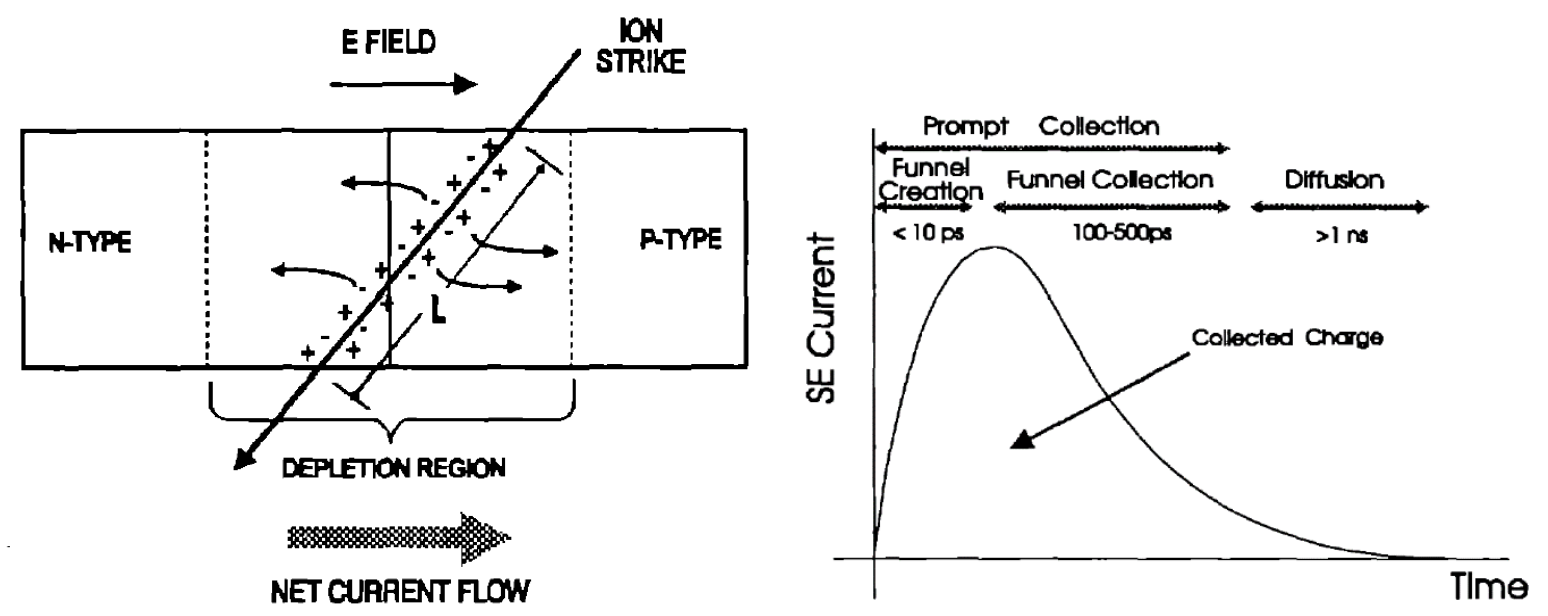

Figure 4

An ion striking a Si PN junction causes the generation of free carriers that are swept away by the electric field in the depletion region (left) and causes a current transient at the circuit nodes connected to the PN junction (right) [18]

\section{Single-Photon Absorption and Two-Photon Absorption}

Laser pulses are often injected into electronic devices to emulate the effect of an ion striking the device $[19,20]$. This is because photons absorb into silicon to generate EHPs similarly to how the transfer of energy from ionizing particles may generate EHPs during a single event. There are two distinct processes by which lasers are used to induce charges in silicon: single- 
photon absorption (SPA) and two-photon absorption (TPA). In SPA, a single photon interacts with the material to generate a single EHP. In TPA, two photons interact with the material simultaneously to generate a single EHP.

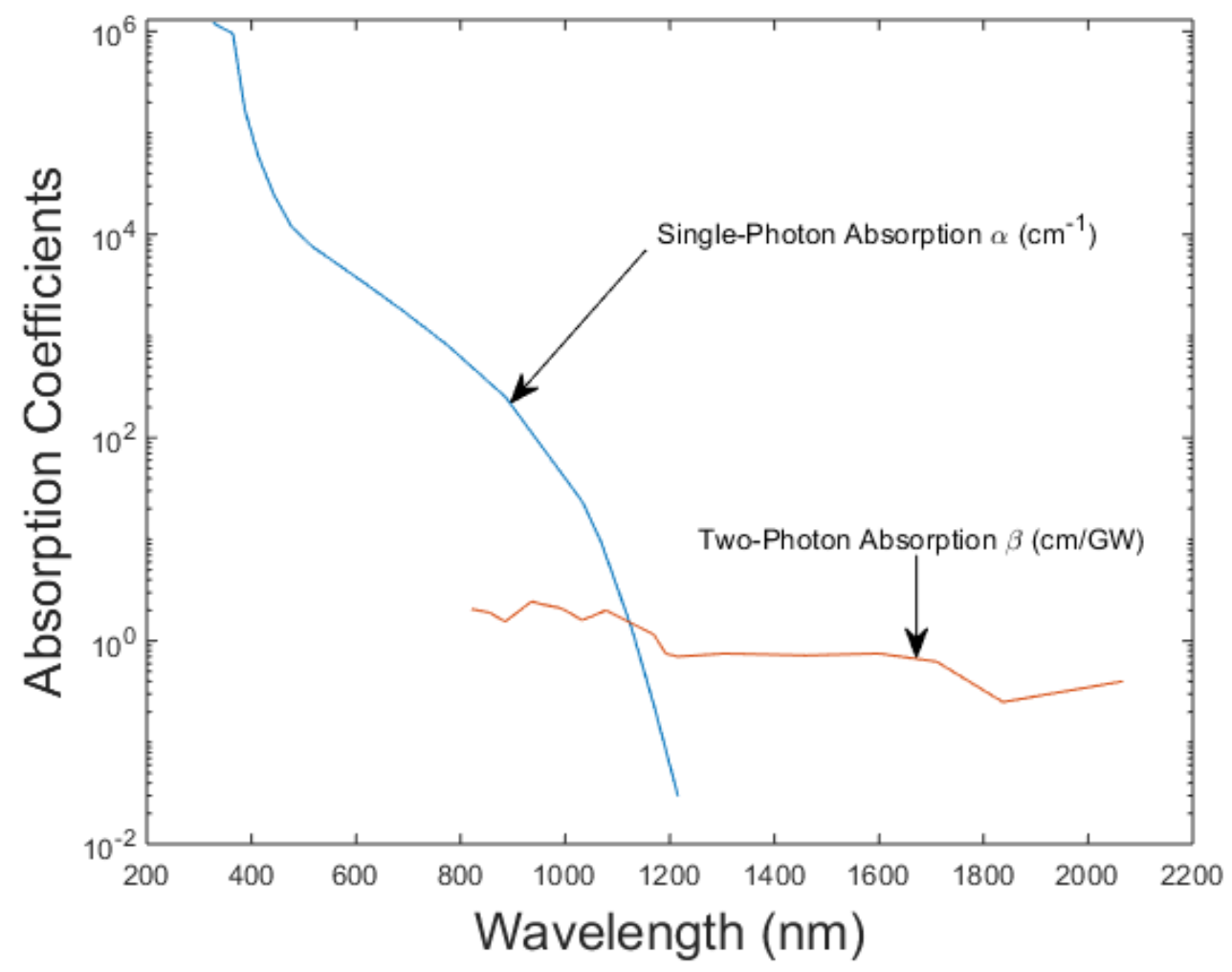

Figure 5

The optical absorption coefficients for single-photon absorption (obtained from [21]) and twophoton absorption (obtained from [22]) in $\mathrm{Si}$

The equation describing the combined effect of SPA and TPA is shown below [19].

$$
\frac{d N}{d t}=\frac{\alpha(\lambda) I}{\hbar \omega}+\frac{\beta(\lambda) I^{2}}{2 \hbar \omega}
$$

In Equation 2.1, $\mathrm{N}$ is the concentration of generated EHPs in $\mathrm{cm}^{-3}, \alpha(\lambda)$ is the wavelength dependent SPA coefficient in $\mathrm{cm}^{-1}, \beta(\lambda)$ is the wavelength dependent TPA coefficient in $\mathrm{cm} / \mathrm{GW}$, 
and $I$ is the irradiance. The values of $\alpha(\lambda)$ (obtained from Jones et. al. [21]) and $\beta(\lambda)$ (obtained from Bristow et. al. [22]) are plotted versus wavelength in Figure 5. The coefficients of SPA and TPA intersect at a wavelength of about $1150 \mathrm{~nm}$ which is about the wavelength of a photon whose energy equals the Si bandgap of $1.1 \mathrm{eV}$. To the left of this point, for small wavelengths, the SPA mechanism dominates since the energy of each photon is greater than the band gap of Si. For wavelengths greater than $1150 \mathrm{~nm}$, the TPA mechanism dominates because two photons are required to efficiently generate EHPs.

According to Buchner et. al. [19], both SPA and TPA are useful for emulating ion strikes in silicon devices, however, SPA doesn't allow for the injection of carriers at variable depth, which can be a problem when there are other fabrication layers blocking the laser from reaching the device. This issue may be solved by using a laser with a TPA wavelength (above $1150 \mathrm{~nm}$ ) so that it can propagate through the other fabrication layers and focusing it so that the irradiance becomes very strong at the point where the carrier generation is desired. Carriers may be generated at this focal point because of the square relationship for the irradiance of the TPA term in Equation 2.1.

\section{Research of Single-Event Effects in Photonics}

Radiation effects have been studied in the context of CMOS technology for decades, however, PICs have received limited treatment of radiation effect studies. Primarily, studies have been focused on TID effects in PICs. Some work has been accomplished in observing the behavior of photonic devices due to TID radiation exposure as in Bhandaru et. al. [3] Other research has been done in identifying the mechanisms that cause degradation of the performance of a MachZehnder modulator [4] and to model the effects caused by the TID [1]. A limited set of work has studied SEEs in PICs 
In the field of optics, beams of light have been used to modulate the transmissive properties of Si waveguides in a similar manner to ionizing radiation [23, 24]. Since light is itself a form of radiation, pulsed laser light is often used to emulate an ion strike in electronics [20] and may also be used in a similar manner for photonic devices. A recent paper by Goley et. al. [2] has demonstrated that SEEs may be of some significance to the operation of PICs in a space environment. This furthers the incentive to develop a comprehensive understanding of the mechanisms that may be involved in SEEs in PICs.

In the Goley paper [2], simulations were performed in the Lumerical Tool Suite for a waveguide that was given a profile of ion induced charge with an associated LET. For each charge profile, one optical simulation was performed by using the carrier densities of the profile as the input to a charge-to-index conversion model called the Soref and Bennett model [25] represented in Figure 6 [2]. The charge-to-index conversion model allows the simulation software to shift the refractive index of the $\mathrm{Si}$ in the waveguide in response to the charges induced by the single-event. Now that the perturbation of the waveguide's optical properties can be simulated, an optical simulation is used to compute the optical power losses and phase shifts seen at the output of the waveguide for each of the given charge profiles. A plot of the transmission loss versus LET with a trend curve is shown in Figure 7 [2].

The Soref and Bennett charge to index conversion model is represented in [25] by the equations

$$
\begin{aligned}
& \Delta n=\left(d n \_A p\right)(\Delta P)^{d n_{-} E p}+\left(d n_{-} A n\right)(\Delta N)^{d n_{-} E n} \\
& \Delta \alpha=\left(d \alpha_{-} A p\right)(\Delta P)^{d \alpha_{-} E p}+\left(d \alpha_{-} A n\right)(\Delta N)^{d \alpha_{-} E n}
\end{aligned}
$$

where $\Delta n$ and $\Delta \alpha$ represent the change in refractive index and optical absorption respectively from their non-excited values at a specified optical wavelength. The values $\Delta P$ and $\Delta N$ are the change 
in the Si's hole and electron concentrations in $\mathrm{cm}^{-3}$, and the values $d n_{-} A p, d n_{-} E p, d n_{-} A n, d n_{-} E n$, $d \alpha \_A p, d \alpha_{-} E p, d \alpha \_A n$, and $d \alpha_{-} E n$ is a set of coefficients that depend on the optical wavelength. The values of these coefficients for a wavelength of $1550 \mathrm{~nm}$ are given in Table 1 .

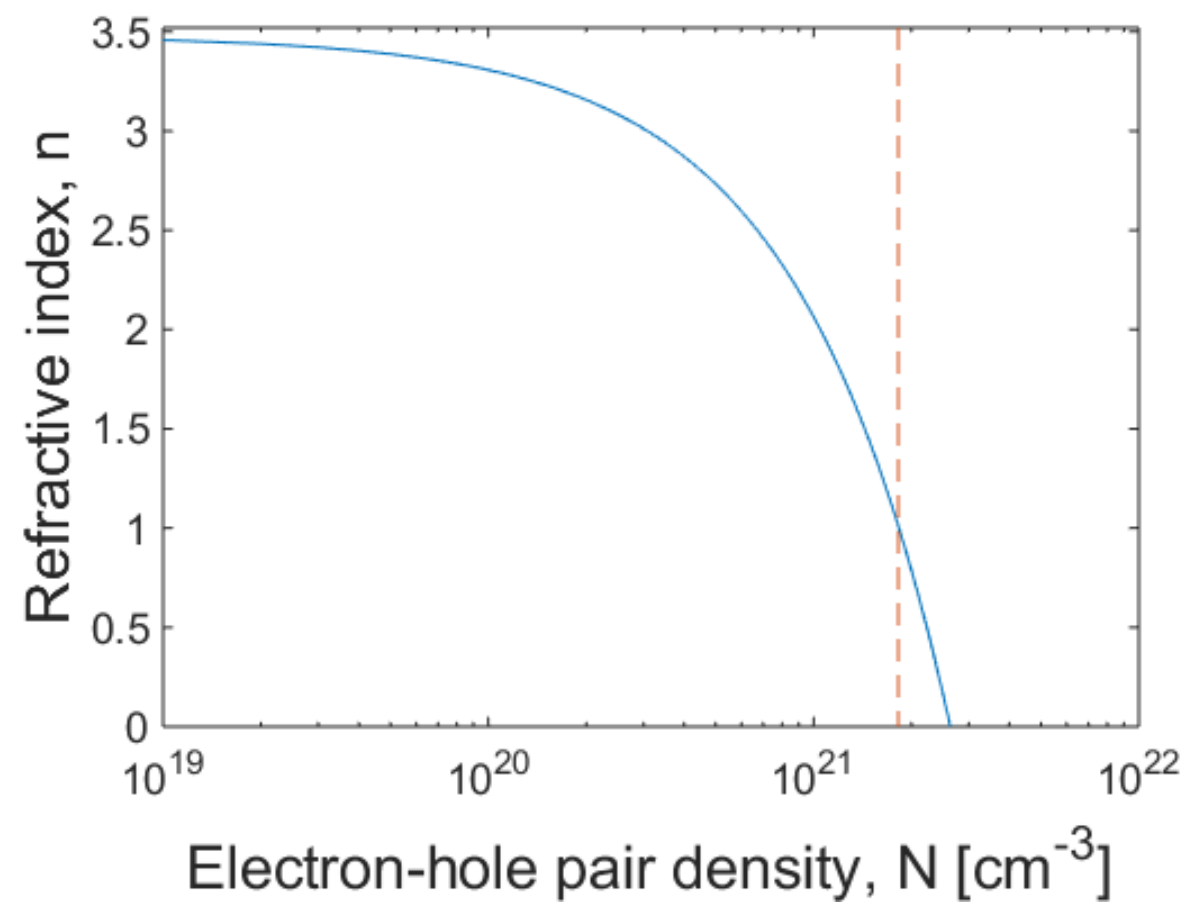

Figure 6

A graph of Si refractive index versus EHP density for the Soref and Bennet charge-to-index conversion model. As EHP density increases in Si, decreases in refractive index are observed. As the EHP density rises to about $1.83 \mathrm{e} 21 \mathrm{~cm}^{-3}$ the refractive index goes below 1 and starts to become reflective and absorbing like a metal [2] 


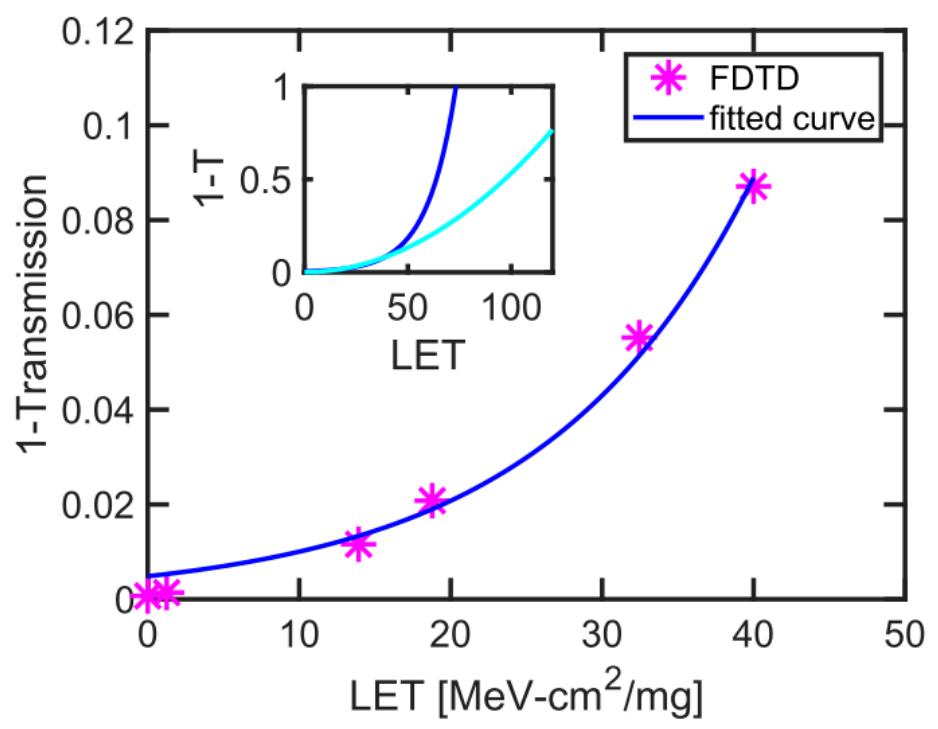

Figure 7

Trend of waveguide transmission losses versus LET with trendline from [2]. These values were computed in Lumerical FDTD for static charge profiles in a slab waveguide of cross-sectional dimensions of $220 \mathrm{~nm}$ wide by $450 \mathrm{~nm}$ high

Table 1

Coefficients of the Soref and Bennett charge-to-index conversion model for an optical wavelength of $1550 \mathrm{~nm}[25]$

\begin{tabular}{|c|c|}
\hline Coefficient & Value \\
\hline$d n \_A p$ & $-8.5 * 10^{-18}$ \\
\hline$d n \_E p$ & 0.8 \\
\hline$d n \_A n$ & $-8.8 * 10^{-22}$ \\
\hline$d n \_E n$ & 1 \\
\hline$d \alpha \_A p$ & $6 * 10^{-18}$ \\
\hline$d \alpha_{-} E p$ & 1 \\
\hline$d \alpha_{-} A n$ & $8.5 * 10^{-18}$ \\
\hline$d \alpha_{-} E n$ & 1 \\
\hline
\end{tabular}




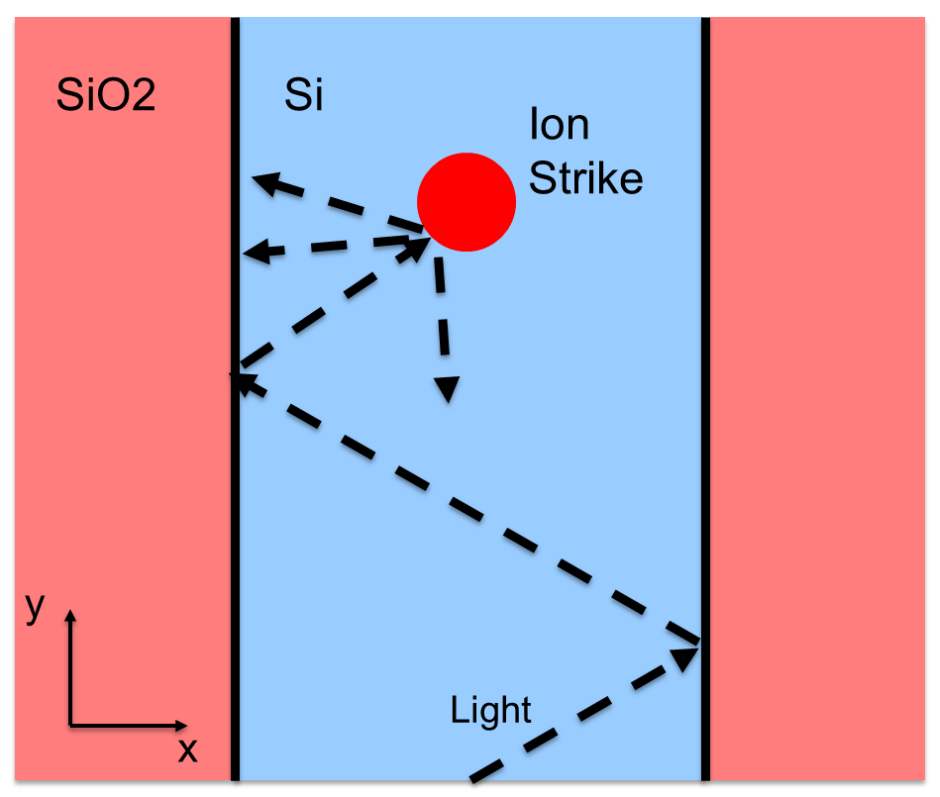

Figure 8

The mechanism of single-event radiation effects in a Si waveguide. Charges induced at the ion strike location cause shifts in the Si's refractive index. Significant free charges cause the Si to become reflective and absorbing

From these results Goley et al. predicted that transmission losses are the dominant effect of SEEs in Si waveguides with induced phase shifts being a secondary effect. They determined that the mechanism by which SEEs may affect photonic devices comes from the shift in the optical properties that are induced by the induced carriers. These induced carriers manifest as a cone of charge at the strike zone that shifts the index of the Si enough to create a metal-like material that both absorbs and reflects optical waves as illustrated in Figure 8.

These results are promising as they indicate the potential for limitations of Si PICs in space applications. However, there is still not a means of simulating the entire electro-optical effect in PICs for the duration that this SET takes place. The focus of this thesis is the development of such a simulation methodology in which a charge profile of arbitrary LET may be induced in an optical component and allowed to diffuse and recombine, and then allow for the simulation and extraction 
of transmission loss and phase shift for the full extent of that transient. This simulation methodology allows for the analysis of SEEs in PIC as well as the discovery of radiation hardness techniques in PICs.

\section{The Physics of Charge Transport Simulations}

At the core of any type of physics simulation, whether electrical, optical, or some other physical domain, is a set of equations that represents the behavior of the physical objects and quantities being observed and their interactions with other objects and quantities of interest. With these equations, various physical situations may be setup in software and tested to reveal how a device may respond to various stimuli in ways that cannot be accomplished by traditional experimentation. In this way, new discoveries about internal device physics may be made via simulation if they can also be verified with more surface level experiments.

Before any computation can occur, a charge transport solver must first break a device down into a set of finite elements (called a mesh) since a computer cannot compute infinitesimally small elements. The finer the mesh is, the more representative the simulation will be of a true device, but at the cost of computational resources. Once a device mesh has been calculated, a charge transport simulation at a minimum requires the computation of the drift-diffusion equations, Poisson's equation, and the continuity equation. Poisson's equation is an application of Gauss' law

$$
\nabla \boldsymbol{E}=\frac{\rho}{\epsilon_{0}}
$$

where $\boldsymbol{E}=-\nabla \phi$ and $\rho=q\left(p-n+N_{d}-N_{a}\right)$. If these substitutions are made in Gauss' law then Poisson's equation is obtained: 


$$
\nabla^{2} \phi=-\frac{q}{\epsilon_{0}}\left(p-n+N_{d}-N_{a}\right) .
$$

In Equation 2.5, $\phi$ is the electrostatic potential, $\epsilon_{0}$ is vacuum permittivity, $q$ is the charge of an electron, $p$ is hole density, $n$ is electron density, $N_{d}$ is donor density, and $N_{a}$ is acceptor density. Poisson's equation relates the electrostatic potential (and thus the electric field) to the local charge density. This equation allows the charge transport solver to obtain the electric field which is required to solve the drift-diffusion equations.

The drift-diffusion equations are given by

$$
\begin{aligned}
& \mathbf{J}_{\mathbf{n}}=q \mu_{n} n \boldsymbol{E}+q D_{n} \nabla n \\
& \mathbf{J}_{\boldsymbol{p}}=q \mu_{p} p \boldsymbol{E}-q D_{p} \nabla \mathbf{p}
\end{aligned}
$$

where $\mathbf{J}_{\boldsymbol{n}}$ and $\mathbf{J}_{\boldsymbol{p}}$ are the electron and hole current density vectors respectively, $\mu_{n}$ and $\mu_{p}$ are the material electron and hole mobilities respectively, $D_{n}$ and $D_{p}$ are the electron and hole diffusivities respectively, and $\boldsymbol{E}$ is the electric field vector. The diffusivities are given by Einstein's relation

$$
D_{n, p}=\mu_{n, p} \frac{k_{B} T}{q}
$$

where $k_{B}$ is Boltzmann's constant and, $\mathrm{T}$ is the temperature in kelvin. The drift-diffusion equations can be interpreted as taking the profiles of electron and hole densities in a device and the electric field inside the device as inputs and generating the vector field of current density as the output. This current density vector field represents the flow of the charges throughout the device being simulated.

Finally, the continuity equations are given by

$$
\begin{aligned}
& \frac{\delta \mathrm{n}}{\delta t}=\frac{1}{q} \nabla \cdot \mathbf{J}_{\mathbf{n}}+G_{n}-R_{n} \\
& \frac{\delta \mathrm{p}}{\delta t}=-\frac{1}{q} \nabla \cdot \mathbf{J}_{p}+G_{p}-R_{p}
\end{aligned}
$$


where $R_{n}$ and $R_{p}$ are the electron and hole recombination rates respectively, and $G_{n}$ and $G_{p}$ are the electron and hole generation rates respectively. These equations relate the current density fields and recombination rates to the change in electron and hole densities over time. Prior to the computation of the continuity equations, the contribution of the various types of recombination rates (Shockley-Read-Hall, Auger, radiative, etc.) may be calculated from the current state of the charge state of the device and the electric field. After the continuity equations are solved, the change in current densities $(\delta n / \delta t$ and $\delta p / \delta t)$ are multiplied by a time step determined by the solver to obtain a total change in charge density that is added to the previous charge profiles.

$$
\begin{aligned}
& n(t+\Delta t)=n(t)+\frac{\delta n}{\delta t} \Delta t \\
& p(t+\Delta t)=p(t)+\frac{\delta p}{\delta t} \Delta t
\end{aligned}
$$

These new charge profiles are then used as the input to the next iteration of Poisson's equation and the process may be repeated until the simulator no longer requires any new time points.

\section{The Physics of Optical Simulations}

The most common method of computation for 3D optical simulations is called the finitedifference time-domain (FDTD) method. This method computes Maxwell's equations of electric and magnetic fields by discretizing the problem in both space and time. Maxwell's equations for non-magnetic materials are given in [26] as

$$
\begin{aligned}
& \nabla \cdot \boldsymbol{E}=\frac{\rho}{\epsilon_{r} \epsilon_{0}} \\
& \nabla \cdot \boldsymbol{H}=0 \\
& \nabla \times \boldsymbol{E}=-\mu_{0} \frac{\delta \boldsymbol{H}}{\delta t} \\
& \nabla \times \boldsymbol{H}=\boldsymbol{J}+\epsilon_{r} \epsilon_{0} \frac{\delta \boldsymbol{E}}{\delta t}
\end{aligned}
$$


where $\epsilon_{0}$ is the permittivity of free space, $\epsilon_{r}$ is relative permittivity, $\mu_{0}$ is the permeability of free space, $\rho$ is local charge density, $\boldsymbol{J}$ is current density, $\boldsymbol{E}$ is the electric field, and $\boldsymbol{H}$ is the magnetic field. All optical devices and effects in may be sufficiently simulated with these equations. In an FDTD solver, the derivatives in these equations are replaced with finite differences to facilitate computation.

Due to the relation between a material's refractive index and its permittivity and permeability the shifts in refractive index from the Soref and Bennett model (Equations 2.2 and 2.3) may be captured as shifts in relative permittivity.

$$
\epsilon_{r}=n^{2} \text { (for non-magnetic material) }
$$

\section{Chapter Conclusions}

The fundamental component of PICs is the waveguide. Basic waveguide transmission theory shows that optical waves may be confined to a waveguide through the law of total internal reflection. The confinement of the optical waves in the waveguide gives rise to various modes of oscillation that depend heavily on the waveguide dimensions and carrier wavelength. Some of these modes may be used to carry information, however, the fundamental mode is the most commonly used as it is the most convenient and efficient mode to excite.

There are several different radiation sources that are encountered by space systems. One source being the Van Allen belts that are belts of particles trapped in the magnetic field around the earth. This poses a threat to satellites orbiting the earth as well as systems leaving the earth that must pass through the belts. Solar activity is another radiation source which includes solar flares where the sun may eject large volumes of heavy ions into the space environment of electronic 
systems. The final primary radiation source is GCRs that are highly energetic rays that come from outside the solar system.

SEEs are the effects in electronic systems caused by the interaction of a single high energy particle with a device in the system. If a heavy ion strikes a semiconductor junction, energy is deposited onto the $\mathrm{Si}$ and free carriers are generated as a result. These free carriers will be swept away be the junction's depletion region and manifest as a current transient in the nodes attached to the junction. This transient may cause voltage fluctuations, data corruption, or even permanent device damage.

Little has been done to research SEEs in PICs. What research has been done has shown that potential limitations may exist in PICs due to SEEs because induced charges in Si waveguides cause shifts in the refractive index and absorption of the Si. This leads to motivation to create a simulation methodology where the full transient interaction of any arbitrary optical device with a heavy ion may be simulated. The focus of this thesis is the development of such a simulation methodology that aides in the analysis of these SEEs in PICs and the discovery of radiation hardness techniques. 


\section{CHAPTER III}

\section{ELECTRO-OPTICAL SIMULATION METHODOLOGY}

This chapter provides an in-depth walkthrough of the use of the proposed simulation methodology using the example of a slab waveguide with cross-sectional dimensions of $200 \mathrm{~nm}$ by $700 \mathrm{~nm}$. First the simulation tools used in this work are introduced and followed by an overview of the methodology. The methods and assumptions are detailed and then each of the three stages of this methodology are broken down to explain their inner workings.

\section{Simulation Tools}

The simulation tools used in this thesis are provided in the Lumerical Tool Suite [27] for electrical and optical simulations. The Lumerical Tool Suite contains four simulation tools, however only two of these are used here: one electrical charge transport solver, and one optical finite-element optical solver. Lumerical's charge transport solver is called DEVICE and it allows for simulating the charge generation, diffusion, and recombination that is required for SEE simulation. Lumerical's finite-element optical solver is called FDTD (short for Finite Difference Time Domain) which allows an optical device's parameters like transmission loss and phase shift to be extracted for analysis. A feature of FDTD that is important to this thesis is the feature of importing charge information from DEVICE simulations and allowing it to perturb the refractive index of the simulated device. 


\section{Methodology Overview}

Compared to the simulation of SEEs in electronic devices, photonic devices have a certain set of special requirements. In CMOS and other electronic technologies, the post-ion strike effects exist predominantly in the electronic domain, and thus, finite-element simulation will likely be constrained to a charge transport solver. However, SEEs in devices that facilitate the transmission and manipulation of optical signals require that simulation be carried out in both the electronic and optical domains due to needing to simulate 1) the effect of an ion striking the device, 2) the effects of the charge generation, diffusion, and recombination in response the ion strike, and 3) the effect of the induced charges shifting the material's optical properties. These effects exist in separate domains (the optical and electrical domains) yet they are interdependent on each other through effects that couple the electrical and optical domains such as the optical generation of free charges and the perturbation of refractive index due to the free charges. The co-simulation of the electrical and optical domains is the biggest challenge associated with developing a comprehensive method for simulating SEEs in PICs. This challenge is overcome by breaking the simulation of photonic SEEs into three separate stages (flowchart illustrated in Figure 9). This includes simulations of (A) energy transferred during an ion strike, (B) charge generation, and (C) the optical response to transient charge generation.

The methodology starts with step A in Figure 9 with a simulation that emulates the effect of the ion strike on the device in the optical domain by injecting a Gaussian laser light source into the device at the desired strike location and angle. With this simulation, a 3D profile of power absorption per unit volume is created that corresponds to the generation rate of electrons and holes at each point in the device. For each strike location and angle the user wishes to simulate, it will 
be necessary to perform one of these simulations to generate a unique power absorption profile corresponding to that location and angle.

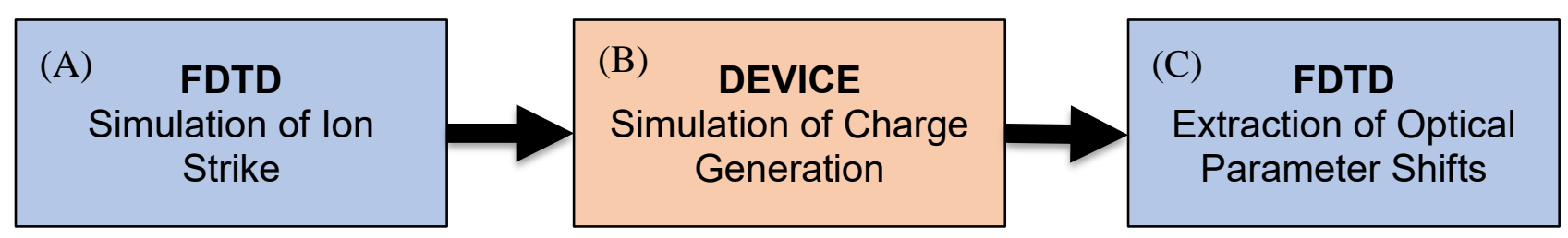

Figure 9

Flowchart of the proposed simulation methodology that makes it possible to simulate the full time-domain effect of an ion strike in any arbitrary optical device. In A) an optical domain simulation is performed to emulate the effect of an ion striking the device. A power absorption profile is computed, and this is passed to stage B) where an impulse of charges is generated, diffused, and recombined to capture the electronic effects of the ion strike. A set of discrete-time 3D charge profiles are then generated, and these are passed to stage $\mathrm{C}$ ) where each profile is used to compute the shifts in optical phase and power loss that represent the overall device response to the ion strike. While this figure shows Lumerical FDTD and DEVICE being used for the optical and electrical domains respectively, this methodology should generally work with any optical and electrical simulation tools with similar features

Once power absorption profiles are obtained, they are passed to the simulation represented by step B in Figure 9, where the profiles are converted to charge generation profiles used to excite an impulse of charges in the device and simulate their diffusion and recombination. At this point in the simulation methodology, the LET of the simulated ion strike may be estimated by the using the software's total charge integration algorithm and using the formula

$$
L E T=\frac{N_{t} E_{g}}{10^{6} \rho l}
$$

where LET is the total LET in $\mathrm{MeV}-\mathrm{cm}^{2} / \mathrm{mg}, N_{t}$ is the total number of carriers generated in the device, $E_{g}$ is the bandgap in the semiconductor $(1.1 \mathrm{eV}$ in $\mathrm{Si}), \rho$ is the density of the semiconductor material $\left(2650 \mathrm{mg} / \mathrm{cm}^{3}\right.$ for $\left.\mathrm{Si}\right)$, and $l$ is the path length of the ion through the device. When LET 
is estimated by this formula, it may be tuned in the software by adjusting the intensity of the generation source profile. The simulation time should be set such that it allows all or most EHPs to recombine. For active devices that have contacts connected to electronic circuits this may be as short as a few nanoseconds. For passive devices with no contacts, simulations have shown this time to be on the order of tens of microseconds. The output of this charge transport simulation is a set of discrete time charge profiles that represents the changing charge state inside the device at different times during the event.

Each of these charge profiles (or more likely a subset of them) are used in the final stage in step C in Figure 9, where they are used to shift the device's refractive index and absorption via the Soref \& Bennett charge-to-index conversion model. This final optical domain simulation sweeps through the charge profiles and captures a set of s-parameters that represent the optical input/output response of the device at the same point in time that is represented by the charge profile. This allows the extraction of a set of time-dependent s-parameters from which the timedomain transient phase shift and transmission losses of the device may be extracted. These phase shifts and transmission losses represent the response of the optical device to the ion strike and are thus the final results of the simulation methodology.

While Figure 9 uses the example of the Lumerical tool suite (FDTD and DEVICE) that this simulation work was performed in, this simulation methodology should be compatible with any simulation tools of a similar feature set. One example of a software substitution that may be compatible with this methodology would be substituting Lumerical DEVICE with Synopsys Sentaurus [28], which may be used in conjunction with Lumerical FDTD. Unlike Lumerical DEVICE, Sentaurus natively includes radiation effects tools that could prove to be beneficial to research performed with this methodology. 


\section{Methods}

The waveguide evaluated in this paper is a Si slab waveguide shown in Figure 10. The cross-sectional dimensions are $700 \mathrm{~nm}$ by $220 \mathrm{~nm}$. This type of waveguide is typical for component-to-component transmission applications. The waveguide operates under the fundamental TE mode (shown in Figure 11) and is designed to operate with a carrier wavelength of $1550 \mathrm{~nm}$. So that each stage of the methodology remains consistent, the same device dimensions and materials must be entered into each simulator (FDTD and DEVICE) identically. Failure to do so may result in a mismatch in the dimensions of the power absorption profiles and the device dimensions in the charge transport simulation, and/or a mismatch in the dimensions of the charge profiles and the dimensions of the device in the final s-parameter extraction simulation. This mismatch will likely cause either a simulation divergence or erroneous simulation results.

A Gaussian beam with a wavelength of $500 \mathrm{~nm}$ and diameter of $50 \mathrm{~nm}$ is used to emulate the ion striking the waveguide. In Si, a wavelength of $500 \mathrm{~nm}$ causes SPA to occur since its photon energy is above the bandgap in $\mathrm{Si}(1.12 \mathrm{eV})$ [19]. SPA is often used experimentally to interrogate the detailed spatial dependence on the SEE vulnerability, not possible with broadbeam heavy ion irradiation. In SPA testing, the photon energy is greater than the semiconductor bandgap, thus a photon with a wavelength of less than $1.1 \mu \mathrm{m}$ may be used to generate a single electron-hole pair (EHP) [14]. In this thesis, the simulated ion strikes are assumed to be striking the waveguide normally from the upper surface (as shown in Figure 12) and alternate strike angles are not considered here. 


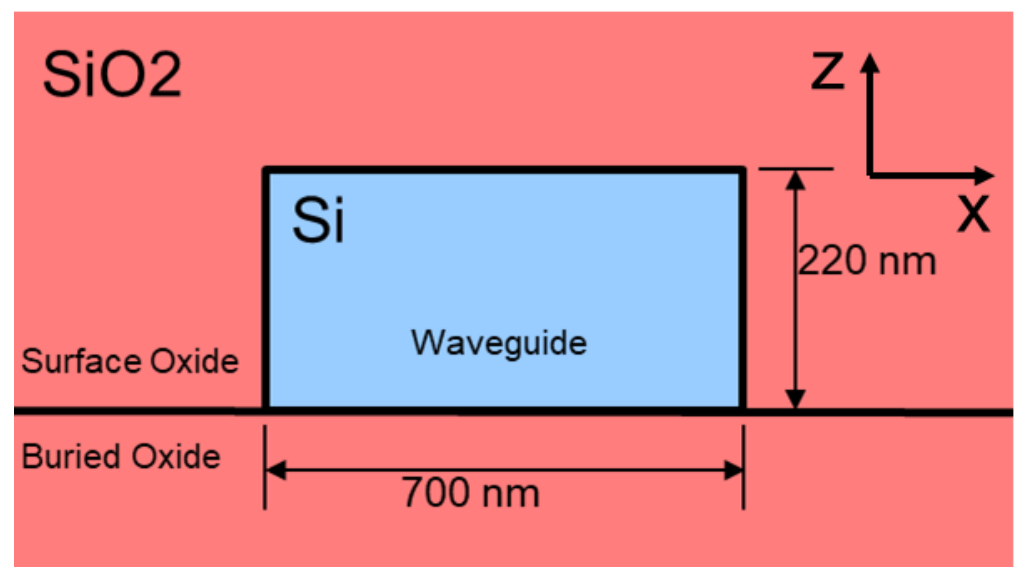

Figure 10

A cross-sectional slice of the simulated Si slab waveguide. The waveguide is $700 \mathrm{~nm}$ wide by $220 \mathrm{~nm}$ high. This $\mathrm{Si}$ slab is surrounded entirely by $\mathrm{SiO} 2$ to create the reflective barrier required for guiding the light waves. The $\mathrm{SiO} 2$ is split into separate a separate surface oxide and buried oxide

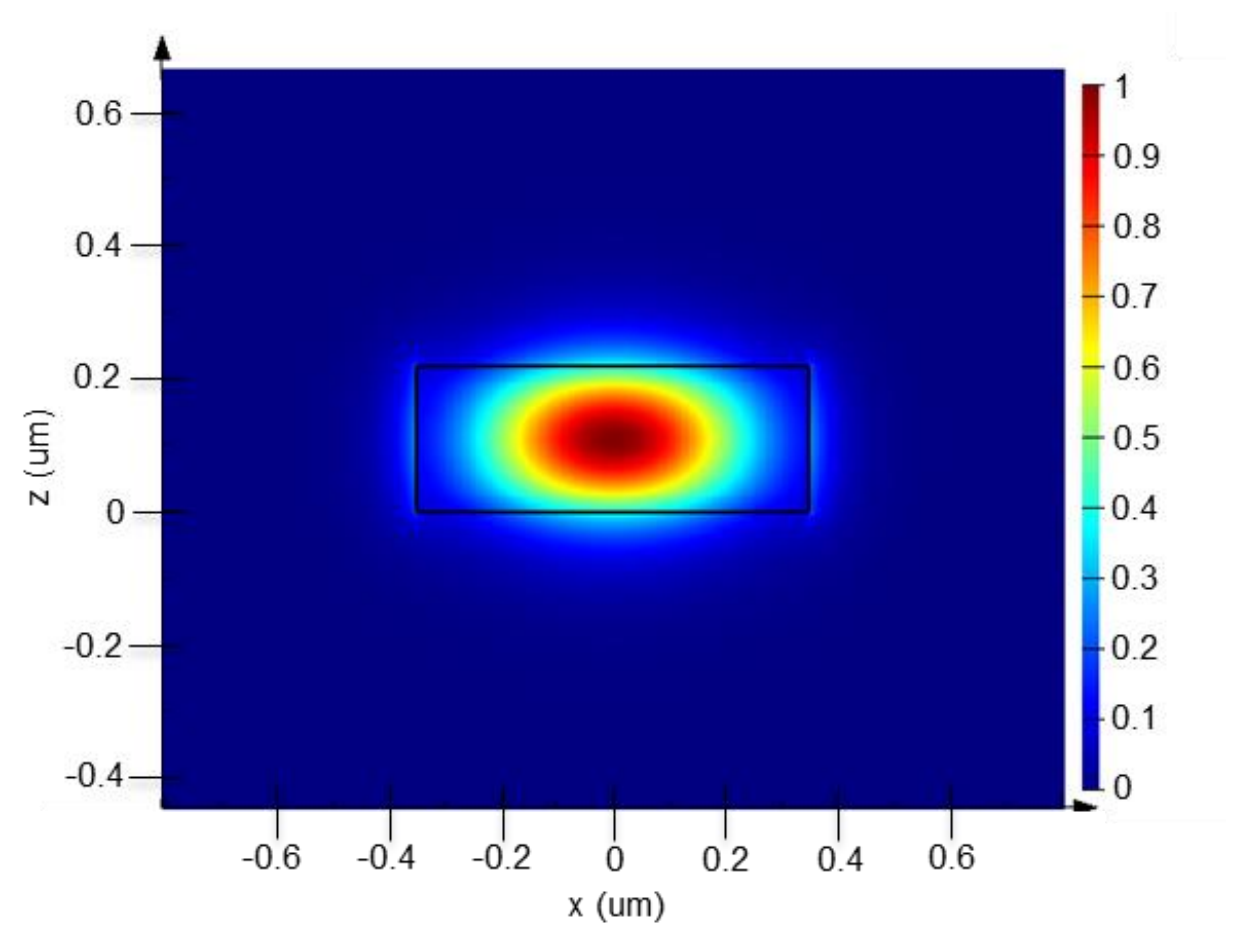

Figure 11

The normalized electric field intensity of the fundamental TE mode of the simulated waveguide shown in Figure 10 


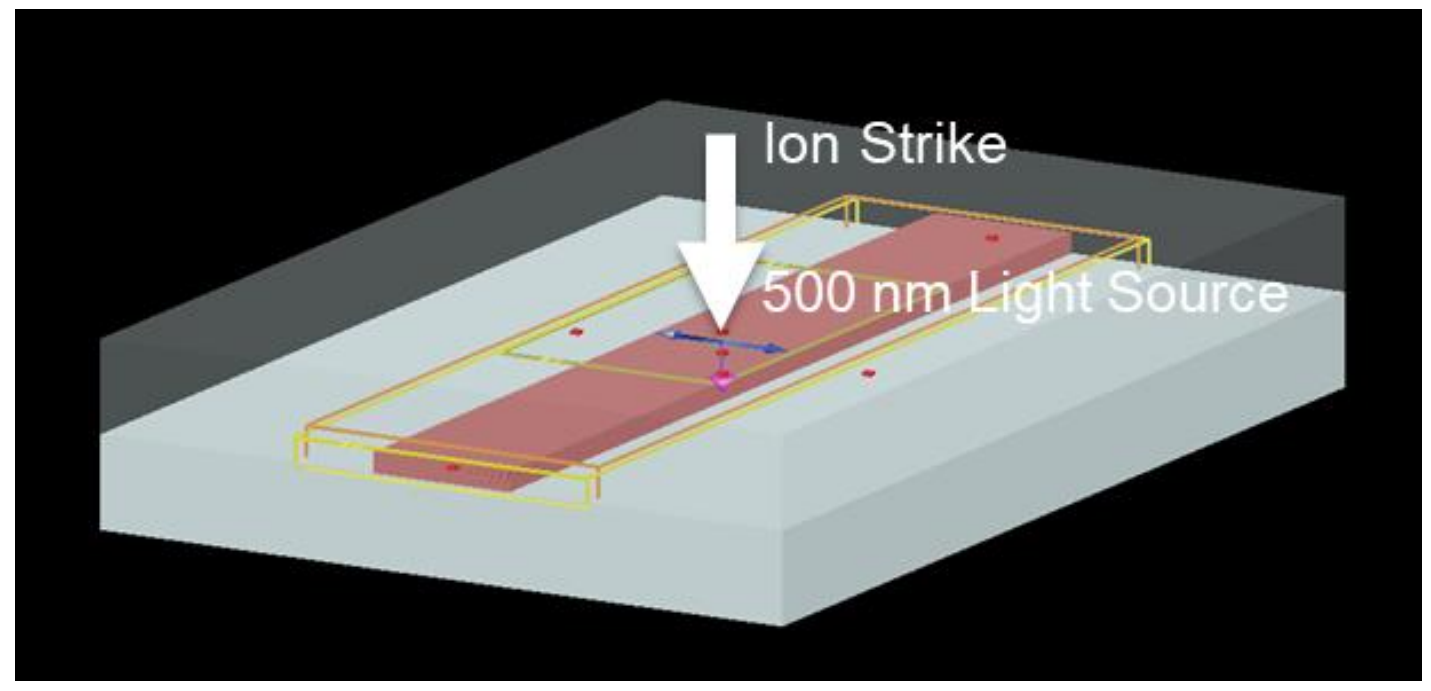

Figure 12

The 3D model of the Si waveguide in Lumerical FDTD. The white arrow represents the location and direction of the simulated ion strike using SPA

For each simulation (both optical and electrical), mesh constraints were added that generate a finer mesh in the region directly surrounding the ion strike (as shown in Figure 13). This is necessary to attain accurate simulation results and to prevent the divergence of the simulations caused by fast changing gradients in the charge density at the location of the ion strike. In general, having a mesh edge length that is longer than radius of the ion strikes charge generation profile (in this case about $25 \mathrm{~nm}$ ) is not sufficient to capture the details of the ion strike in the simulation. For both the optical and charge transport simulations in this thesis, the max mesh length has been set to $5 \mathrm{~nm}$ in the region surrounding the ion strike location so that a sufficiently fine mesh may be generated. 


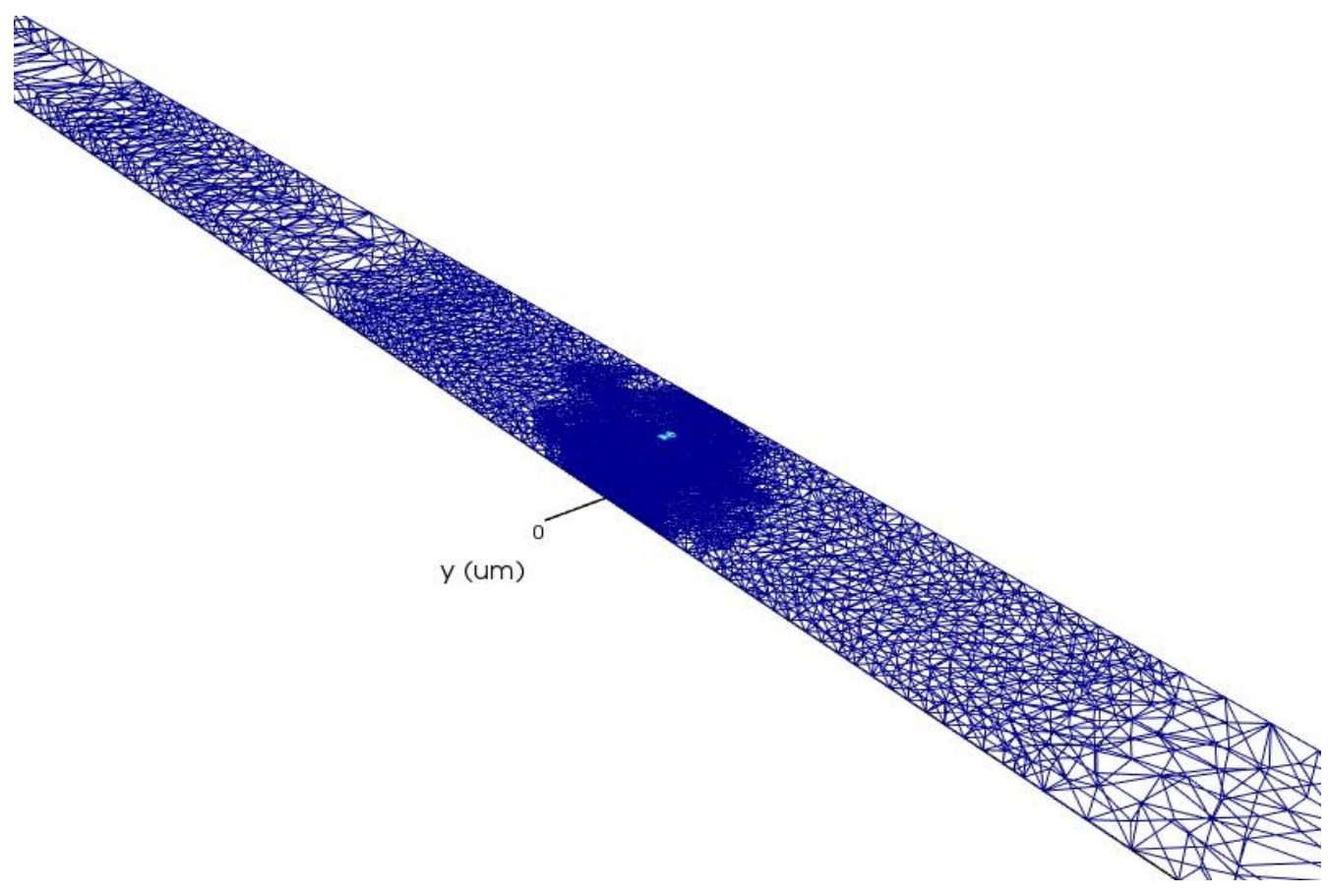

Figure 13

The generated mesh surrounding the ion strike location in Lumerical DEVICE. The mesh gets finer in the regions closer to the center of the strike location to prevent fast changing spatial gradients in the charge density from causes the simulation to diverge

\section{Stage A - Emulation of Heavy Ion Strike in Optical Domain}

The goal of the first simulation step (step A in Figure 9) in this methodology is to generate a power absorption profile that represents the charge deposition rate of an ion striking the optical device. The mechanism by which this happens is nearly identical in result to the mechanisms of the photoelectric effect, and thus lasers may be injected into devices to emulate and ion strike [19, 20]. These tools are leveraged in this methodology to emulate the effect of an ion striking a photonic device. The output of this simulation is a 3D power absorption profile that represents the EHP generation rate at each point in the simulated device. 


\section{Device Setup}

The device dimensions and materials were constructed in the solver using the software's built in shapes. In Lumerical, when a new shape or simulation tool is added to the simulation environment, it is put into the "Objects Tree". The objects tree that is necessary for this first simulation is shown in Figure 14.

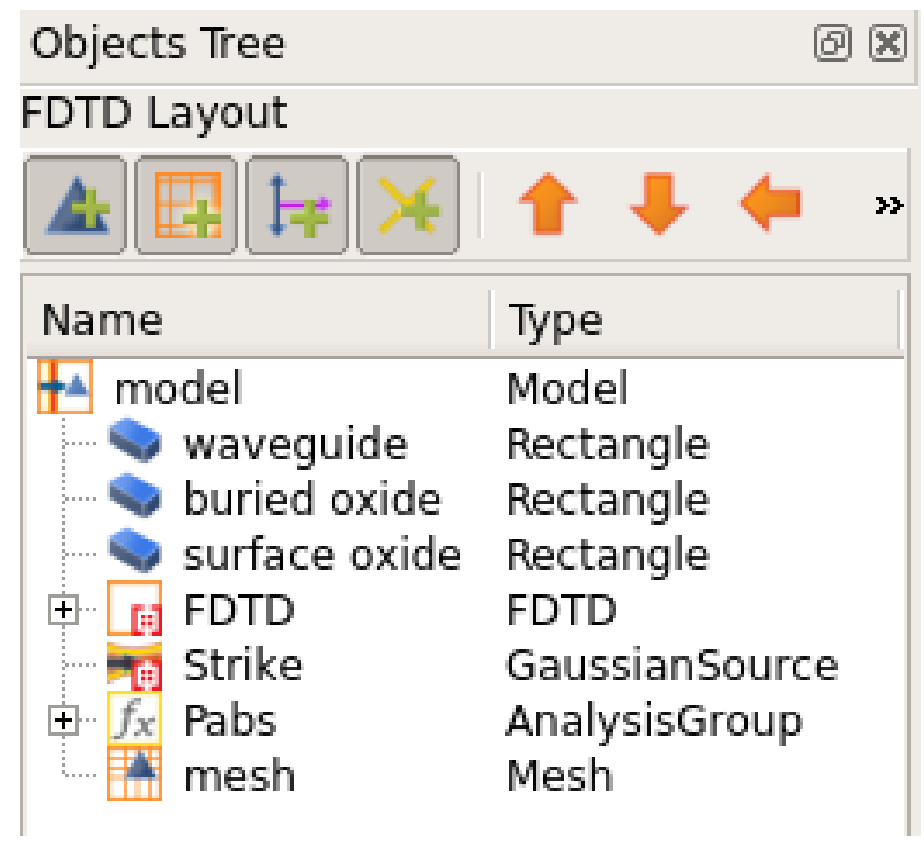

Figure 14

The object tree in Lumerical FDTD with the tools required for the ion strike simulation. The objects named "waveguide", "buried oxide", and "surface oxide" are the shapes used to define the dimensions and materials of the waveguide in this example. "FDTD" is the FDTD solver object that defines the simulation region and mesh and the object called "mesh" is the mesh override object that is defining a finer mesh directly surrounding the ion strike location. Unique to this simulation is the Gaussian light source (called "Strike") and the power absorption monitor (called "Pabs")

In Figure 14, the device dimensions and materials are defined by the object's "waveguide", "buried oxide", and "surface oxide". The "waveguide" object represents the waveguide's Si slab 
(that is the material that the light propagates through represented in blue in Figure 10). The "buried oxide" and "surface oxide" objects represent the $\mathrm{SiO} 2$ material encapsulating the waveguide slab (represented in red in Figure 10) that establishes the reflective barrier for the waveguide. Separating the device's oxide into the "buried oxide" and "surface oxide" is not strictly necessary; however it may make it straightforward to define different materials for the surface and buried oxides if it is desired. The geometries of each these objects are set by right-clicking them and choosing "properties" from the drop-down menu. For this example, the waveguides crosssectional dimensions are set to the dimensions shown in Figure 10.

\section{Simulation Region Setup}

An FDTD solver simulation region must also be defined (represented in Figure 14 by "FDTD"). This region is generally made to be slightly smaller than the region of $\mathrm{SiO} 2$ represented by the surface oxide and buried oxide to prevent any part of the simulation region from not having any materials defined for it. For this simulation, the waveguide does not have to be set to be particularly long along the length of the waveguide (the y dimension) since this is not a simulation measuring typical light propagation through the waveguide. The Si's high absorption of the 500 $\mathrm{nm}$ wavelength light prevents the light from travelling very far along the y direction. Therefore, to save on computation time, the simulation region is defined to only be $5 \mu \mathrm{m}$ long. The full dimensions have been defined as $1.5 \mu \mathrm{m} \times 5 \mu \mathrm{m} \times 0.44 \mu \mathrm{m}$ for this example as shown in Figure 15.

The default meshing scheme is set up within the properties of the FDTD solver object as shown in Figure 16. The default mesh in the $\mathrm{x}$ and $\mathrm{y}$ directions is set to be defined by a "maximum mesh step" of $20 \mathrm{~nm}$. The mesh in the z direction has been specially defined in this case to be 
defined by 10 "mesh cells per wavelength". The reason for defining it this way is due to the fact that the ion strike is occurring along the $\mathrm{z}$ axis in this example and defining by "mesh cells per wavelength" automatically changes the meshing resolution in the $\mathrm{z}$ direction if a different wavelength was chosen to represent the emulated ion strike. The "minimum mesh step" setting has been set to $5 \mathrm{~nm}$ which is an order of magnitude smaller than the diameter of the light source emulating the ion strike $(50 \mathrm{~nm})$. The remainder of the mesh settings in Figure 16 are set to their default values.

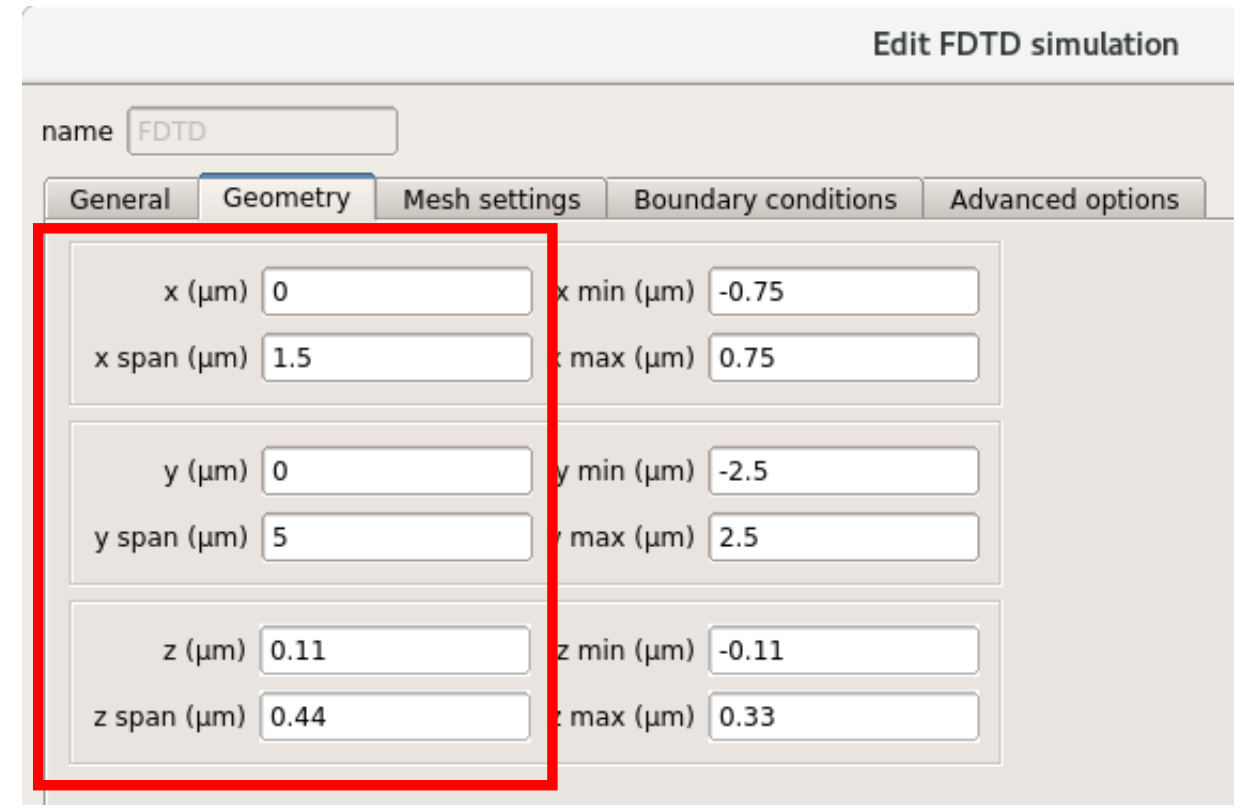

Figure 15

Defined dimensions of the FDTD simulation region for the ion strike simulation. This region encapsulates the waveguide of Figure 10. The simulation region has been defined as $1.5 \times 5 \times$ $0.44 \mu \mathrm{m}^{3}$ 


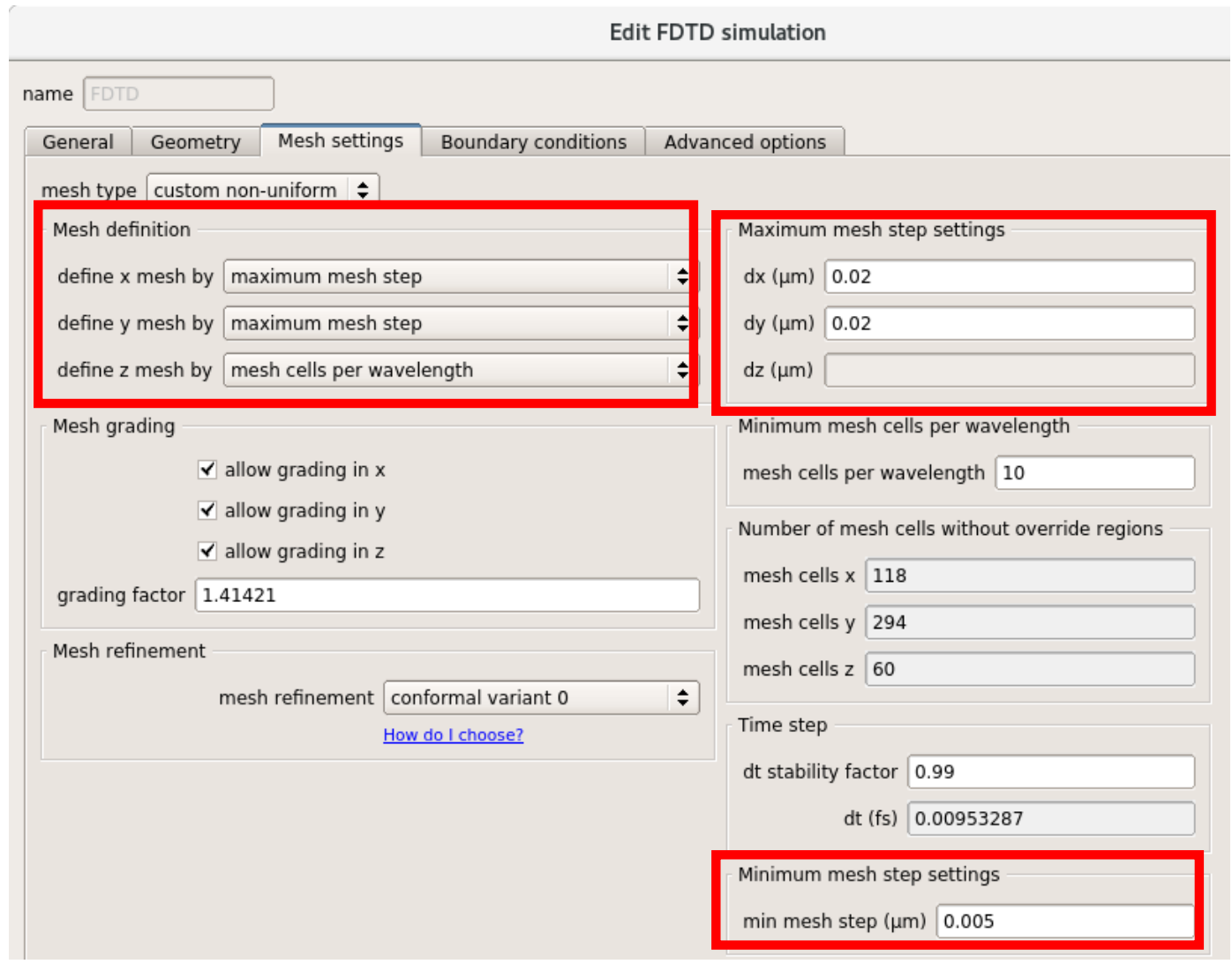

Figure 16

The mesh settings of the ion strike simulation. The maximum mesh step is set to $20 \mathrm{~nm}$ and the minimum mesh step is set to $5 \mathrm{~nm}$. The mesh in the $\mathrm{z}$ direction has been set to be defined by 10 "mesh cells per wavelength" instead of by "maximum mesh step" since the ion strike is propagating in the $\mathrm{z}$ direction for this example 


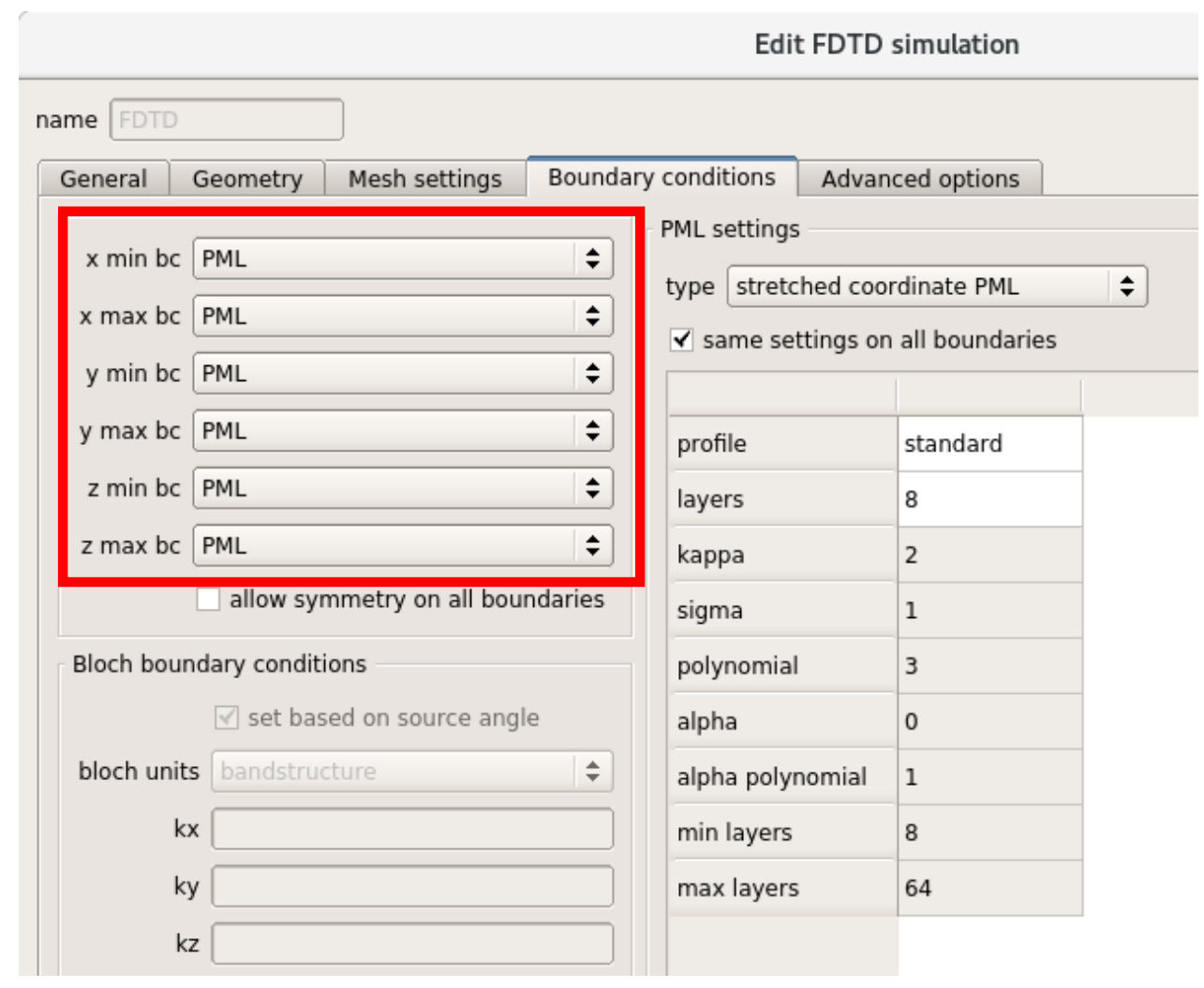

Figure 17

The boundary condition settings in Lumerical FDTD. The boundary condition at each boundary ("x min bc", "x max bx", "y min bc", etc.) is set to perfectly matched layer (PML). The PML settings are set to their defaults

At the edges of the simulation region are the simulation boundaries. So that the reaction of light as it hits the simulation boundary may be defined, boundary conditions must be set as in Figure 17. For these simulations, the boundary conditions are all set to perfectly matched layer (PML) as this allows the light to react to the simulation boundary as though the material at the boundary is extended to a theoretical infinity. This allows light to pass through the boundaries where $\mathrm{Si}$ is present and allows the simulator to act as though it is simulating a theoretically infinitely long waveguide. 


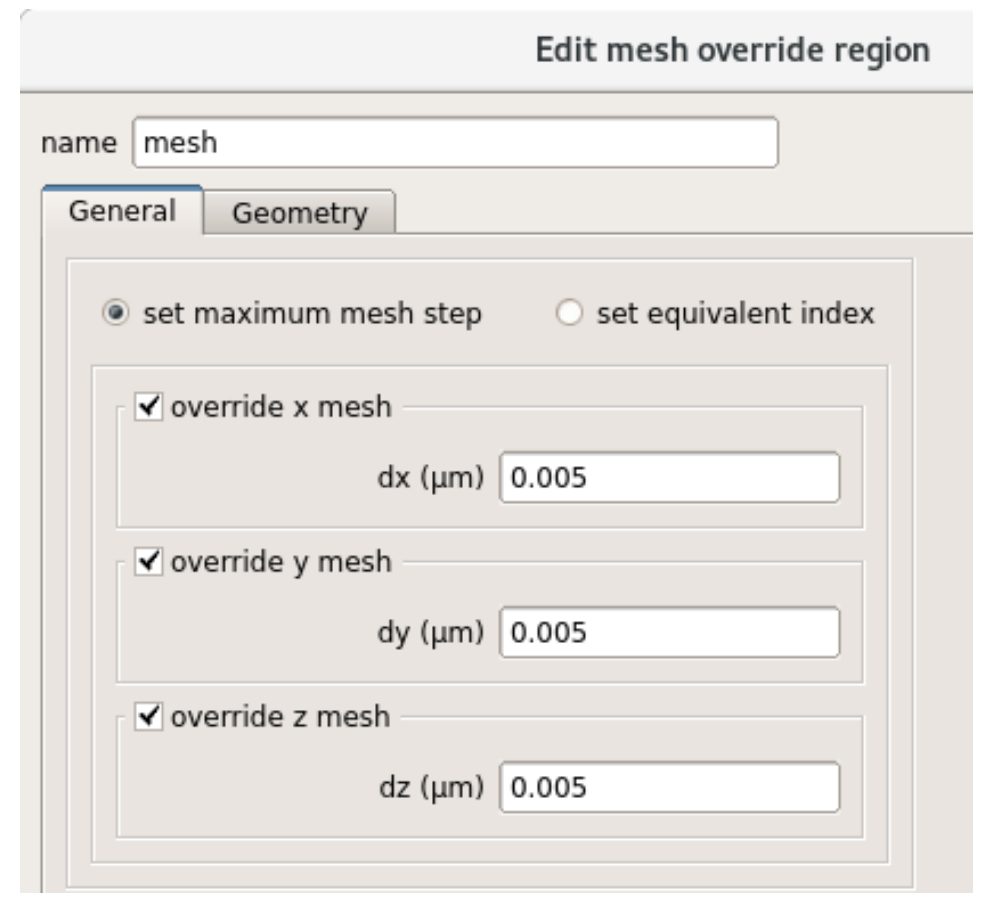

Figure 18

Mesh override settings for the region directly surrounding the ion strike. This defines the maximum mesh step size to be $5 \mathrm{~nm}$ along all axes. This guarantees that the generated power absorption profile will have a sufficiently fine resolution

A mesh override region is also defined directly around the location of the ion strike in the device. This mesh override region has been set to a maximum mesh size of $5 \mathrm{~nm}$ along all axes to guarantee that the mesh is sufficiently fine to capture any details in the region of the ion strike. The dimensions of this mesh override region span the height of the waveguide along the $\mathrm{z}$ direction $(0 \mathrm{~nm}<\mathrm{z}<220 \mathrm{~nm})$ and a $250 \mathrm{~nm}$ by $250 \mathrm{~nm}$ square centered around the region of the ion strike location as shown in Figure 19. 


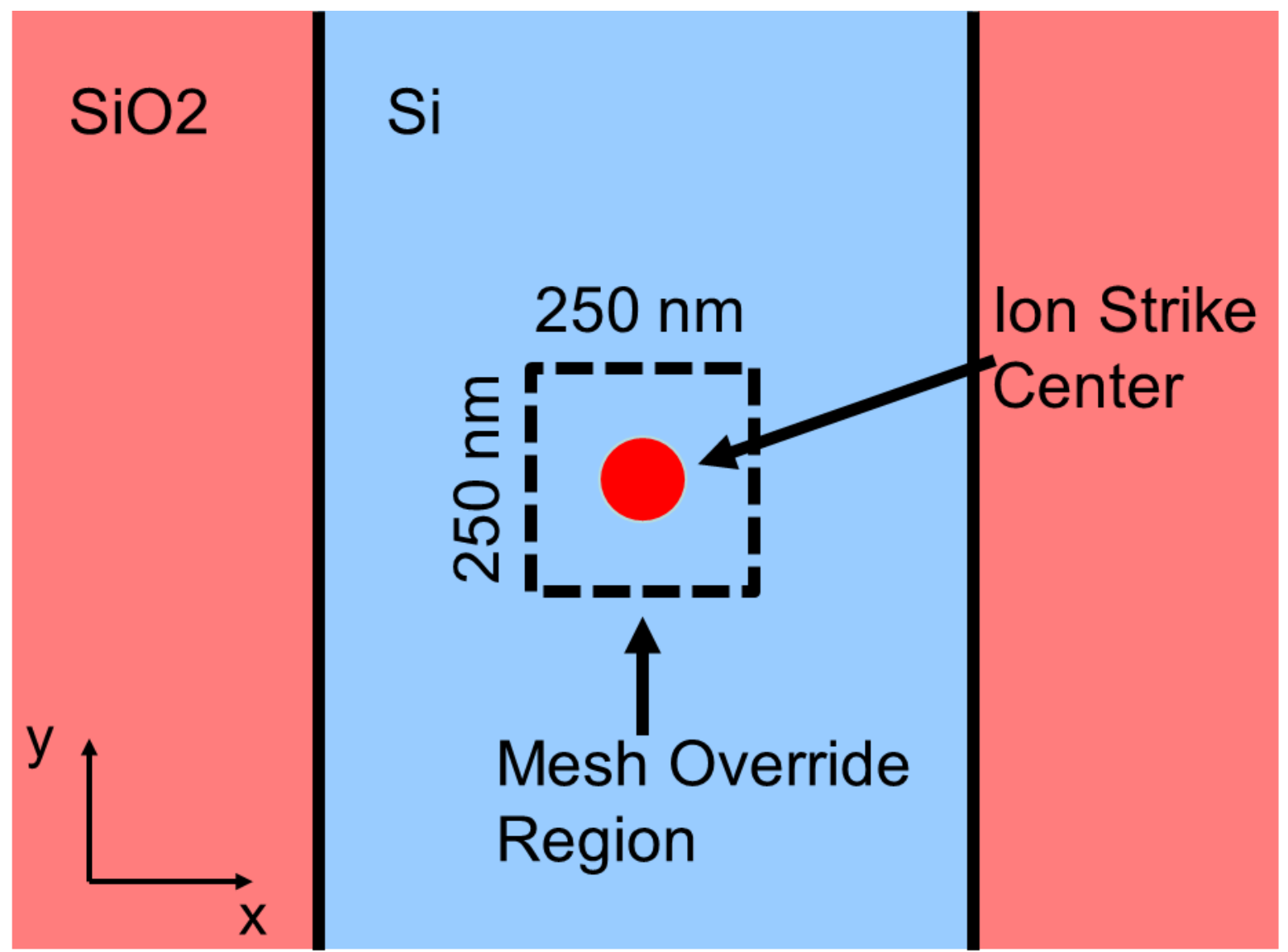

Figure 19

The location and dimensions of the mesh override region

\section{Gaussian Light Source}

A Gaussian light source object has been placed directly on top of the waveguide (a $\mathrm{z}$ coordinate of $220 \mathrm{~nm}$ as shown in Figure 20) and set to "z-normal" so that it injects light downward along the $\mathrm{z}$ axis. This Gaussian source is set to have a "x span" and "y span" of $50 \mathrm{~nm}$ to represent an ion strike with a diameter of about $50 \mathrm{~nm}$. In Figure 21 the wavelength of this source has been defined as $500 \mathrm{~nm}$ by selecting “override global source settings" and "set frequency/wavelength", 
and then defining "wavelength start" and "wavelength stop" to both be $500 \mathrm{~nm}$. This ensures that the source wavelength will be as close to a pure $500 \mathrm{~nm}$ wave pulse as is possible for the simulator.

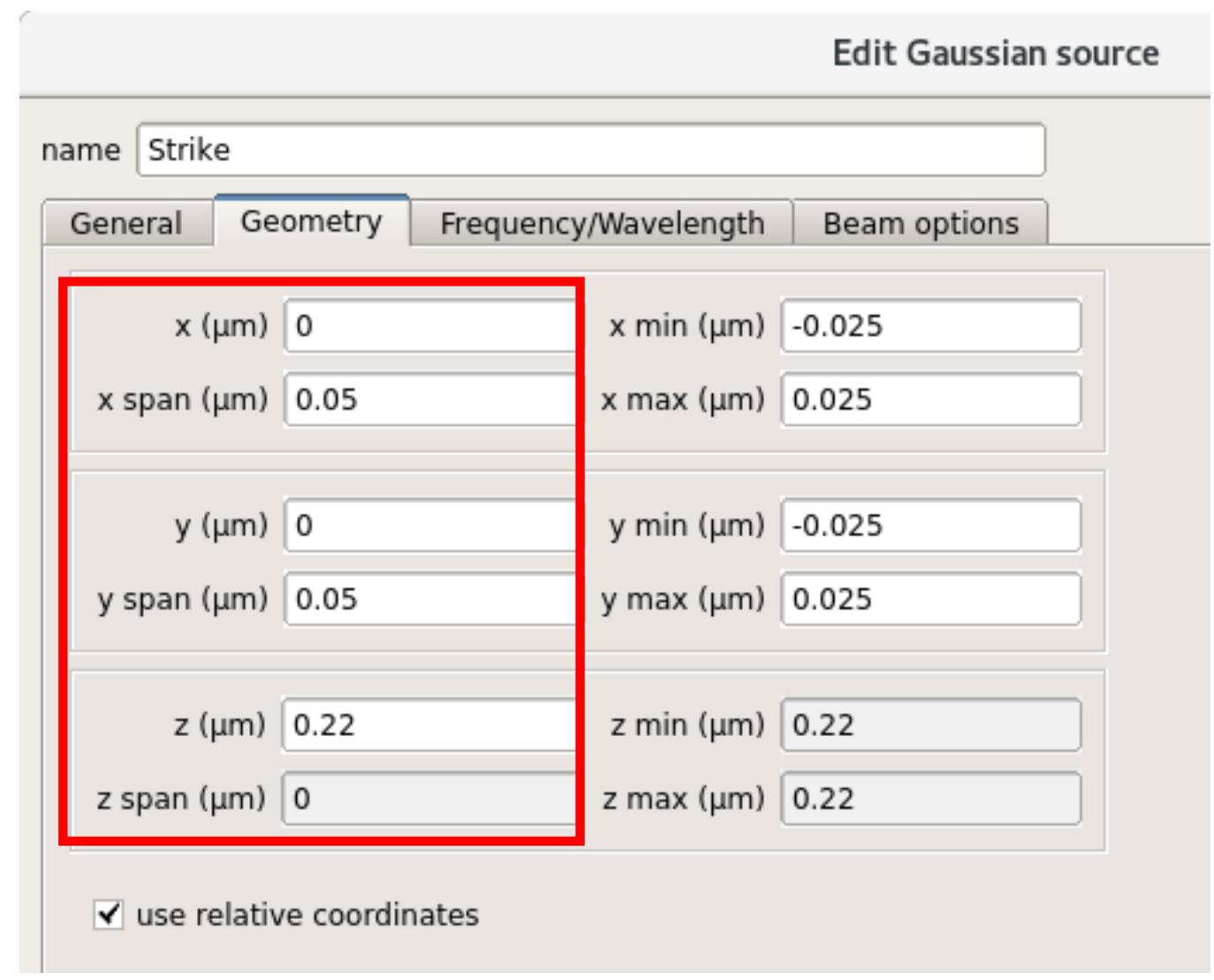

Figure 20

The gaussian source geometry settings. It is set to be a "z-normal" source so that it injects light in the $\mathrm{z}$ direction and is set to be a diameter of about $50 \mathrm{~nm}$ with "x span" and "y span". The $\mathrm{z}$ coordinate is set to $220 \mathrm{~nm}$ to place it on top of the waveguide 


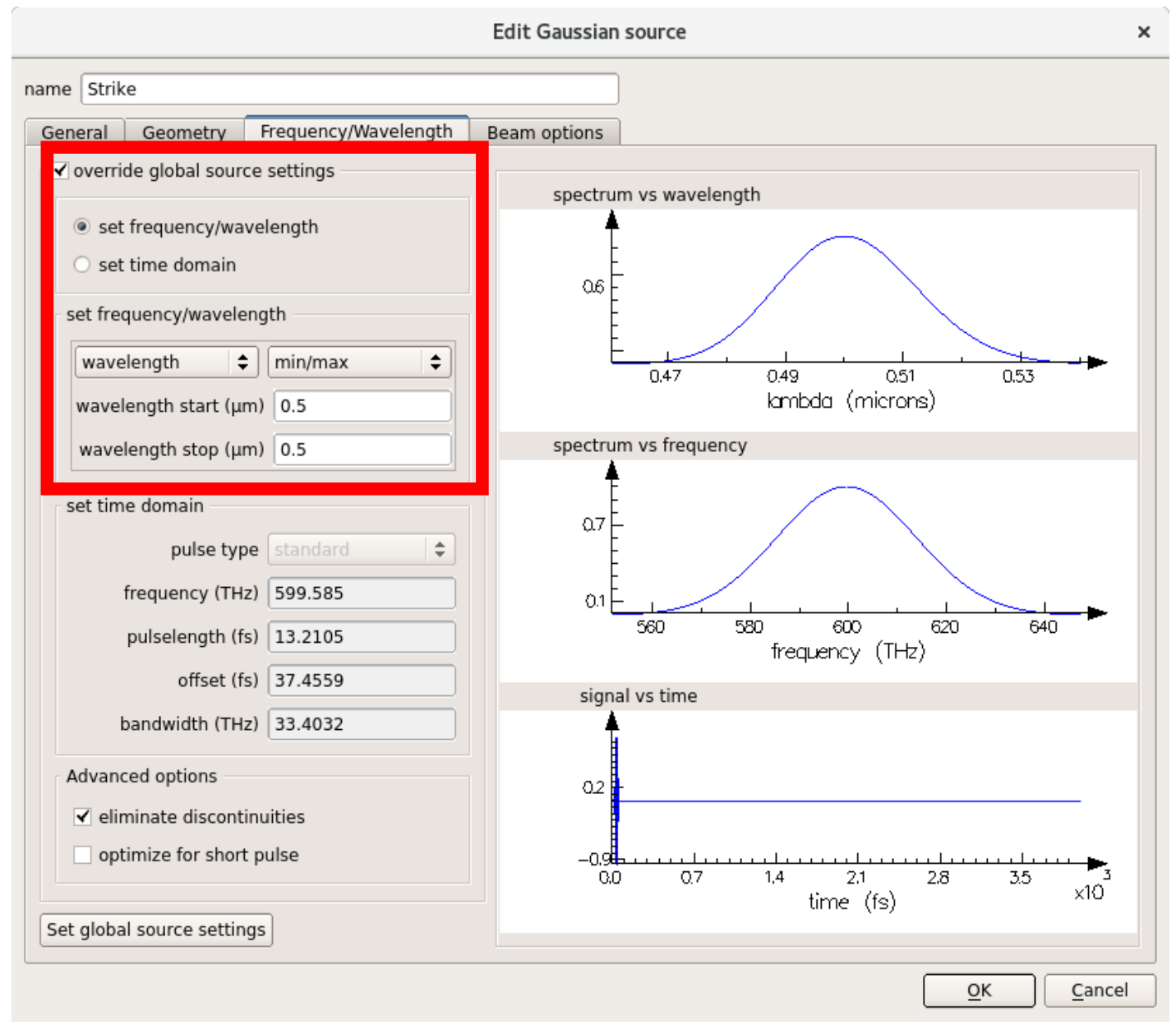

Figure 21

The gaussian light source wavelength settings. "Wavelength start" and "wavelength stop" have been set as to the same wavelength of $500 \mathrm{~nm}$ to achieve as close as possible to a pure $500 \mathrm{~nm}$ light signal

\section{Power Absorption Monitor}

A power absorption monitor object is found in Lumerical FDTD's object library as shown in Figure 22. This object is added to the simulation and is given the same physical dimensions as the simulation region in Figure 15. This object contains a prewritten script that computes the total absorbed power per unit volume profile that is necessary for this simulation methodology. 


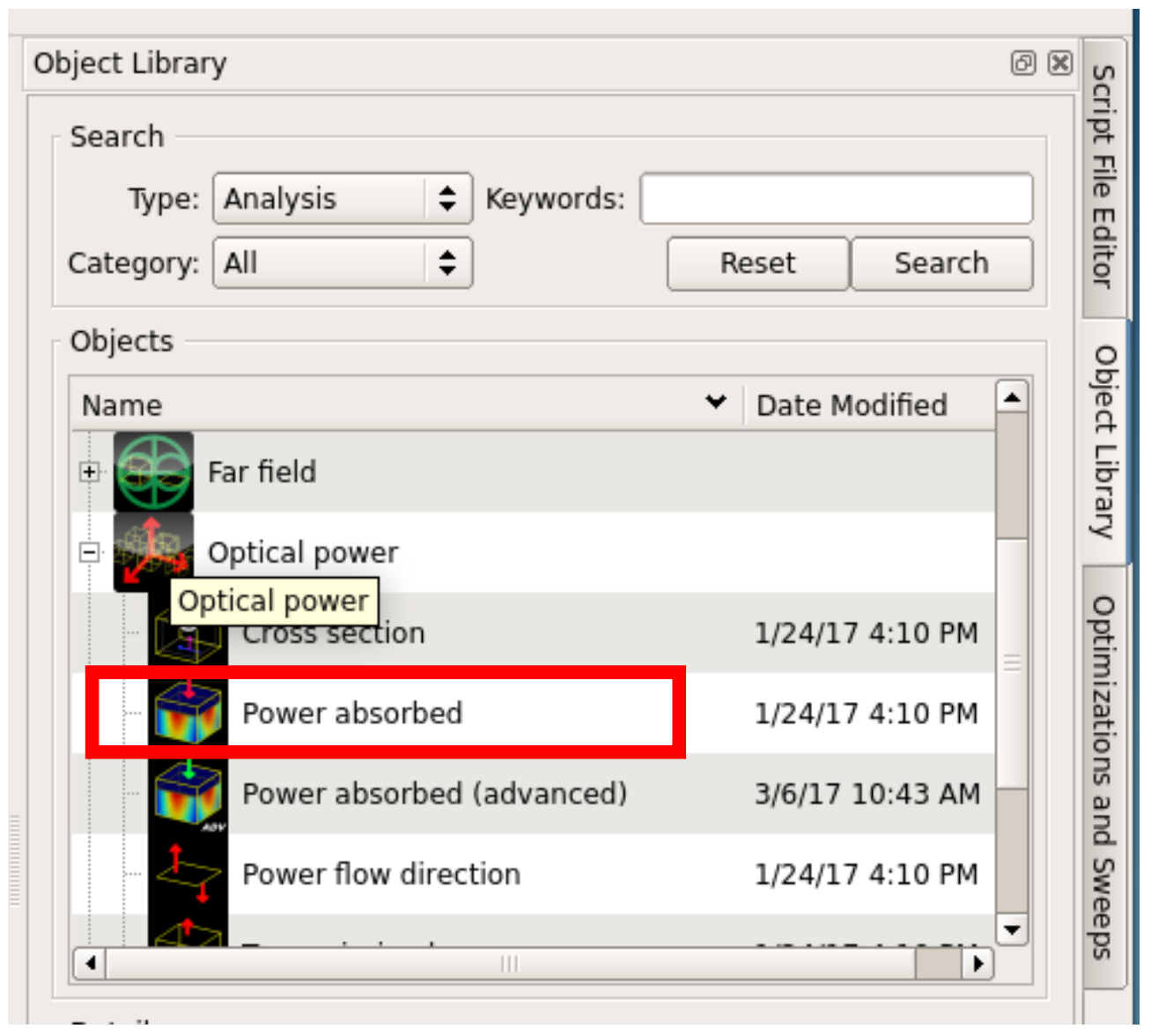

Figure 22

The power absorption monitor can be found in Lumerical FDTD's Object Library under "Optical Power". The non-advanced option is all that is necessary for these simulations

\section{Simulation Execution}

Once all the necessary objects and tools have been added to the simulation region, the simulation may be run. When the simulation is complete, the power absorption profile will be stored in the power absorption monitor and may be saved to a MATLAB (.mat) file. A crosssection of the power absorption profile at the center of the strike $(y=0)$ computed for the waveguide described in Figure 10 with a laser wavelength of $500 \mathrm{~nm}$ representing the ion strike is shown in Figure 23. The power absorption computed here is directly proportional to the generation 
rate of EHPs in the Si waveguide, and as such, the absorption profile may act as a generation source of EHPs in stage B of this methodology.

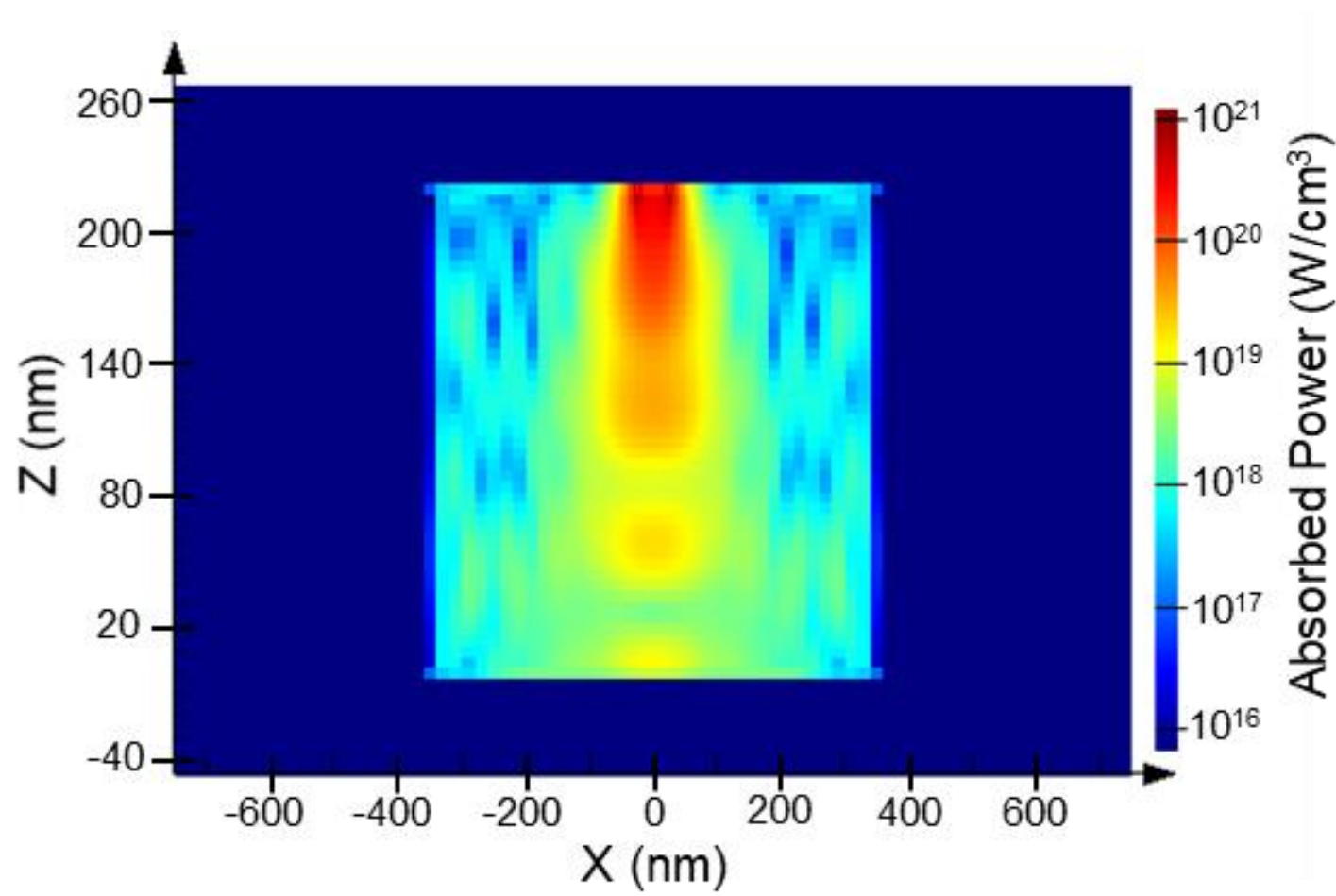

Figure 23

A cross-section of the waveguide showing the optical power absorbed frome the light source at the point where it is at full intensity. This power absorption profile gets converted to an electronhole pair generation rate profile for use in the Lumerical DEVICE simulation to act as an emulation of an ion strike in the electrical domain simulation

\section{Stage B - Charge Transport Simulation}

Following the simulation of the heavy ion strike via analysis of optical absorption, the power absorption profile is imported into Lumerical DEVICE to act as an EHP generation source in the charge transport simulation, step B of Figure 9, and excite a profile of EHPs in the device. DEVICE then simulates the diffusion and recombination behavior of these free charges in the device to capture the electrical response of the ion strike. The output of this stage is a set of 
progressively diffusing discrete-time charge profiles that characterize the charge state of the waveguide over a set of discrete-time points. Once this time-dependent charge profile is computed, it may be imported into stage $\mathrm{C}$ of this methodology to characterize to optical response of the photonic device to an ion strike.

Device Setup

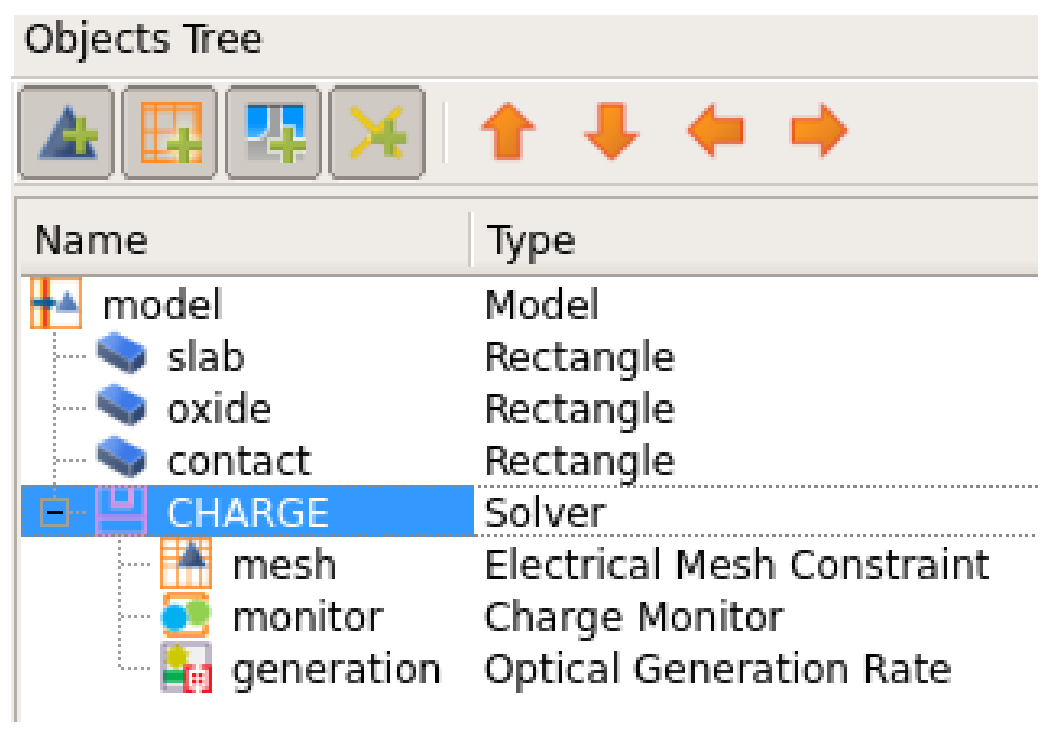

Figure 24

The Objects Tree in Lumerical DEVICE for the charge transport simulation

The device dimensions and material in this simulation are designed identical to the device in the previous simulation (shown in Figure 10) with the exception, in this example, being the waveguide length along the y direction, which is set to about 50 um for this simulation. This allows the generated charges to have some room along the length of the waveguide to diffuse. Otherwise, the diffusing charges would hit the hard simulation boundaries at the end of the waveguide and have nowhere else to diffuse. If the waveguide is not made long enough, the 
charges will eventually hit the two ends of the waveguide and prevent the charges from diffusing any furture due to the lack of boundary conditions in the charge transport simulation. In some experimentation, electrical contact boundary conditions have been added to the ends of the waveguide in an attempt to make the waveguide appear to be theoretically infinitely long to the simulator, similar to the PML boundary condition in the FDTD simulator. This however did not have the desired result and instead interfered with the simulation by giving an outlet for the charge to diffuse and recombine faster than expected in a passive waveguide.

As in the ion strike simulation in FDTD, the material of the waveguide slab is set to Si and the material of the material encompassing the slab is $\mathrm{SiO} 2$ to keep the device consistent with the previous stage.

\section{Charge Solver Setup}

After the device dimensions and materials are specified, a simulation region must be setup. In Figure 24, the solver called "CHARGE" specifies the dimensions of the simulation region as well as the type of simulation to be carried out and other relevant settings. 


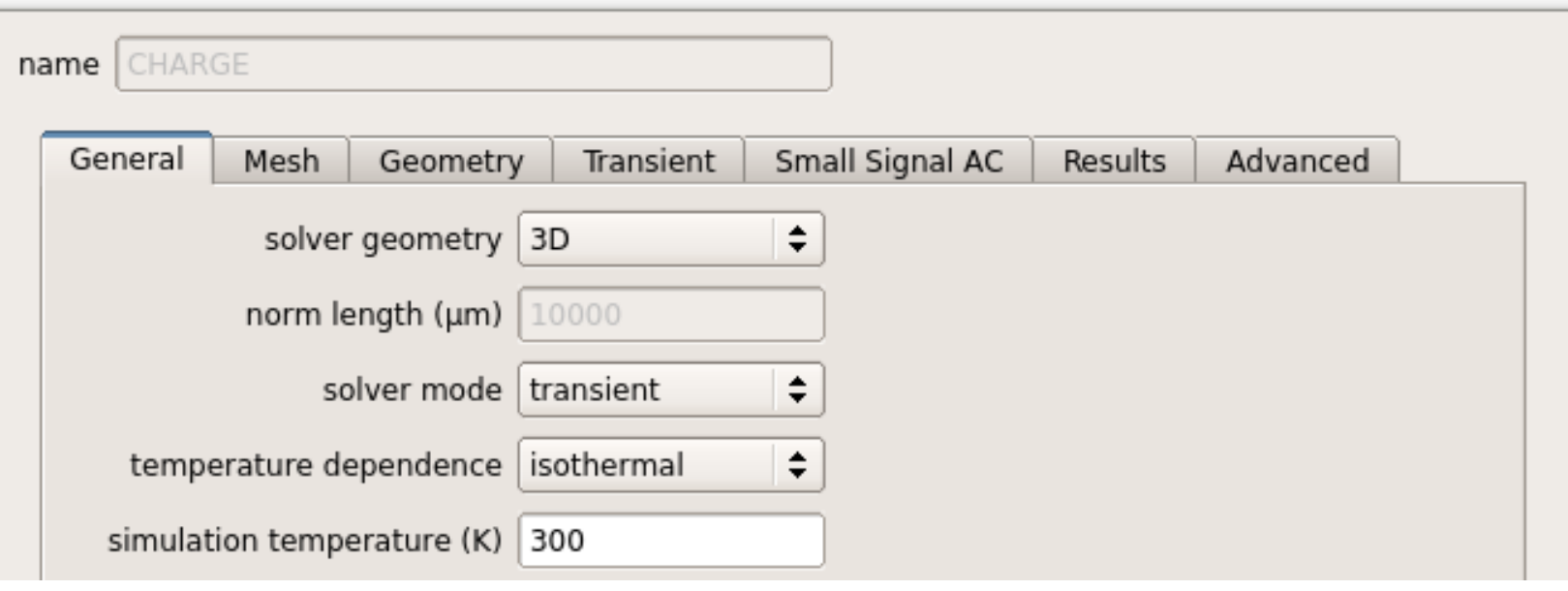

Figure 25

The general charge solver settings. This set to a 3D transient simulation at standard room temperature

Figure 25 shows the generale charge solver settings for this charge transport simulation. This simulation was set to a "solver geometry" three-dimensions. The "solver mode" setting was set to transient to allow this simulation to compute the temporal diffusion of charge in the device. The temperature settings in this thesis are left to their defaults, however, these may be useful in future research where the SEEs in PICs are determined in different temperature environments.

The charge solver's mesh settings are shown in Figure 26. The default mesh edge length has been specified to the solver to be between $0.1 \mu \mathrm{m}$ and $10 \mu \mathrm{m}$. This allows the solver to generate a mesh that contains the mesh edges between those lengths. These values make for a relatively coarse mesh, however a mesh constraint region has been placed directly around the strike location that sets the maximum mesh edge length in a square of $700 \mathrm{~nm}$ around the strike location to $5 \mathrm{~nm}$ (Figure 27). Having this mesh constraint region around the ion strike location is of particular importance in this charge transport simulation, as the charge transport algorithms are particularly 
sensitive to diverging due to fast changing (both spatially and temporally) charge gradients. Such charge gradients are seen in these simulations when generating the impulse of charges that represents the ion strike.

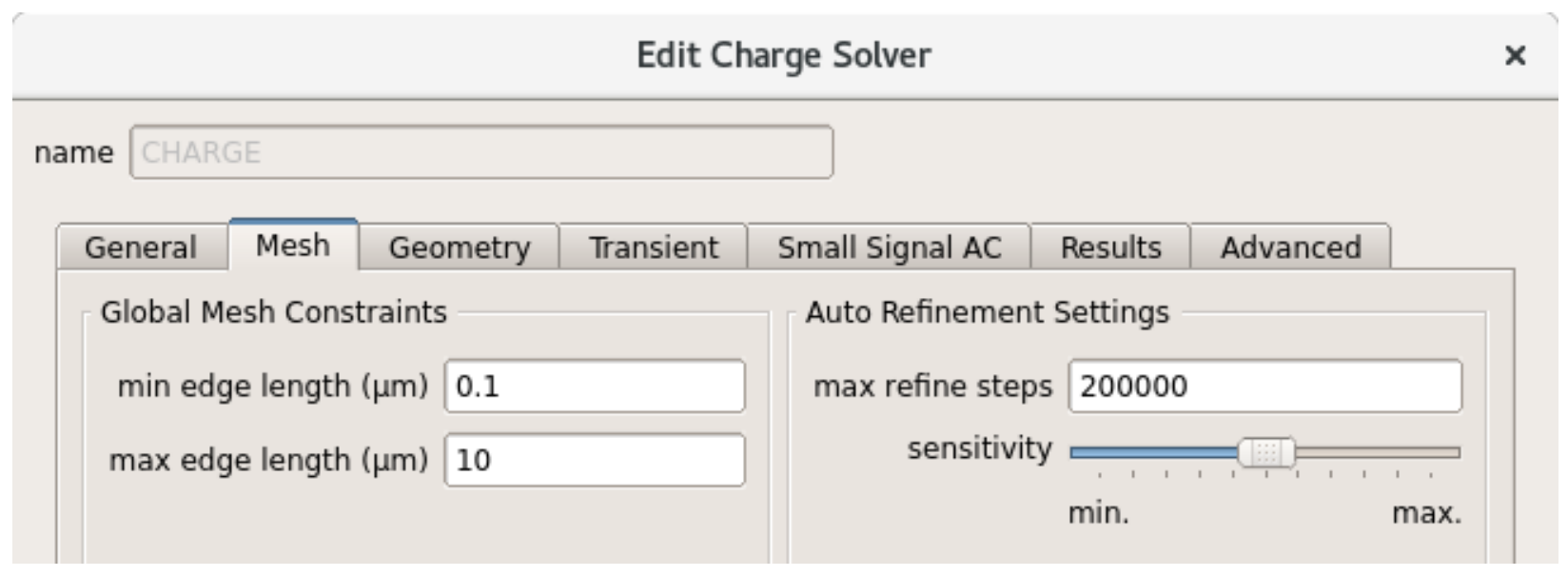

Figure 26

The charge solver mesh settings. The minimum mesh edge length is $0.1 \mu \mathrm{m}$ and the maximum mesh edge length is set to $10 \mu \mathrm{m}$. A long max edge length allows the simulator to generate a mesh that is very coarse in regions far away from the ion strike location to save computational resources. "Max refine steps" has been set to 200000 so that the mesh generator can thoroughly refine the mesh 


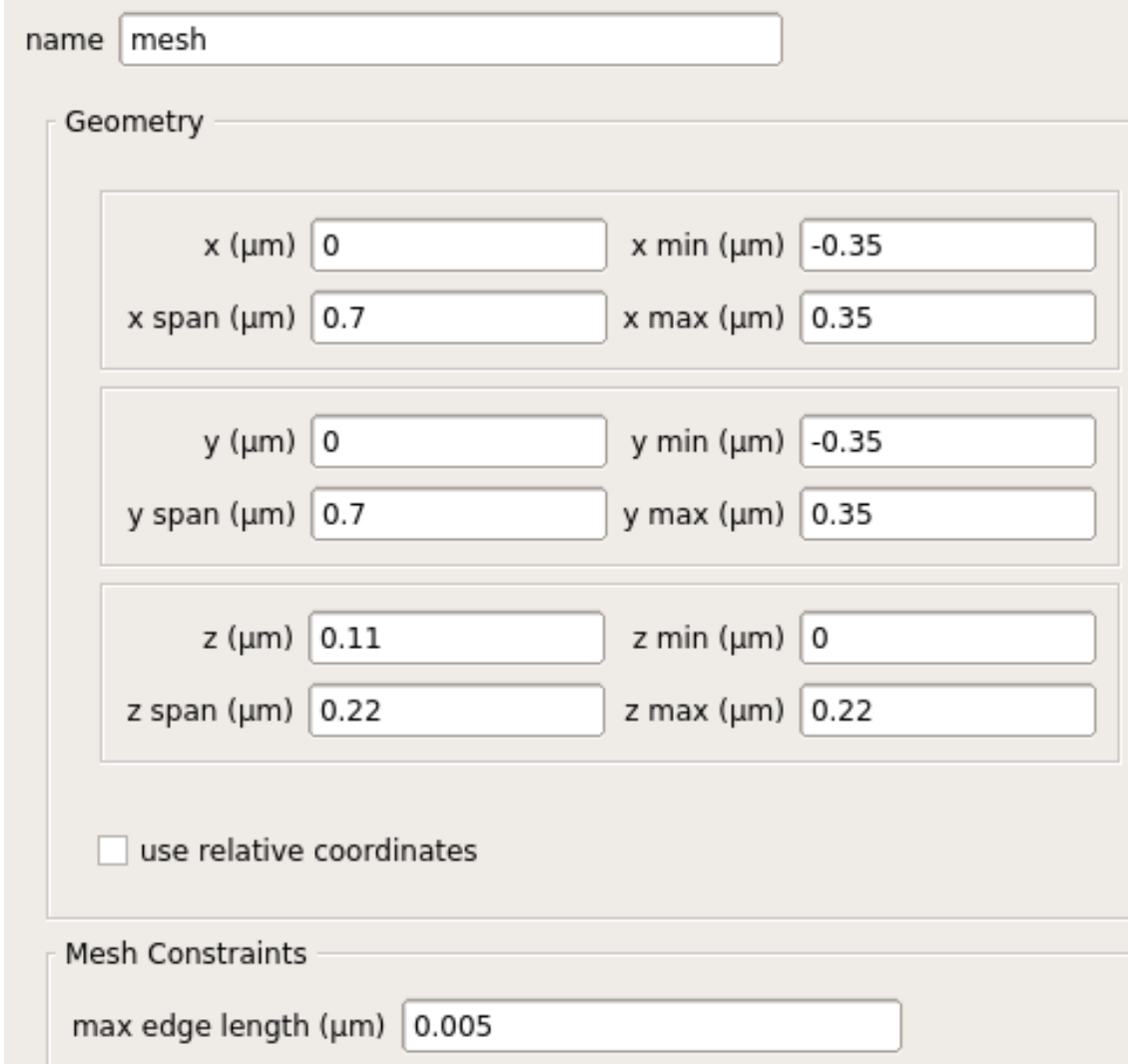

Figure 27

The dimensions and settings for the mesh constraint region directly surrounding the ion strike location. This region prevents simulation divergence by making the mesh finer where the charge gradients are expected to be the steepest 


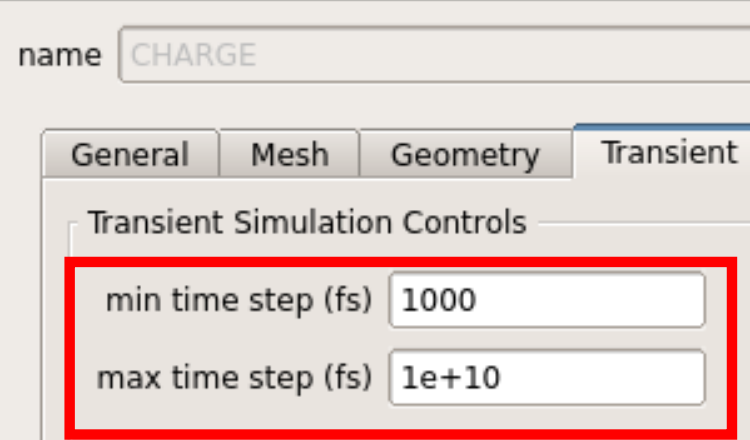

(a)

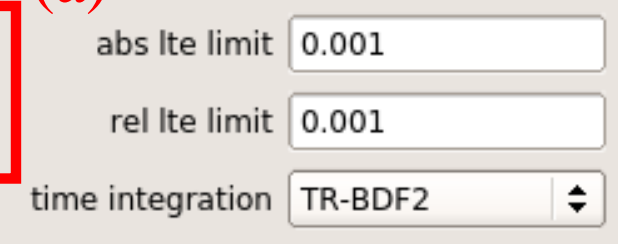$$
\text { rel Ite limit } 0.001
$$

TR-BDF2 \begin{tabular}{|l|l|l|}
\hline Small Signal AC & Results & Advanced \\
\hline
\end{tabular}

\section{Downsampling \\ downsample mode none \\ downsample interval (fs) \\ (b) downsample count \begin{tabular}{|l|l|}
\hline \\
\hline
\end{tabular}}

Global Source Shutter

shutter mode pulse on

shutter ton (fs) 1000

shutter toff (fs) 2000

shutter tslew (fs) 1000

shutter slew function

linear $\div$

shutter slew cutoff

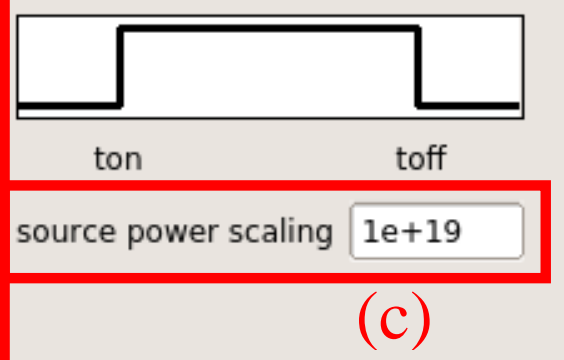

Figure 28

The transient simulation settings for the charge solver. (a) has the minimum time step setting for the simulation set to $1000 \mathrm{fs}$ and the maximum time step set to $10 \mu \mathrm{s}$. (b) is the set of settings that determines the timing of the pulse of charges generated by the charge generation source. (c) scales the intensity of the charge pulse generated such that LET may be tuned in this methodology

Under the "Transient" tab of the CHARGE solver's settings are all of the settings related to how the solver handles the computation of the transient time steps and the generation of EHPs 
by the charge generation sources. Figure 28(a) shows the minimum and maximum time step settings. For this simulation these values have been set to constrain the solver to choose step values between $1 \mathrm{ps}$ and $10 \mu \mathrm{s}$. This allows the minimum step size to be short enough to generate the charges in the device practically instantly (like an ion striking a device) and to capture sufficient detail of the initial charge transient. Setting the maximum time step to $10 \mu$ sives the solver plenty freedom to choose larger time steps as the charge state of the device settles to steady state.

Shown in Figure 28(b) are the settings that determine the timing and shape of the shutter used to control the charge generation source. The shutter mode chosen here is "pulse on" as it allows the user to turn the generation source on for a short time to emulate the instantaneous charge generation of an ion strike. The setting of "shutter ton", the difference between "shutter toff" and "shutter ton, and "shutter tslew" must be greater than or equal to the "min time step" setting in Figure 28(a). Failure to follow this rule will throw an error because the time settings of the global source shutter cannot physically be less than the smallest possible simulation time step. "Shutter tslew" determines the time that it takes for the source shutter to go from zero intensity to maximum intensity. "Shutter ton" determines the time at which the shutter begins to allow charges to generate and "shutter toff" is the time the shutter begins to ramp the charge generation down. "Shutter slew function" determines the shape of the ramp as the shutter turns on and off with choices of exponential and linear. The setting in Figure 28(c) is a global source intensity scaler that multiplies the entire charge generation profile by its value and is useful for LET tuning. These settings as they are shown in Figure 28(b) generate a triangular pulse starting at $1 \mathrm{ps}$ in the simulation, hits peak generation at $2 \mathrm{ps}$, and ramps back down to no charge generation at $3 \mathrm{ps}$. 


\section{Generation Source}

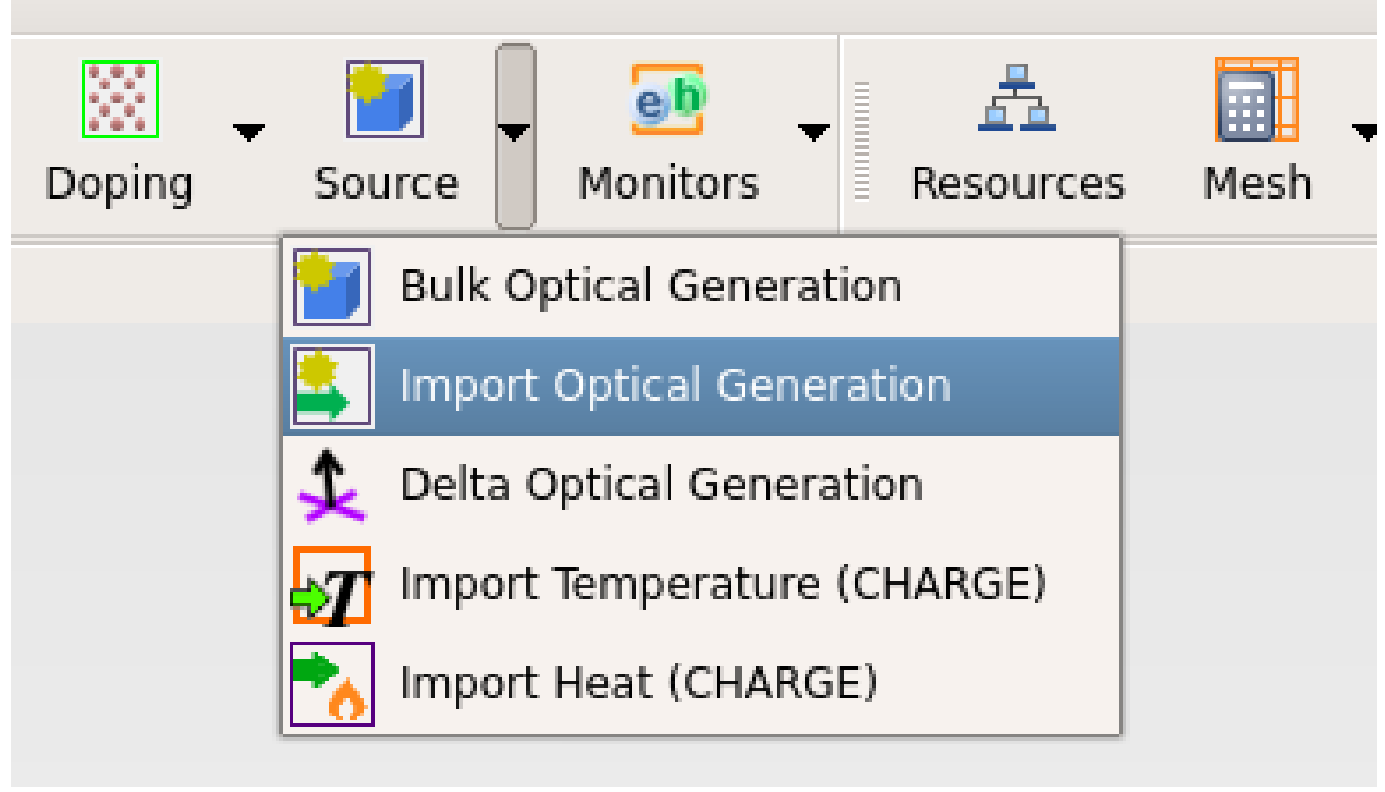

Figure 29

Adding an optical generation source to the simulation region

A generation source may be added to the simulation area by the "Import Optical Generation" tool under "Source" as shown in Figure 29. This is the simulation object allows a power absorption profile from the previous simulation (Figure 23) to be imported into Lumerical DEVICE and used as a source of EHPs for transient simulations. To allow this generation source to be used, the file exported from the power absorption monitor in the previous FDTD ion strike simulation must be imported into the generation source. Once imported, the dimensions of this source are automatically set identically to the dimensions of the power absorption monitor from the previous optical ion strike simulation. If the device dimensions have been set identically to the device in FDTD, then the imported generation profile should line up appropriately with the device specified in DEVICE. 


\section{Si Recombination Settings}

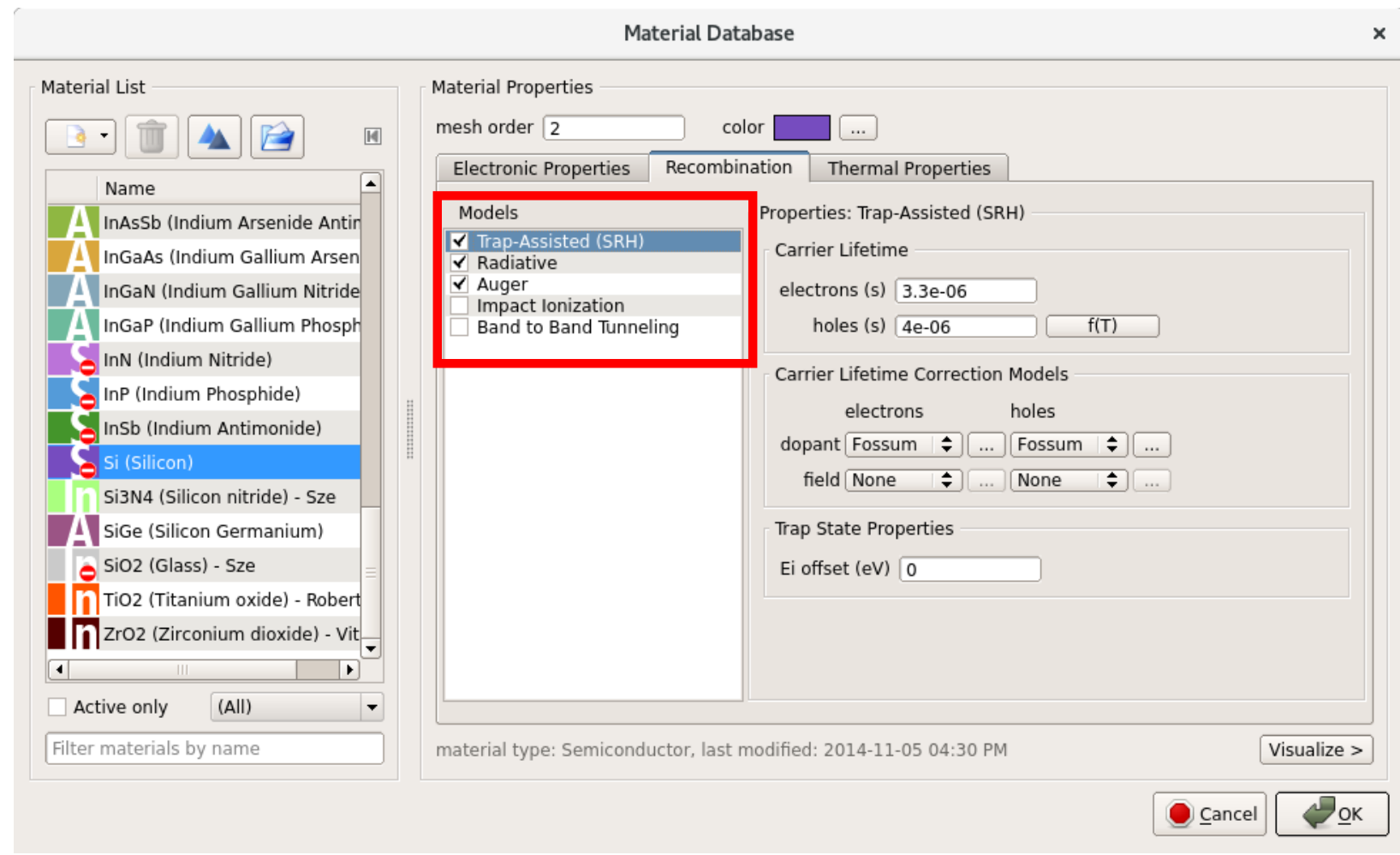

Figure 30

Si recombination settings of the charge solver. The various type of recombination in Si may be enabled and disabled here. The default settings of each type of recombination are already set appropriately for $\mathrm{Si}$

Accurate charge transport simulation in semiconductors requires that recombination be taken into account. The material settings for semiconductors in Lumerical DEVICE have a tab for controlling each of the recombination mechanisms within the semiconductor (Figure 30). The red box in Figure 30 shows where each recombination mechanism may be enabled or disabled. The default settings within each mechanism should already be set to values appropriate to model recombination within $\mathrm{Si}$, it is simply up to the user to determine whether each mechanism is significant enough to enable for each application. If a given mechanism is determined to have a 
negligible effect on the simulation, then it may be disabled here to save computational resources. In this work, the enabled mechanisms have been set to Shockley-Read-Hall (SRH), radiative, and Auger recombination.

\section{Electrical Contact Requirement}

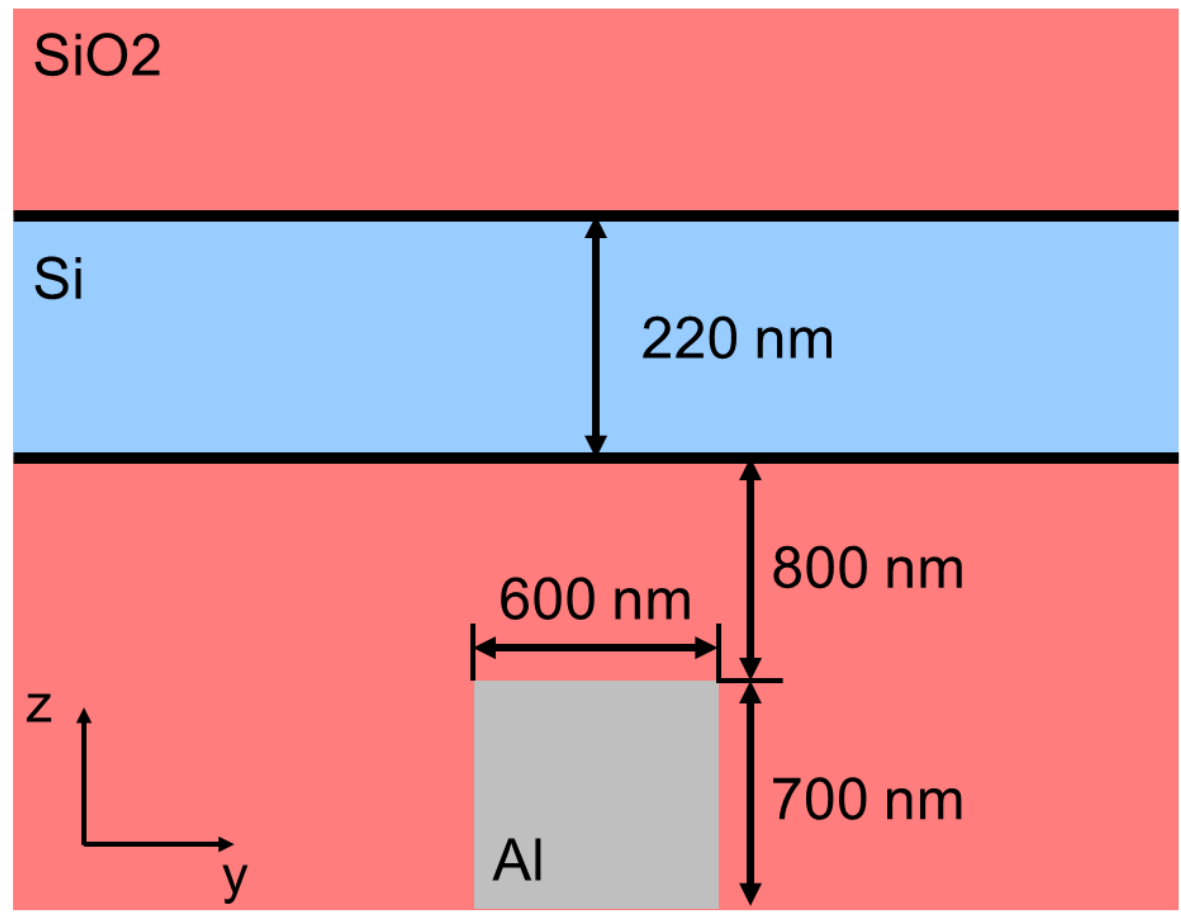

Figure 31

Dimensions and placement of the aluminum contact. It's width in the $\mathrm{x}$ direction is $600 \mathrm{~nm}$ and it is placed directly under the strike location

Lumerical DEVICE requires that at least one electrical contact of a metal material be specified within the simulation region. Since this is an example of a passive optical waveguide, that has no inherent electrical contacts, a dummy contact must be created in a location where it will not have an effect on the natural diffusion of charges. An aluminum contact has been placed 
in the center of the simulation region $(x=y=0)$ with the dimensions as shown in Figure 31 . Notice that the contact has been placed below the waveguide with $800 \mathrm{~nm}$ of $\mathrm{SiO} 2$ between them. This ensures that the aluminum contact is a sufficient distance away from the waveguide as to not affect the diffusion of the charges throughout the waveguide.

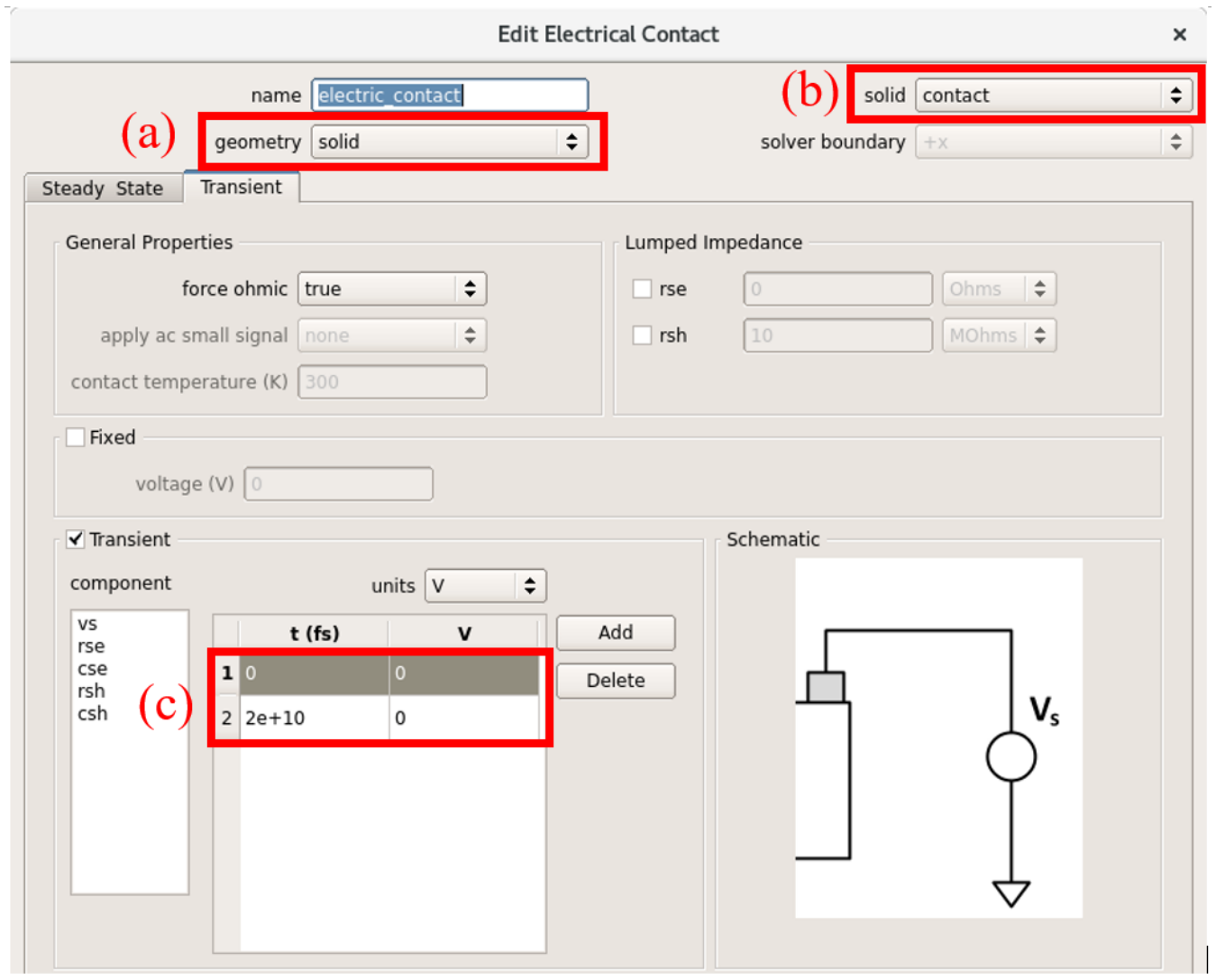

Figure 32

Lumerical DEVICE electrical contact settings. To define a shape in the simulation region as an electrical contact, (a) was set to a solid geometry and (b) was then set to the specific solid name that the user wishes to define as the contact (here it is set to the "contact" as seen in Figure 24). (c1) was set to initial time and voltage of the contact, while (c2) was set to the simulation end time and voltage. Both voltages were set to 0 to ensure that the electrical contact does not affect the passive diffusion simulation in this example 


\section{Charge Monitor}

\section{name monitor}

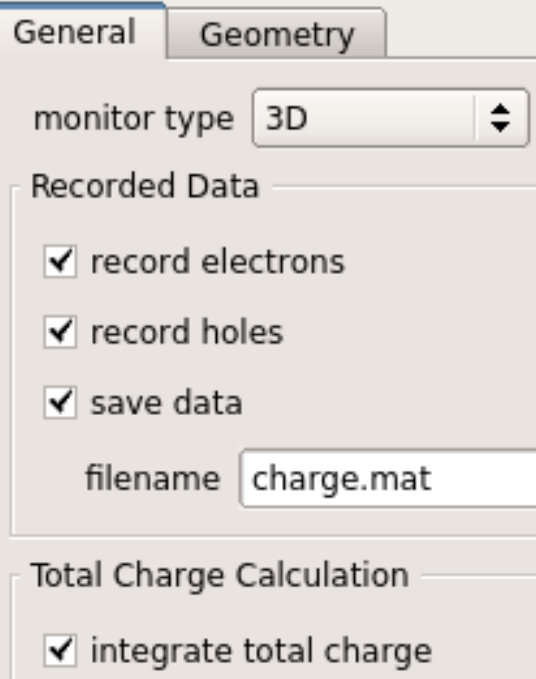

Figure 33

The settings of the charge monitor. "Record electrons", "record holes", "save data", and "integrate total charge" were all checked. A filename was also specified so that the charge profiles recorded by the monitor would be saved

The charge profile information from this simulation may be exported from Lumerical DEVICE by manually exporting it from the CHARGE simulation object once the simulation is complete. However, an easier way to export the charge profiles by using a charge monitor was discovered. In the charge monitor settings, Figure 32, there is a setting to automatically save the contents of the charge monitor to a MATLAB (.mat) file once the simulation is complete. The settings "record electrons" and "record holes" were selected. "Save data" was also selected so that the data was automatically saved from the charge solver into the ".mat" file entered into the "filename" box. "Integrate total charge" was also selected to save a graph of total charge in the 
device overtime for the purpose of estimating the ion strike's LET. The charge monitor has been set to the exact dimensions of the waveguide so that the motion of the charges may be captured in all parts of the waveguide.

\section{Simulation Execution}

Once the simulation has been executed, the charge profile results will be stored in the CHARGE solver and the charge monitor. If the charge monitor was also set to save the results automatically, then the charge profiles will be available in a MATLAB file within the same directory that the simulation file resides. An example of a set of charge profiles, generated in one of these simulations, is shown in Figure 34. The color scale of the charge profiles in Figure 34 is renormalized at each time step to better visualize the diffusion of the charge throughout the device. These charge profiles are used in the following FDTD simulation to compute perturbations in the refractive index of the waveguide and ultimately compute the overall effect on the transmission and phase shift in the waveguide. 


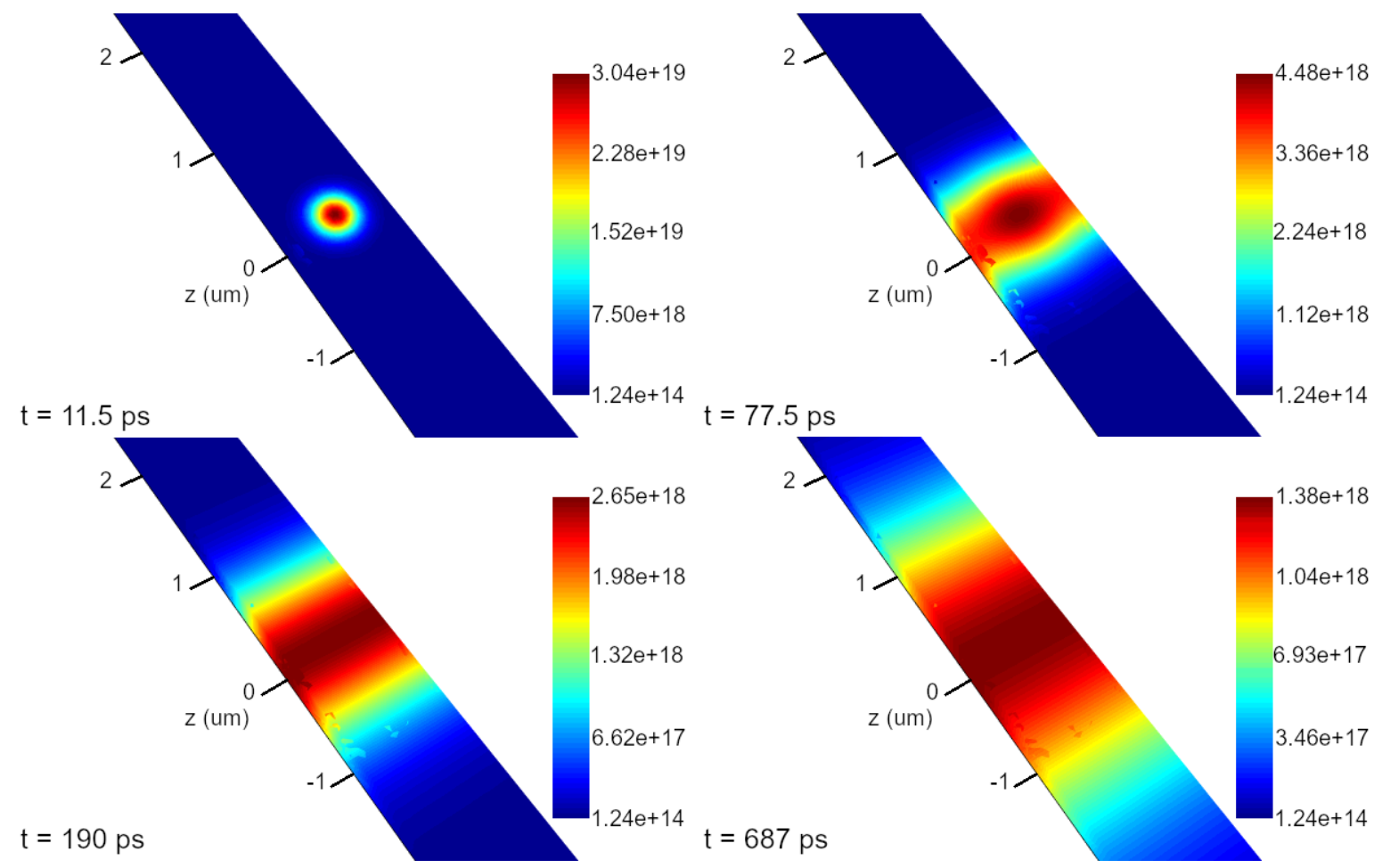

Figure 34

An example set of charge profiles computed by Lumerical DEVICE. As time progresses, the charge spreads out through the waveguide and the peak charge density decreases. These discrete-time charge profile steps are used in the following simulation to compute the transmissive parameters of the waveguide for each time step. Note that the color scale is renormalizing for each time step in this image. This helps to better visualize the diffusive nature of the charge

\section{Estimating and Tuning LET}

The LET of the ion strike was estimated via analysis of the charge profiles, and tuned by adjusting the generation source intensity setting shown in Figure 28c. One method of estimating the LET is to enable the "integrate total charge" setting in Figure 35. This setting stores an array of results that can be displayed as total electrons and total holes in the waveguide over time. The LET was computed via Equation 3.1 by assigning the value of $N_{t}$ as the maximum amount of the electrons. The length $l$ in Equation 3.1 is set to $220 \mathrm{~nm}$ in this case. $l$ represents the path length 
of the ion in path's length in the device, which may change depending on the ion strike angle and strike location.

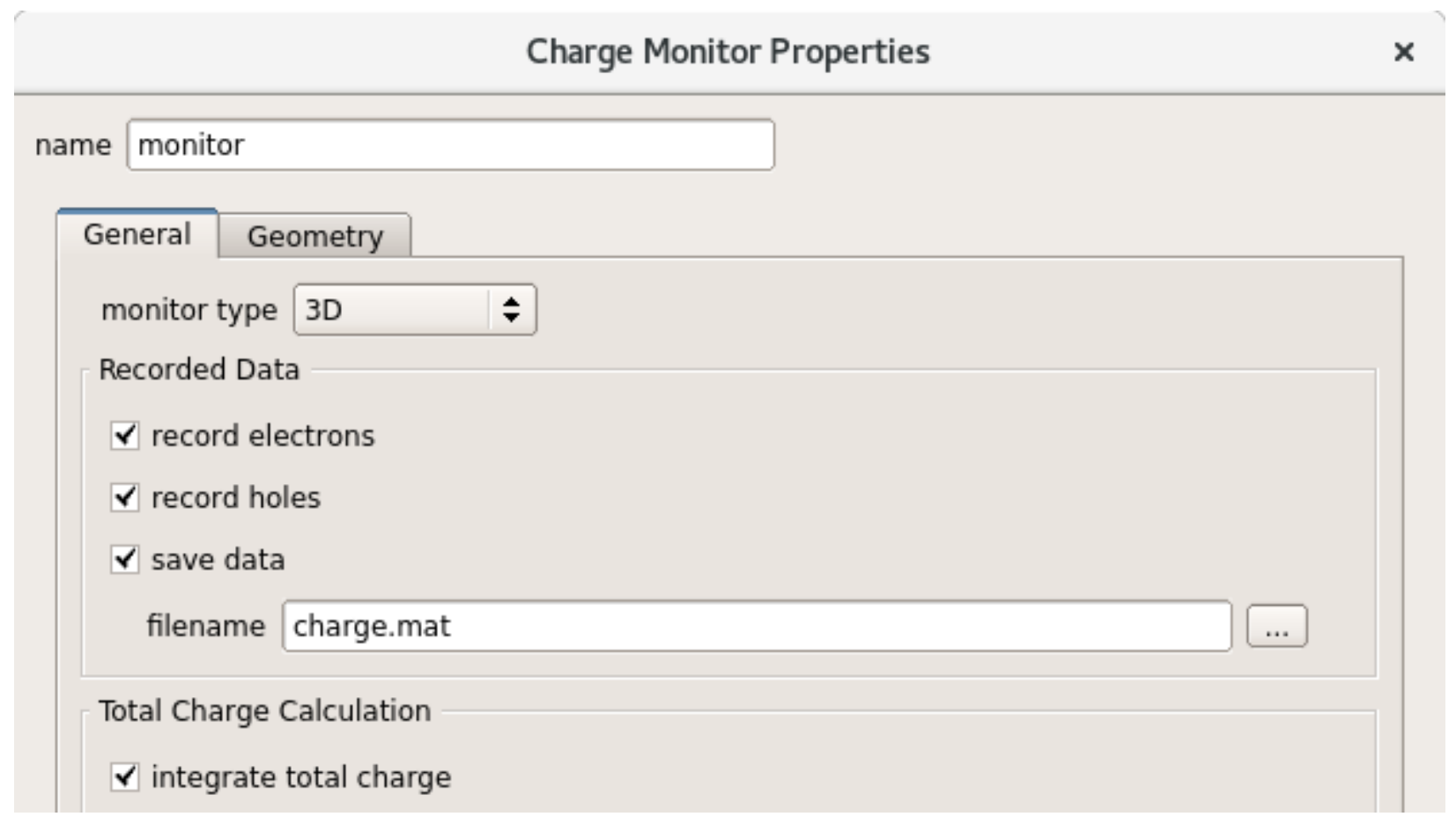

Figure 35

The settings of the charge monitor. Both "record electrons" and "record holes" must be selected. "Save data" allows the charge monitor to automatically save the computed charge profile to a file named in the "filename" field. "Integrate total charge" computes the total number of electrons and holes within the charge monitor which is convenient for estimating LET

One problem encountered with the "integrate total charge" function in Lumerical DEVICE's charge monitors, is that it is only accessible to charge profiles that were just computed. If the Lumerical DEVICE software is closed or a new simulation is started, then a given charge profile can no longer be put through DEVICE's charge integrator to compute the total charge. If estimating the LET was forgotten before closing the software, then it could not be done for the charge profile that was just computed. An external charge integration script was developed in 
MATLAB to fix this problem and is shown in Appendix A.1. The DEVICE generated output charge profile may be imported into this script to compute the total charge separately from the Lumerical software. This script has the added benefit that it not only computes the total charge but includes a line of code to automatically estimate the LET from the given charge profile.

\section{Mesh Analysis}

A mesh analysis was performed to ascertain the effect of the mesh size on the results of the DEVICE simulation. This was done by running a set of example simulations that kept all parameters constant except for maximum mesh length of the mesh constraint region in Figure 27. This parameter sweep reran the simulation for maximum mesh lengths of $2.5 \mathrm{~nm}, 5 \mathrm{~nm}, 10 \mathrm{~nm}, 20$ $\mathrm{nm}$, and $40 \mathrm{~nm}$. The LET of each resulting charge profile was estimated with the MATLAB script in Appendix A.1 and plotted in Figure 36.

The graph in Figure 36 appears to converge to an LET of about $97.7 \mathrm{MeV}-\mathrm{cm}^{2} / \mathrm{mg}$ as the maximum mesh length gets smaller. This convergence indicates that the simulation is getting more accurate by decreasing the mesh size surrounding the ion strike location. As mesh size increases, the LET becomes increasingly over-estimated. With a $5 \mathrm{~nm}$ maximum mesh length, the estimated LET is very close to converging and the mesh size is not so small cost a lot in processing power. The maximum mesh length of $5 \mathrm{~nm}$ has therefore been deemed sufficient to generate an accurate mesh for these simulations. 


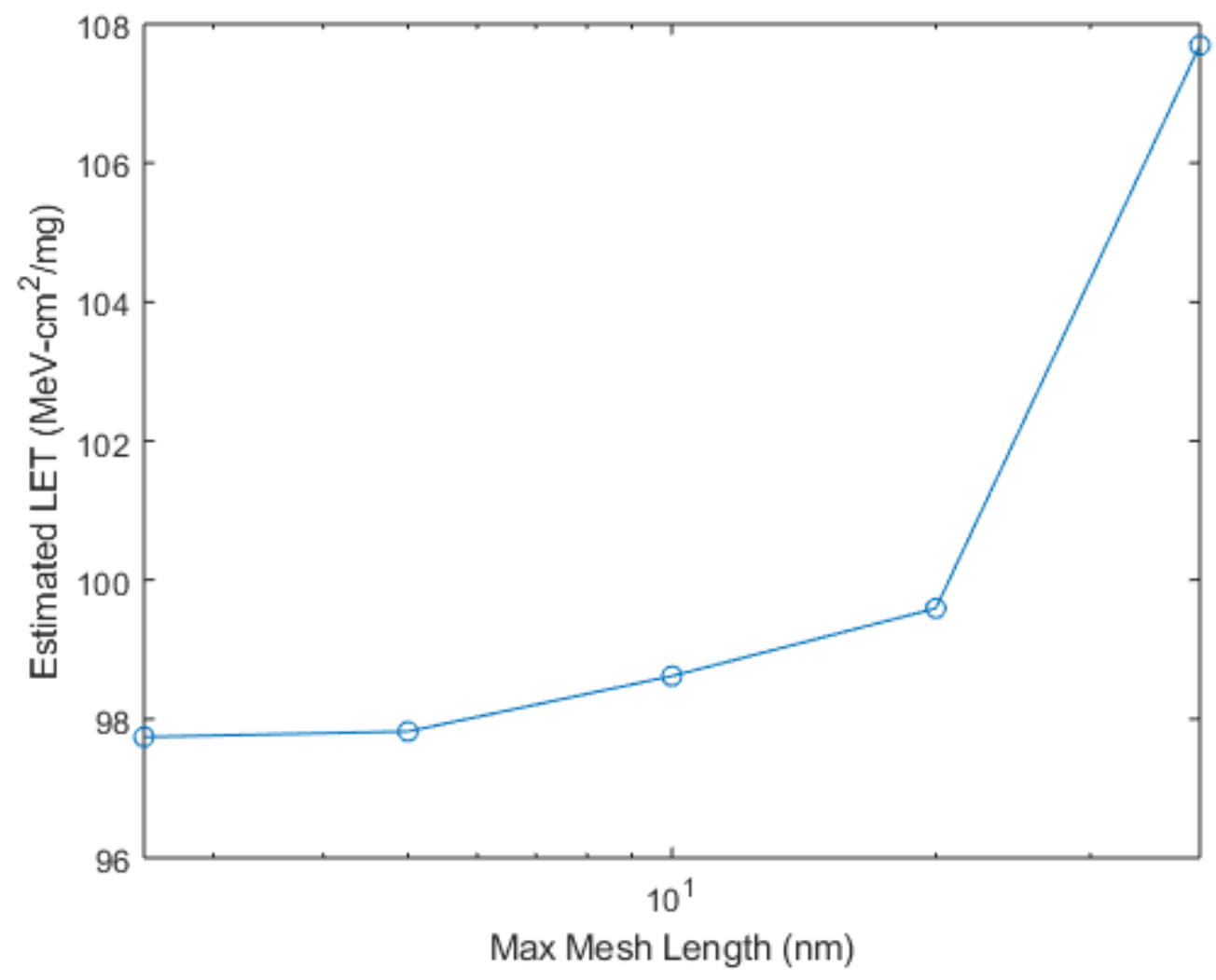

Figure 36

Estimated LET versus maximum mesh length for an example charge transport simulation. The estimated LET converges to an LET of about $97.7 \mathrm{MeV}-\mathrm{cm}^{2} / \mathrm{mg}$ as maximum mesh length is reduced, indicating that the simulation becomes more accurate with reduced mesh size. LET seems to be over-estimated at max mesh lengths larger than $5 \mathrm{~nm}$

\section{Stage C - Extraction of Losses, Phase Shift, and Optical S-Parameters}

The final stage of this metholodgy starts by importing the the dynamic charge profile generated in the previous stage into FDTD, and using it as a means of computing refractive index shifts within the device caused by the free carriers. The Soref \& Bennett charge-to-index conversion model is implemented in FDTD to handle this conversion. Input and output ports are setup within the device and used as a means of extracting the s-parameters of the device that contain information of forward transmission losses as well as phase shifts. A simulation sweep is 
setup to sweep through the time index of the charge profile and gather the s-parameters from the input and output ports as the time index progresses. This generates an array of s-parameters over time that may be converted transmission loss and phase shift versus time, and thus have a timedomain characterization of a SEE in the photonic device.

Device Setup

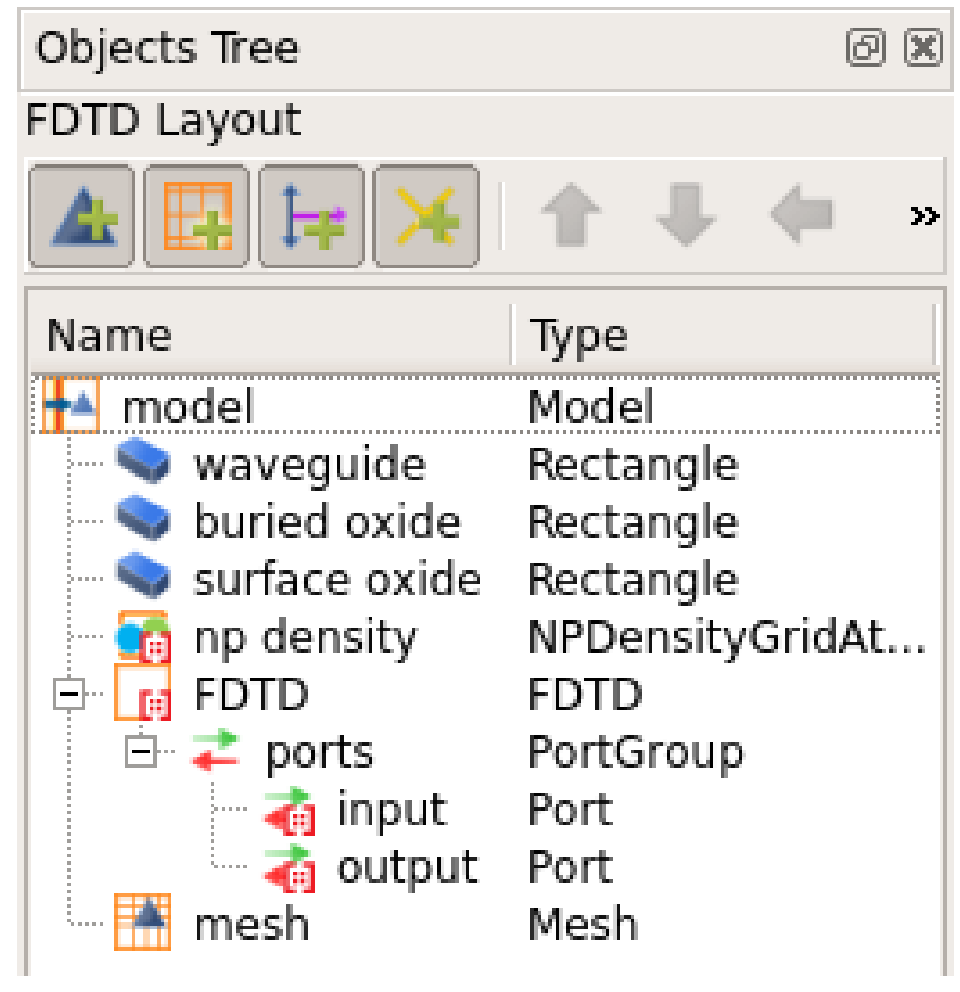

Figure 37

The Objects Tree in Lumerical FDTD for the s-parameter extraction simulations. The waveguide and mesh is setup identically to the device in Figure 14. There is now an "np density" object for including the carrier profiles in FDTD. The FDTD simulation object now includes input and output ports that facilitate the extraction of device's s-parameters 


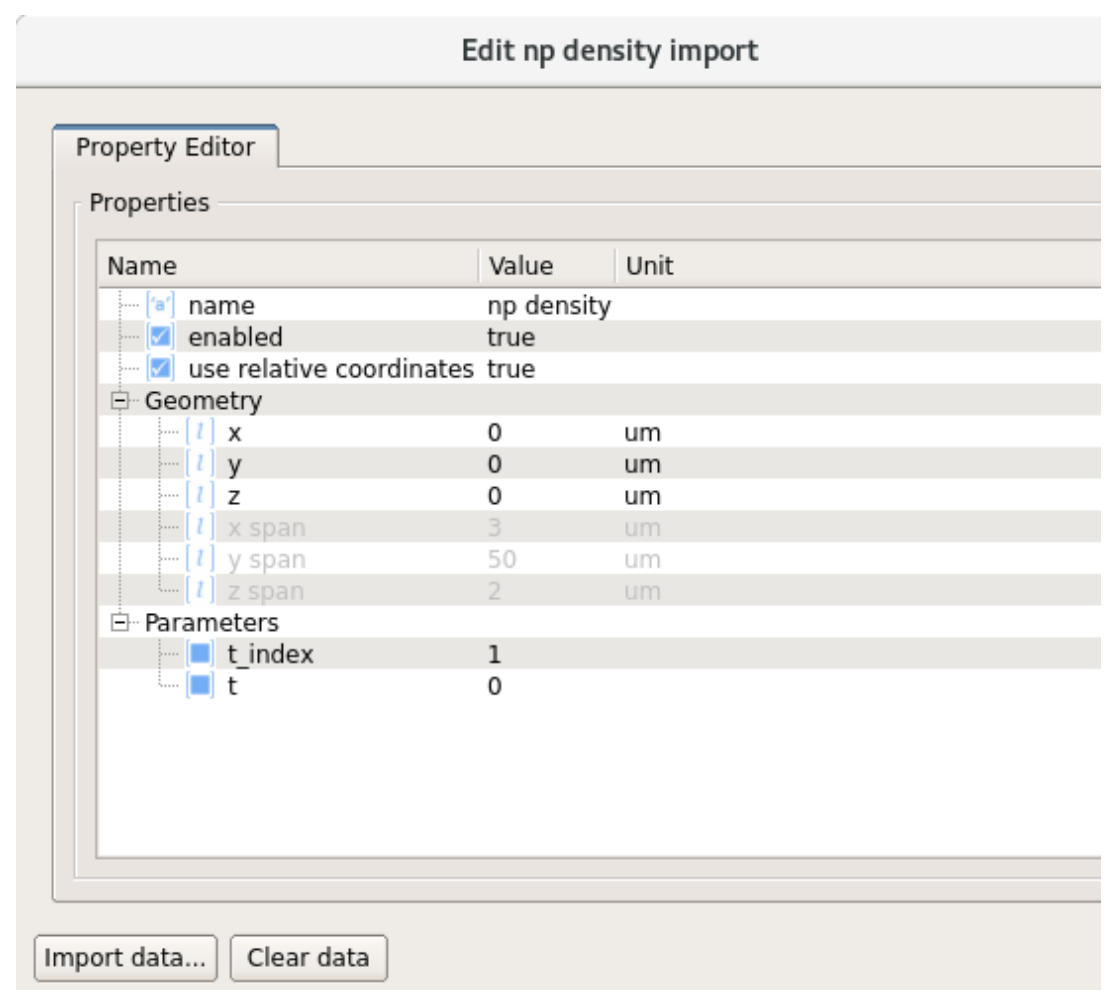

Figure 38

The properties of the "np density" object. This object allows the user to import a set of charge profiles generated by Lumerical DEVICE through the "Import data..." button

The objects tree from this simulation stage, and the simulation objects necessary for extracting the s-parameters are shown in Figure 37. The first object of interest that is unique to this stage is the "np density" object which provides a means of importing the charge profiles generated in the previous simulation stage into FDTD. The np density object supports timevarying charge profiles by including the "t_index" setting seen in Figure 38 . By varying this index value, the user can effectively choose which time point in the electrical domain is being represented as refractive index shifts in the optical domain. By stepping through select values of this index and extracting the device s-parameters, the time-domain response of the device to the single event may be constructed. 


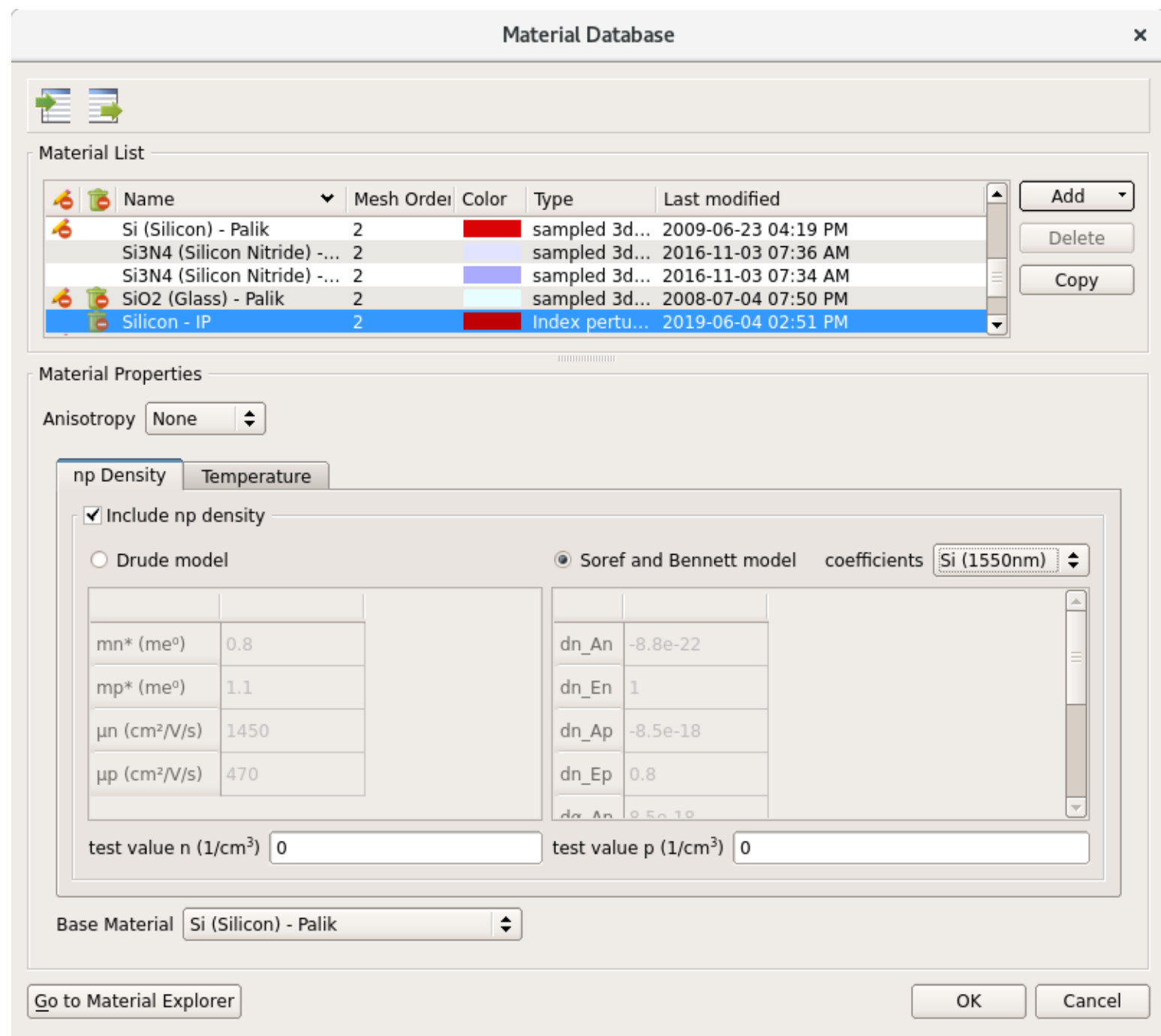

Figure 39

The Si material settings for modeling the shifts in optical properties. A new Si material has been created "Si - IP" and has been set to a material type of "index perturbation" and set to inherit the optical properties of the "Si (Si) - Palik" material. To enable Si's refractive index dependency on free carriers, "include np density" is checked and the chosen model is the "Soref and Bennett model" with $1550 \mathrm{~nm}$ coefficients

The device dimensions here are setup identical to the dimensions of the device in the first stage to keep the simulations consistent. An exception to this is the waveguide length (y-direction) which is set to $\sim 51 \mu \mathrm{m}$ as it is set to in the second stage. Although the dimensions remain mostly the same, the definition of the Si material must be changed to take the refractive index of Si's 
dependence on the free carriers in the np density object into account. A new material must be defined in FDTD's material database (Figure 39) that inherits the base characteristics of the Si material but computes the refractive index perturbations based upon free carriers. The material type "index perturbation" has this functionality and includes a model for the Soref and Bennett charge-to-index conversion model.

\section{Simulation Region Setup}

The simulation region dimensions and settings (Figure 40) are set identical to the first stage's simulation region (Figure 15, Figure 16, and Figure 17) with the exception of the simulation region length which is now $51 \mu \mathrm{m}$. This allows the simulation region to contain the entirety of the $\mathrm{np}$ density object so that it can capture the full set of transient charge characteristics as refractive index shifts. 


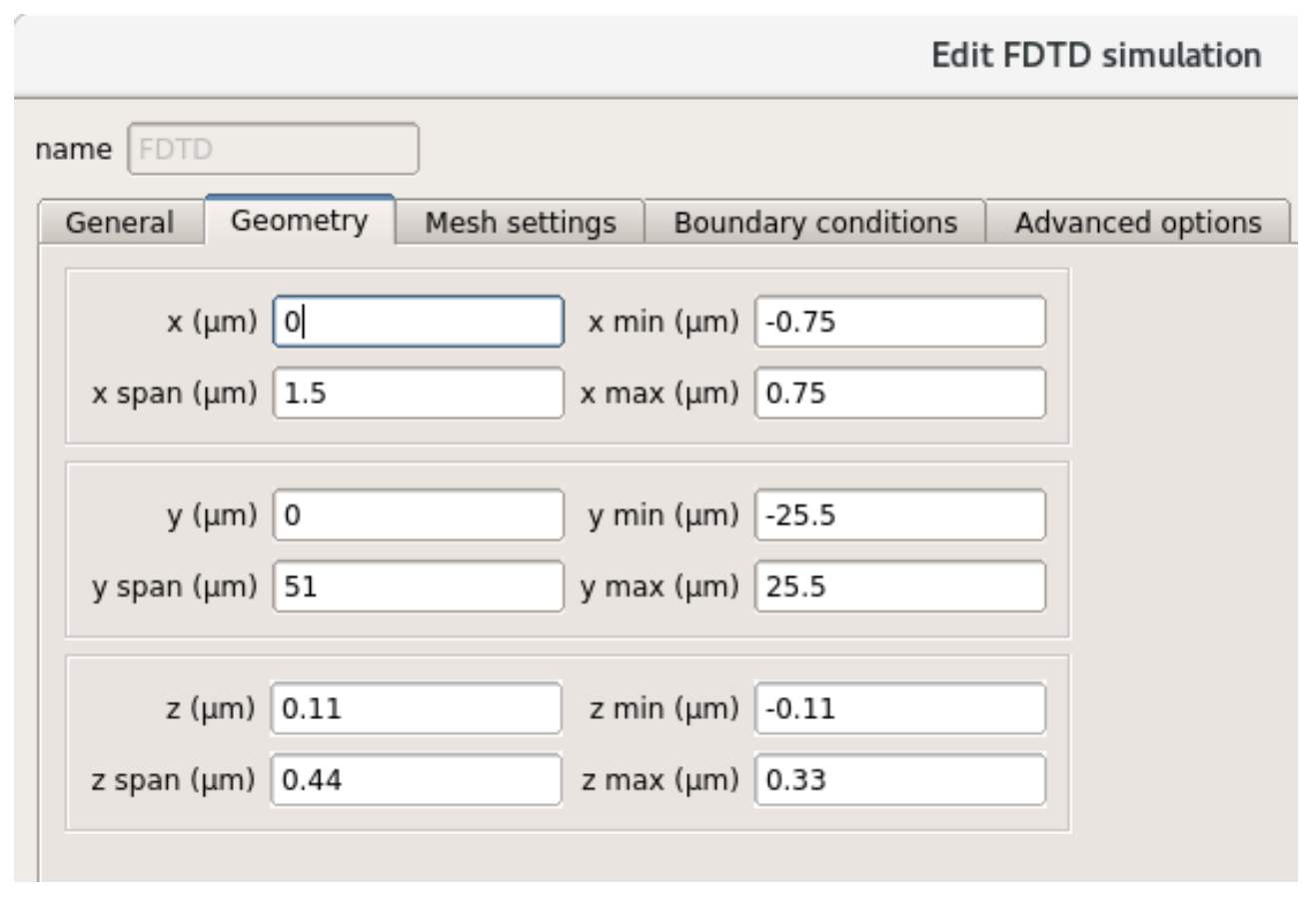

Figure 40

The FDTD simulation region dimensions for the parameter extraction simulation. The length of this simulation region (y-direction) is set to $51 \mu \mathrm{m}$ so that it is slightly longer than the imported charge profile. The other dimensions are identical to the simulation region in Figure 15 making it a $1.5 \mu \mathrm{m} \times 51 \mu \mathrm{m} \times 0.44 \mu \mathrm{m}$ simulation region

\section{S-Parameter Extraction Setup}

The device's s-parameters cannot be extracted without some form of monitors measuring the device input and output. In Lumerical FDTD, these monitors come in the form of ports. This simulation uses two ports (one input and one output), the dimensions of which are shown in Figure 41. These ports have been made larger than the waveguide cross-section so that they sufficiently span the waveguide's modal profile. The input port is placed at one end of the np density profile $(y=-25 \mu \mathrm{m})$ and the output port is place at the opposing end $(\mathrm{y}=25 \mu \mathrm{m})$ to allow the light injected by the input port to come into contact with all induced charges before reaching the output. 


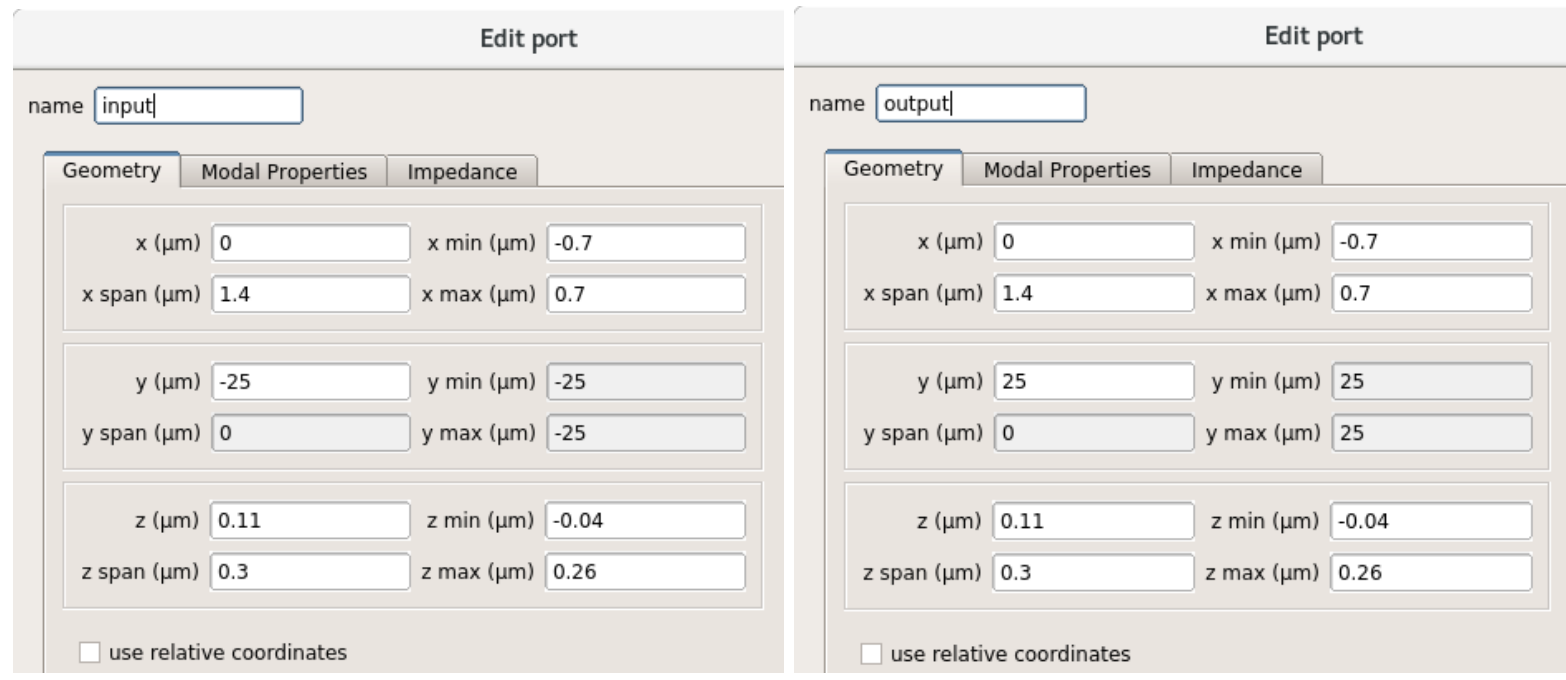

Figure 41

S-Parameter extraction input and output port dimensions. These ports are placed at opposing ends of the waveguide and are made slightly smaller than the overall simulation region but still larger than the cross section of the waveguide so that the ports can generate the whole waveguide mode

Figure 42 shows the modal properties of the input port that determine the mode injected by the port as well as the direction, amplitude, and phase of the signal. So that the light is injected down the length of the waveguide, the "injection axis" is set to "y-axis". Having the "direction" set to "forward" causes the light to be injected in the positive y direction. The default amplitude of $1 \mathrm{~V} / \mathrm{m}$ and phase of 0 are fine as this allows the port to output a normalized wave. The port is set to operate under the waveguide's fundamental mode (TE) over one frequency point that represents a $1550 \mathrm{~nm}$ wavelength (the waveguide's operating wavelength). The output ports modal properties are identical except that its direction is set to "backward". Setting all ports such that they are facing "into" the device is important for keeping the sign of the s-parameters consistent. 


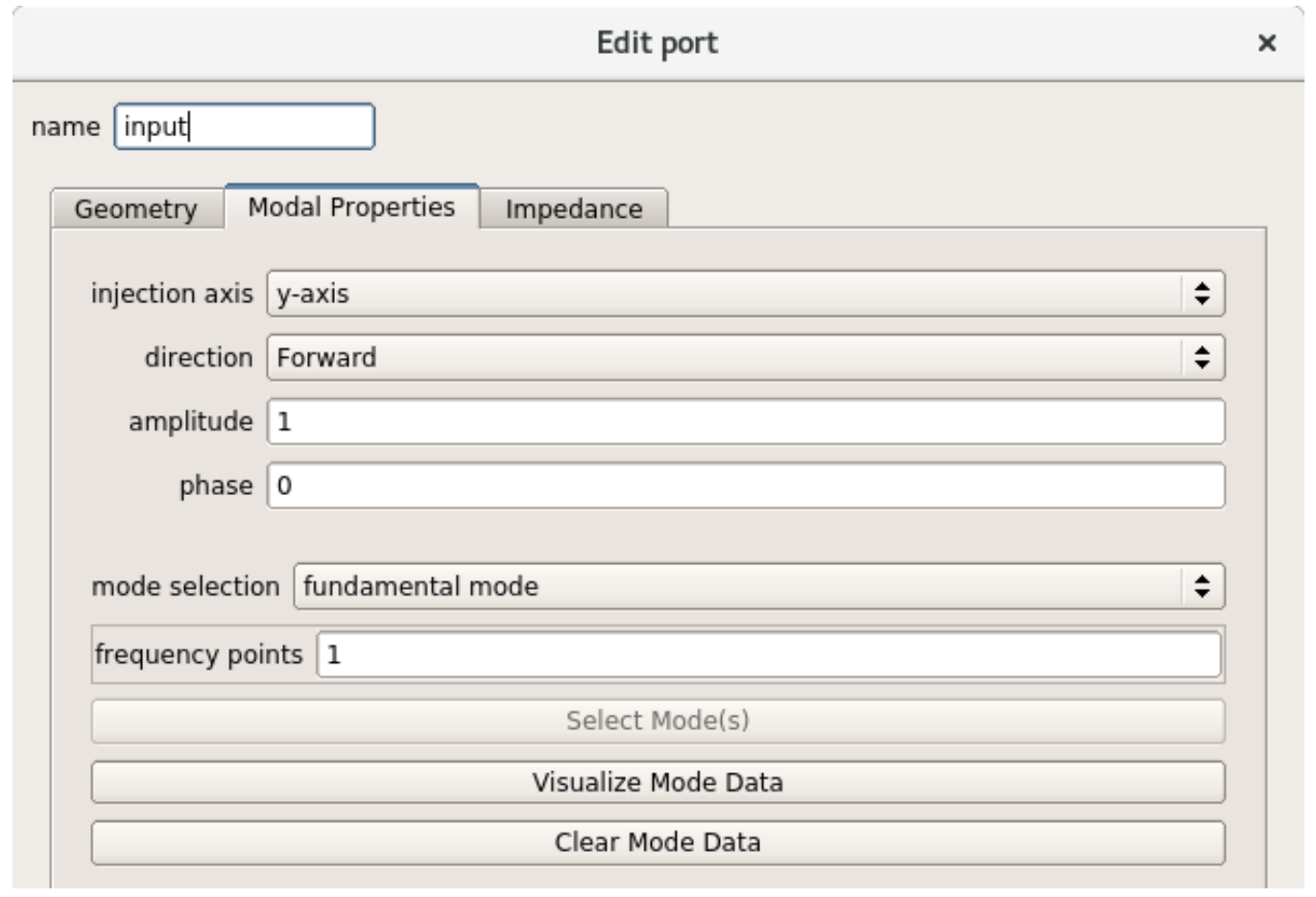

Figure 42

The modal properties of the input port. This port is set to inject forward along the y-axis so that it injects in the positive y direction. The amplitude and phase are set to 1 and 0 respectively to simply act as a normalized electromagnetic wave. The source is operating in the waveguide's fundamental mode over 1 frequency point $(1550 \mathrm{~nm})$

\section{Charge Profile Time Sweep Setup}

All of the tools necessary for extracting a single set of s-parameters from the device have been set up so far. However, the goal of this methodology is to generate a series of s-parameters that represent the changing state of a device over time in response to a single event strike. It is necessary to set up an FDTD parameter sweep to accomplish this. The parameter to be swept is the time index ( $\mathrm{t}$ index) in Figure 38. This simulates the passage of time in the electrical domain that manifests in the optical domain as time-dependent shifts in Si's refractive index, and as a transient in the transmissive properties of the device. 


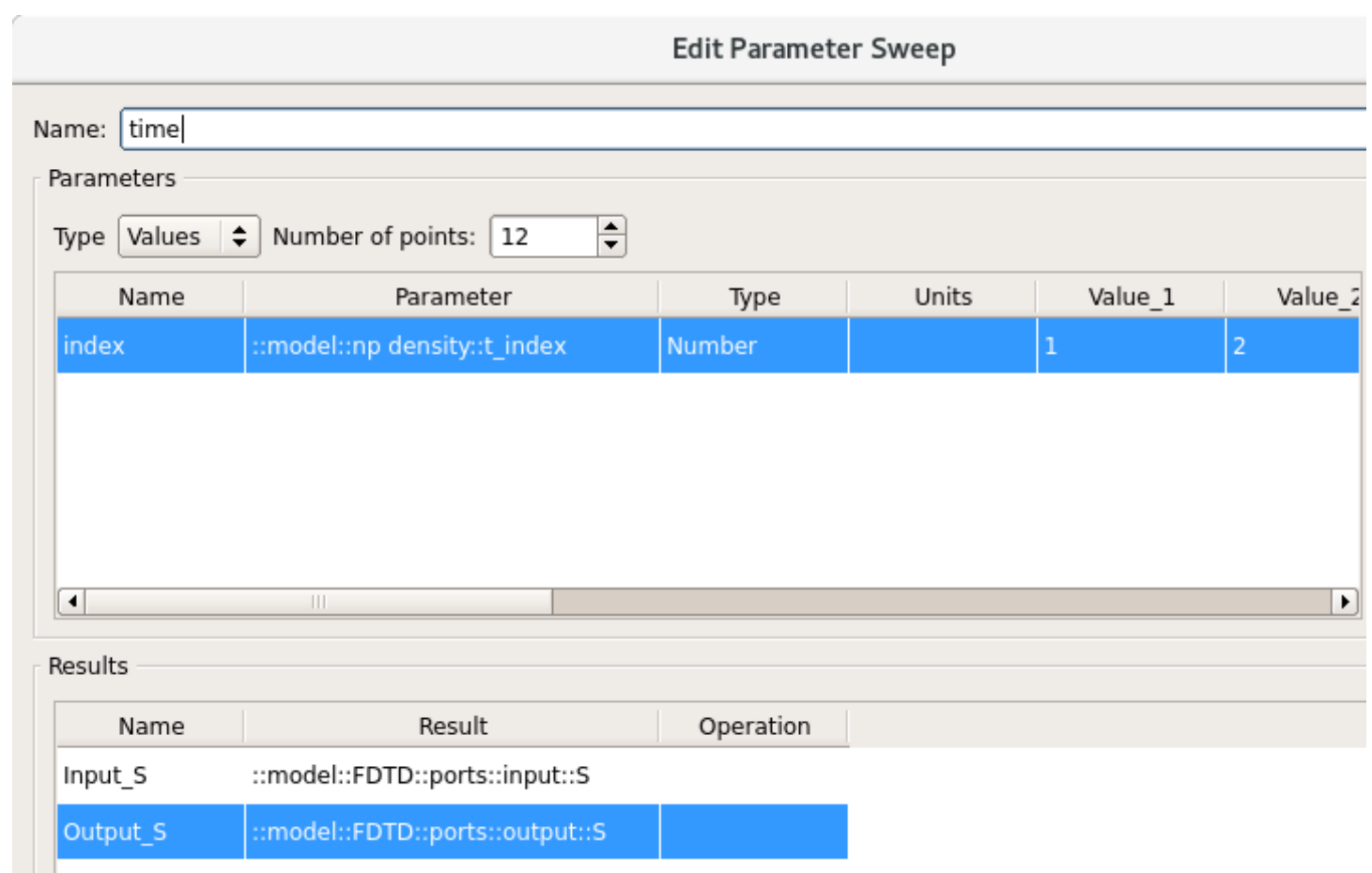

Figure 43

This parameter sweep adjusts the value of the time index of the np density charge profile to perform a series of simulations that captures the device's s-parameters as they shift over time due to the changing charge profile. Here the sweep has been setup such that the user may specify the values of time index to be simulated. This parameter sweep outputs the s-parameters as a function of the time index

By choosing the type of sweep to be "Values" (as in Figure 43), the user may specify which points in time of the charge profile will be simulated. Not every time point of the charge profile will be simulated as each one of these simulations is very costly of computational resources, and each time point chosen results in one simulation. So, a few key points along the transient may be selected, preferably several at the very beginning of the transient to capture the detail of the peak, and then a few spread out along the tail of the transient to observe the settling behavior. The number of simulations run in this example sweep is 12 . The parameter sweep must be set to store 
the s-parameters computed by the input and output ports so that the array of s-parameters may be stored as the parameter sweep results.

\section{Simulation Execution and Result Processing}

Once the parameter sweep has been appropriately setup, the sweep may be executed. This will result in several simulations running consecutively (one for each np density time index specified in the parameter sweep). This will result in two sets of results to be stored in the parameter sweep object once it is complete (one for each port). The s-parameters obtained from the output port in this example represent the forward gain (the gain from the input port to the output port) of the waveguide. The s-parameters obtained from the input port represent the reflection coefficient, which is the amount of light that gets reflected back to the input.

To convert the s-parameters to the transient transmission loss and phase shift, the sparameters in the output port are first normalized with respect to the s-parameters at time $t=0$. Since no charges have been induced into the device at that time, this normalizes the transmission and phase shift with respect to an unperturbed device. The transmission losses are then obtained by

$$
T_{\text {loss }}=1-\sqrt{r e(s(t))^{2}+i m(s(t))^{2}}
$$

and phase shift by

$$
\phi_{\text {shift }}=\tan ^{-1} \frac{i m(s(t))}{\operatorname{re}(s(t))}
$$

where $s(t)$ is the time-dependent complex-valued set of s-parameters. 


\section{Chapter Conclusions}

The simulation methodology developed in this work has been described in detail by walking through an example simulation of a slab waveguide with cross-sectional dimensions of $220 \mathrm{~nm}$ by $700 \mathrm{~nm}$ (Figure 10) in the Lumerical Tool Suite [27]. This methodology contains three stages. In the first stage, the effect of a heavy ion striking the device is emulated in Lumerical FDTD by injected a laser source with a $500 \mathrm{~nm}$ wavelength normally into the top of the waveguide. The power absorption is computed by FDTD and imported into Lumerical DEVICE for the second stage, where the generation, diffusion, and recombination of the free carriers are simulated. For the third stage, the charge profile information computed by the second stage is imported into FDTD to compute charge induced shifts in refractive index via the Soref and Bennett charge-to-index conversion model. A simulation sweep is then setup that allows the transient transmission loss and phase shift due to the SEE to be extracted. These transients characterize the effect of the ion strike on the device and may be repeated for various LET levels, device dimensions, etc. to further understand the response of the device to ionizing radiation. 


\section{CHAPTER IV}

\section{RESULTS \& DISCUSSION}

Transient s-parameters for the waveguide illustrated in Figure 10 have been computed through this simulation methodology for three levels of LET: $0.381 \mathrm{MeV}-\mathrm{cm}^{2} / \mathrm{mg}, 8.78 \mathrm{MeV}-$ $\mathrm{cm}^{2} / \mathrm{mg}$, and $40 \mathrm{MeV}-\mathrm{cm}^{2} / \mathrm{mg}$. The s-parameters have been converted to transmission loss and phase shift via Equations 3.2 and 3.3 and compiled into Figure 44 to compare the differing the transmissive properties of the waveguide overtime for ion strikes of various LETs. Note that Figure 44 only shows the beginning of the captured transients up to $250 \mathrm{ps.} \mathrm{This} \mathrm{is} \mathrm{because} \mathrm{only}$ a relatively short length of waveguide $(\sim 50 \mu \mathrm{m})$ was simulated. For the short waveguide, the charges generated in DEVICE would eventually diffuse to the point of filling up the entire waveguide with a uniform charge distribution and no means for the charge to escape. The full pulse width has not been captured in these transients due to technical issues in attempts at repeating these computations for longer $(\sim 1 \mathrm{~mm})$ lengths of waveguide. Further work will be required to address these technical issues. However, the results shown here in Figure 44 do show a characterization of the waveform peaks.

The computed transient curves of transmission loss and phase shift in Figure 44 represent the photonic equivalent of the current transients induced in electronic circuits by ion strikes like the one in Figure 4. Figure 44 shows the effects of particles of different LET on the waveguide's transmissive properties over time. These transient curves provide a basic characterization of the overall effect of the ion strike on a signal passing through the device. It is possible that these 
transients could be used as a means of developing compact models for the analysis of the effect of ion strikes on entire photonic circuits instead of just individual devices.
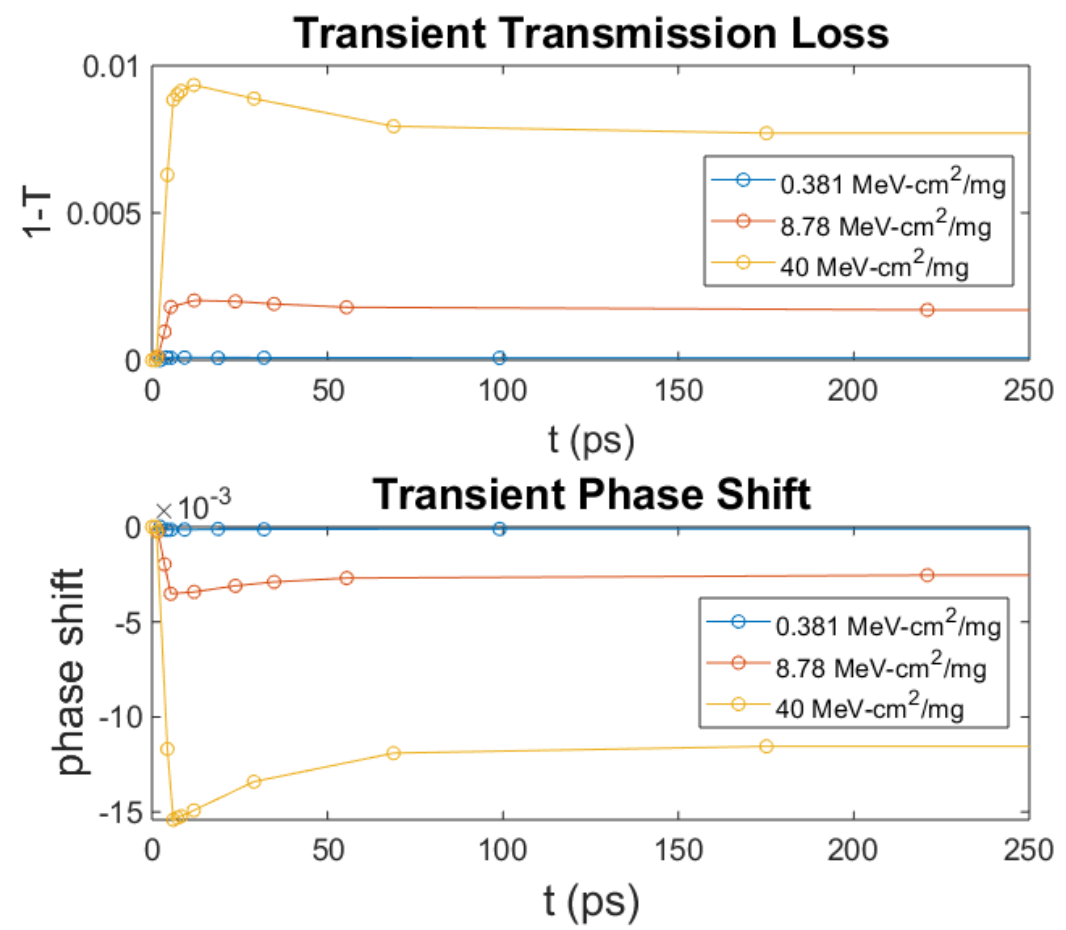

Figure 44

The transient transmission characteristics of the waveguide in Figure 10 for three LET values computed via the simulation methodology presented in this work. As LET increases, the intensity of the transients becomes more severe, reaching as high as $1 \%$ transmission loss and 0.015 radians for an LET of $40 \mathrm{MeV}-\mathrm{cm}^{2} / \mathrm{mg}$

Figure 45 shows the peak values of the transients in Figure 44 plotted versus LET. This appears to show a linear relationship between the peak induced transmission loss and the LET, and the phase shift and LET. Transients induced by the ion strike become more intense as LET increases with the peak transmission loss reaching almost $1 \%$ and the peak phase shift reaching about -0.015 radians for an LET of $40 \mathrm{MeV}-\mathrm{cm}^{2} / \mathrm{mg}$. From these results it seems that, at least for a simple straight waveguide structure and for the limited set of LETs studied here, the transmission 
loss is the dominantly affected property that could potentially lead to information loss in a photonics system. This is consistent with the findings in Goley et. al. [2].
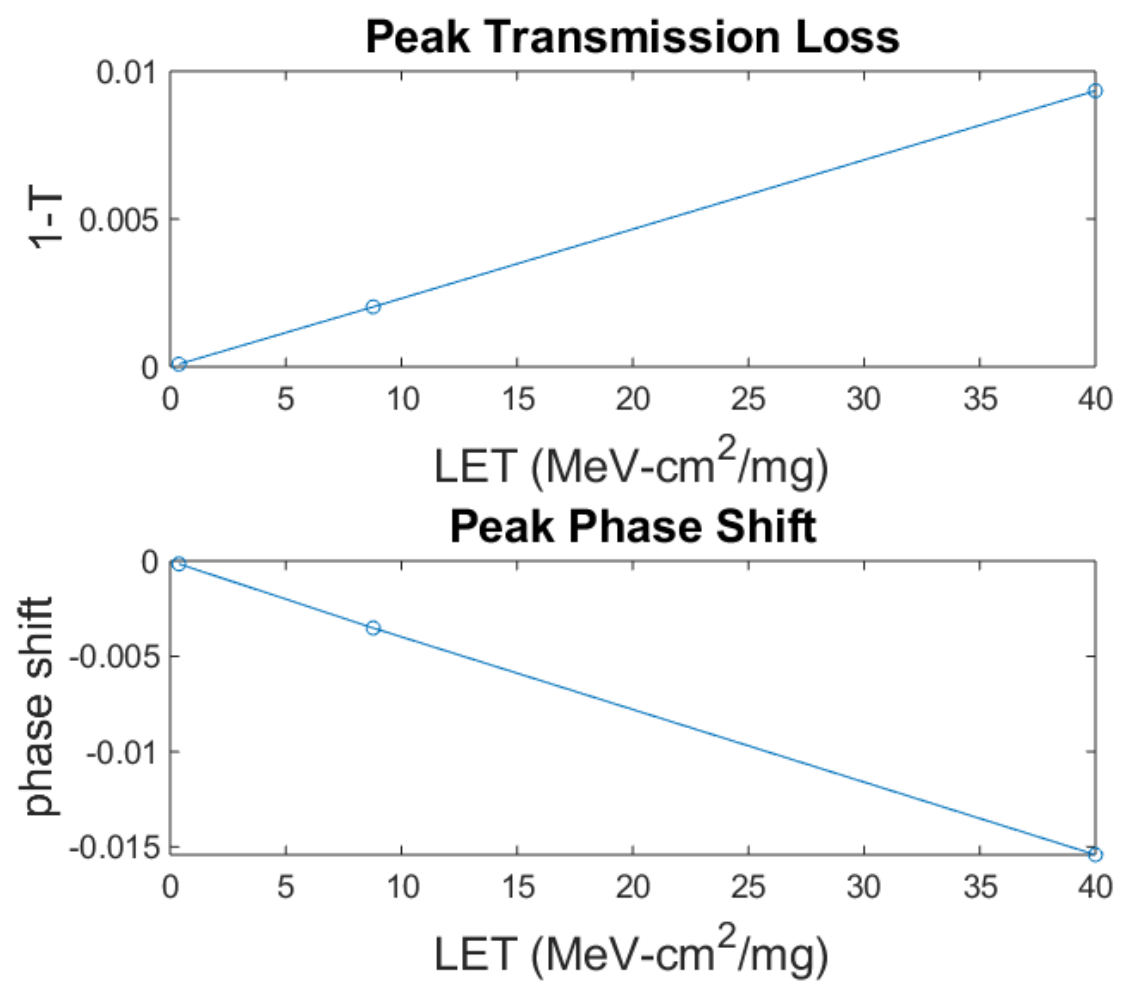

Figure 45

The peak values of transmission loss and phase shift versus LET. This graph appears to show a somewhat linear relationship between LET, peak transmission loss, and peak phase shift in the waveguide

One use of this methodology may be to aide in the discovery of techniques for hardening photonic devices to the effects of ion strikes. Although not analyzed in this work, one potential method of hardening waveguides to radiation may be to design a rib waveguide doped as a diode junction with grounded electrical contacts on each side. Such a device would be equivalent to a photonic modulator like the one in Figure 46 [4] with the two driving electrical contacts grounded. 
The electrical contacts would provide an electrical outlet for the charges generated by the ion strike and the diode junction doping provides an electric field that aides in sweeping the generated charges out of the waveguide. Such a device may also be subject to the TID radiation effects of optical modulators described in [4] where the ability for the junction to sweep charges out of the waveguide degrades as holes build up in the oxides around the modulator. Studying the effect of adding the diode junction and electrical contacts on the time constant of transients like the ones in Figure 44 may be the focus of future research, however, this work has focused primarily on fully passive waveguides to understand the baseline effect of ion strikes on these devices.

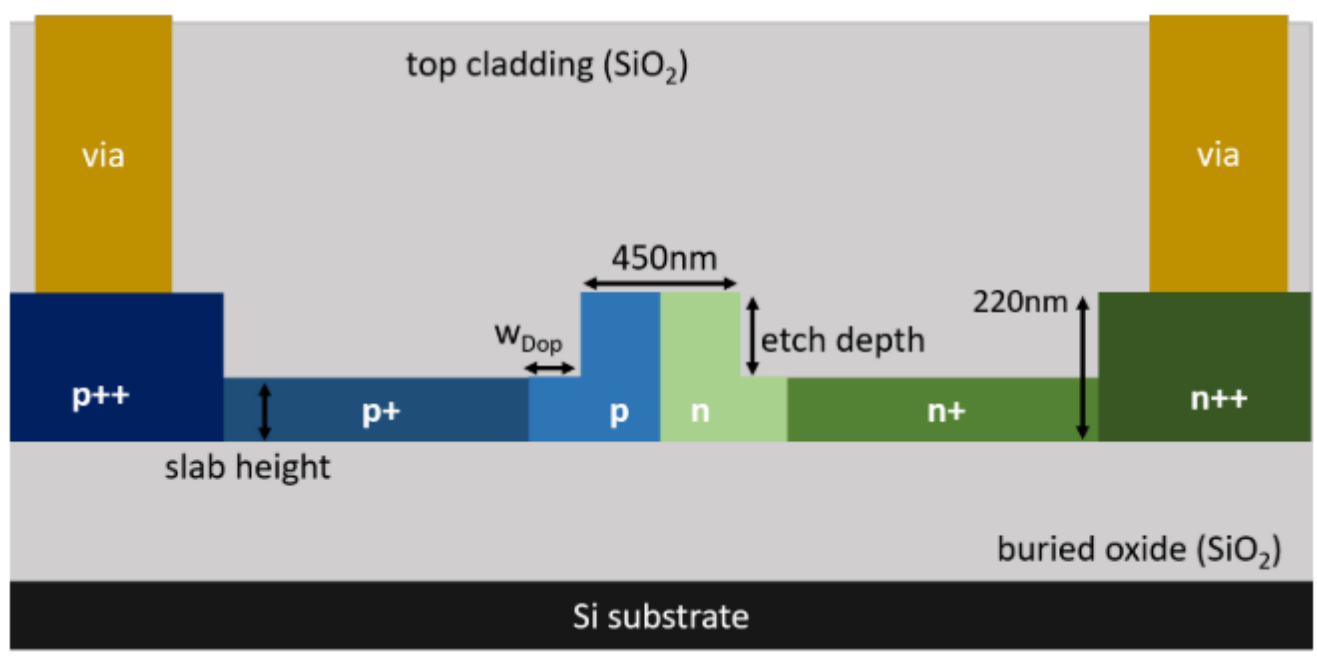

Figure 46

An example of a silicon photonic modulator. A similar structure may be useful for draining single event induced charges out of a waveguide and therefore reducing the time constant of the effect induced by these charges. [4] 


\section{CHAPTER V}

\section{CONCLUSIONS}

This work has presented a simulation methodology for analyzing the behavior of SETs in PICs and computing shifts in the transmissive properties of a photonic device as they shift over time in response to an ion strike. This methodology shows promise as a utility in characterizing the response of devices to various forms of ionizing radiation as well as discovering methods for improving the radiation hardness of photonic devices. Further work to make this methodology more automated may allow it to become more user-friendly and reduce the learning curve that is required to run these simulations.

This work has used this methodology to generate a set of transient curves for various LETs in a waveguide, showing a proportionality between transmission loss and LET as well as between phase shift and LET. Further work to validate the accuracy of this methodology, including comparison to the physical experimentation of devices under laser or radiation source, will allow this methodology to be tuned if any discrepancies are discovered. 


\section{REFERENCES}

[1] S. S. E. Nasr-Storey, F. Boeuf, C. Baudot, S. Detraz, J. M. Fedeli, D. Marris-Morini, L. Olantera, G. Pezzullo, C. Sigaud, C. Soos, J. Troska, F. Vasey, L. Vivien, M. Zeiler and M. Ziebell, "Modeling TID Effects in Mach-Zehnder Interferometer Silicon Modulator for HL-LHC Data Transmission Applications," IEEE Trans. Nucl. Sci., vol. 62, no. 6, pp. 2971-2978, 2015.

[2] P. S. Goley, Z. E. Fleetwood and J. D. Cressler, "Potential Limitations on Integrated Silicon Photonic Waveguides Operating in a Heavy Ion Environment," IEEE Trans. Nucl. Sci., vol. 65, no. 1, pp. 141-148, 2018.

[3] S. Bhandaru, S. Hu, D. M. Fleetwood and S. M. Weiss, "Total ionizing dose effects on silicon ring resonators," IEEE Trans. Nucl. Sci., vol. 62, no. 1, pp. 323-328, 2015.

[4] M. Zeiler, S. S. E. Nasr-Storey, S. Detraz, A. Kraxner, L. Olantera, C. Scarcella, C. Sigaud, C. Soos, J. Troska and F. Vasey, "Radiation Damage in Silicon Photonic MachZehnder Modulators and Photodiodes," IEEE Trans. Nucl. Sci., vol. 64, no. 11, pp. 2794-2801, 2017.

[5] M. Zeiler, S. Detraz, L. Olantera, C. Sigaud, C. Soos, J. Troska and F. Vasey, "Comparison of the radiation hardness of silicon Mach-Zehnder modulators for different DC bias voltages," in IEEE Nuclear Science Symposium, Strasbourg, France, 2016.

[6] S. Alt and J. Pellish, "Photonic Integrated Circuit (PIC) Device Structures: Background, Fabrication Ecosystem, Relevance to Space Systems Applications, and Discussion of Related Radiation Effects," 15 April 2016. [Online]. Available:

https://nepp.nasa.gov/files/26909/2016-561-Alt-Final-NEPPweb-IP-BOK-TN34661.pdf. [Accessed 26 April 2019].

[7] H. J. Barnaby, "Total-Ionizing-Dose Effects in Modern CMOS Technologies," IEEE Trans. Nucl. Sci., vol. 53, no. 6, pp. 3103-3121, 2006.

[8] L. W. Massengill, B. L. Bhuva, W. T. Holman, M. L. Alles and T. D. Loveless, "Technology Scaling and Soft Error Reliability," in 2012 IEEE International Reliability Physics Symposium (IRPS), Anaheim, Anaheim, CA, 2012. 
[9] T. Pinguet et. al., "Silicon photonics multiore transceivers," in IEEE Photonics Society Summer Topical Meeting Series, Seattle, WA, 2012.

[10] R. B. Wehrspohn et. al., "Photonic bandgap waveguide structures," in Conferenct on Lasers and Electro-Optics Europe, Munich, Germany, 2003.

[11] D. T. Pierce and W. E. Spicer, "Electronic Structure of Amorphous Si from Photoemission and Optical Studies," Phys. Rev. B. 5, vol. 5, no. 8, pp. 3017-3029, 1972.

[12] C. Z. Tan, "Determination of refractive index of silica glass for infrared wavelengths by IR spectroscopy," J. Non-Cryst. Solids, vol. 223, no. 1-2, pp. 158-163, 1998.

[13] W. A. Gambling, D. N. Payne and H. Matsumura, "Mode excitation in a multimode optical-fibre waveguide," Electronics Letters, vol. 9, no. 18, pp. 412-414, 1973.

[14] H. Ikeuchi, S. Matsumoto, T. Kawai and I. Ohta, "A novel TE10-TE20 mode transducer utilizing vertical cross-excitation," in IEEE MTT-S International Microwave Symposium, Anaheim, CA, 2010.

[15] L. Chrotowski and M. Hochberg, Silicon Photonics Design: From Devices to Systems, Cambridge University Press, 2015.

[16] A. H. Johnston, "Space Radiation Effects and Reliability Considerations for Micro- and Optoelectronic Devices," IEEE Transactions on Device and Materials Reliability, vol. 10, no. 4, pp. 449-459, 2010.

[17] K. Endo, "The Radiation Environment," Nikkei Science, Inc. of Japan.

[18] L. W. Massengill, "SEU Modeling and Prediction Techniques," IEEE NSREC Short Course, vol. 3, 1993.

[19] S. P. Buchner, F. Miller, V. Pouget and D. P. McMorrow, "Pulsed-Laser Testing for Single-Event Effects Investigations," IEEE Trans. Nucl. Sci., vol. 60, no. 3, pp. 18521875, 2013.

[20] J. M. Hales, A. Khachatrian, S. Buchner, N. J.-H. Roche, J. Warner, V. Ferlet-Cavrois and D. McMorrow, "Using Two-Photon Absorption Pulsed-Laser Excitation to Simulate Radiation Effects in Microelectronics," in 2018 Conference on Lasers and ElectroOptics (CLEO), San Jose, CA, 2018. 
[21] M. H. Jones and S. H. Jones, "Optical Properties of Silicon," [Online]. Available: https://www.univie.ac.at/photovoltaik/vorlesung/ss2012/unit4/optical_properties_silicon. pdf.

[22] A. D. Bristow, N. Rotenburg and H. M. van Driel, "Two-photon absorption and Kerr coefficients fo silicon for 850-2200 nm," Applied Physics Letters, vol. 90, no. 19, 2007.

[23] V. R. Almeida, C. A. Barrios, R. R. Panepucci and M. Lipson, "All-optical control of light on a silicon chip," Nature, vol. 431, pp. 1081-1084, 2004.

[24] K. A. Hallman, R. E. Marvel, J. D. Ryckman, S. M. Weiss and R. F. Haglund, "Ultracompact silicon photonic devices reconfigured by an optically induced semiconductorto-metal transition," Optics Express, vol. 21, no. 9, pp. 10753-10763, 2013.

[25] R. Soref and B. Bennett, "Electrooptical effects in silicon," IEEE Journal of Quantum Electronics, vol. 23, no. 1, pp. 123-129, 1987.

[26] "Lumerical FDTD Solver," [Online]. Available: https://kb.lumerical.com/solvers_finite_difference_time_domain.html. [Accessed 16 2019].

[27] [Online]. Available: www.lumerical.com. [Accessed 16 June 2019].

[28] [Online]. Available: www.synopsys.com. [Accessed 16 June 2019]. 


\section{APPENDIX A}

\section{SCRIPTS}




\section{Charge Profile Integration and LET Estimation MATLAB Script}

This MATLAB script integrates the total charge in Lumerical DEVICE charge profile and plots the total number of electrons over time. This script assumes that the number of electrons and holes remain approximately the same over time and therefore assumes the maximum amount of electrons is equal to the total number of EHPs. The maximum number of electrons is used to estimate the LET of the ion strike.

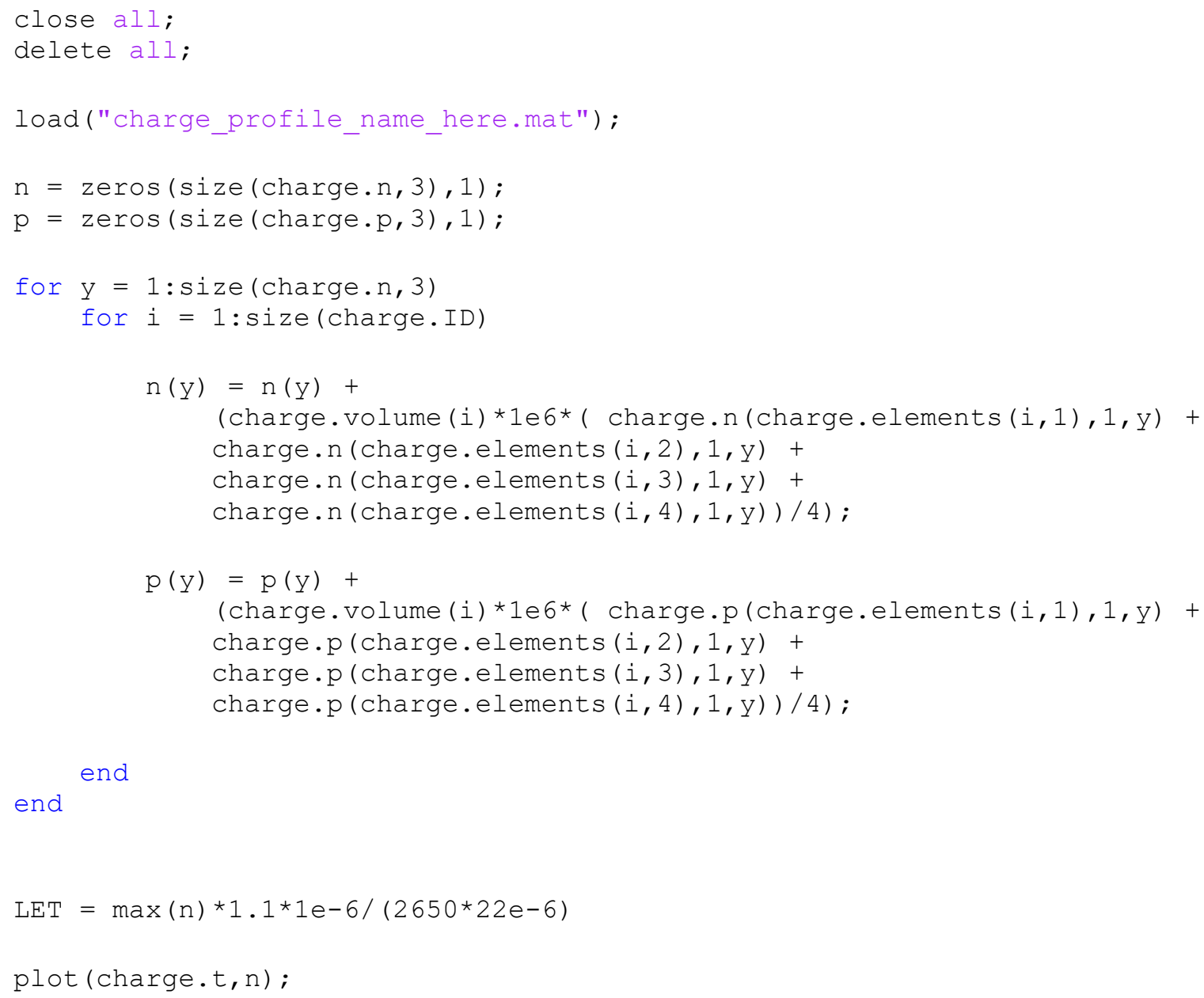




\section{VITA}

Ryan Boggs was born June 10, 1994 in Havre de Grace, Maryland to parents Mary and Randy Boggs. He is the second of two children, an older sister. Before graduating Bradley Central High School in Cleveland, Tennessee, he gained a passionate interest in electronics. After graduating high school in 2012, he attended Cleveland State Community College. In 2014 he transferred to the University of Tennessee at Chattanooga to finish his Bachelor's of Science in Electrical Engineering which he completed in May 2017. In the summer of 2017 he became a graduate research assistant at UTC under Dr. Daniel Loveless while working on his master's degree. In the fall of 2018 he got the opportunity to intern at the NASA Goddard Space Flight Center in Greenbelt, Maryland for their instrument electronics development branch and returned to finish his master's degree in spring 2019. Ryan hopes to return to NASA to continue working in electronics design for space systems. 